JOURNAL OF THE

AMERICAN MATHEMATICAL SOCIETY

Volume 11, Number 1, January 1998, Pages 189-228

S 0894-0347(98)00252-5

\title{
ON HITCHIN'S CONNECTION
}

\author{
BERT VAN GEEMEN AND AISE JOHAN DE JONG
}

\section{INTRODUCTION}

1.1. The aim of this paper is to give an explicit expression for Hitchin's connection in the case of rank 2 bundles with trivial determinant over curves of genus 2 .

We sketch the general situation. Let $\pi: \mathcal{C} \rightarrow S$ be a family of projective smooth curves. Let $p: \mathcal{M} \rightarrow S$ be the family of moduli spaces of ( $S$-equivalence classes of) rank $r$ bundles with trivial determinant on $\mathcal{C}$ over $S$. On $\mathcal{M}$ we have a naturally defined determinant line bundle $\mathcal{L}$. The vector bundles $p_{*}\left(\mathcal{L}^{\otimes k}\right)$ on $S$ have a natural (projective) flat connection (such a connection also exists for other structure groups besides $S L(r))$.

This connection first appeared in Quantum Field Theory (conformal blocks) and was subsequently studied by various mathematicians (see [Hi] and [BM] and the references given there). We follow the approach of Hitchin [Hi] who showed that this connection can be constructed along the lines of Welters' theory of deformations [W].

In case the curves have genus 2 and the rank $r=2$, the family of moduli spaces $p: \mathcal{M} \rightarrow S$ is isomorphic (over $S$ ) to a $\mathbf{P}^{3}$-bundle

$$
p: \mathcal{M} \cong \mathbf{P} \longrightarrow S \text {. }
$$

Under this isomorphism $\mathcal{L}$ corresponds to $\mathcal{O}_{\mathbf{P}}(1) \otimes p^{*} \mathcal{N}$ for some line bundle $\mathcal{N}$ on $S$. Giving a projective connection on $p_{*} \mathcal{L}^{\otimes k}$ is then the same as giving a projective connection on $p_{*} \mathcal{O}_{\mathbf{P}}(k)$

$$
\nabla^{(k)}: p_{*} \mathcal{O}_{\mathbf{P}}(k) \longrightarrow p_{*} \mathcal{O}_{\mathbf{P}}(k) \otimes \Omega_{S}^{1} .
$$

1.2. In the first part of the paper (Section 2) we give a characterization of Hitchin's connection (strictly speaking, we define a new connection which is almost characterized by Hitchin's requirements).

We first develop a bit of general theory concerning heat operators. In the situation above a heat operator is a certain type of map $D: p^{*}\left(\mathcal{T}_{S}\right) \rightarrow \operatorname{Diff}_{\mathcal{M}}^{(2)}\left(\mathcal{L}^{\otimes k}\right)$. Thus a vector field $\theta$ on $S$ gives a second order differential operator $D(\theta)$ on $\mathcal{L}^{\otimes k}$, which determines a map $D(\theta): p_{*} \mathcal{L}^{\otimes k} \rightarrow p_{*} \mathcal{L}^{\otimes k}$. These then determine a connection on $p_{*} \mathcal{L}^{\otimes k}$. The symbol of $D$ is a map $\rho_{D}: p^{*}\left(\mathcal{T}_{S}\right) \rightarrow S^{2} \mathcal{T}_{\mathcal{M} / S}$.

Let $\mathcal{M}^{s}$ be the locus of stable bundles in $\mathcal{M}$. The codimension of the non-stable locus in $\mathcal{M}$ is at least two if $g \geq 3$ so that $\left.p_{*} \mathcal{L}^{\otimes k}\right|_{\mathcal{M}^{s}}=p_{*} \mathcal{L}^{\otimes k}$ in this case. Hitchin

Received by the editors January 16, 1997 and, in revised form, September 4, 1997.

1991 Mathematics Subject Classification. Primary 14H60, 53C05; Secondary 20F36, 32G15, $14 \mathrm{D} 20$.

Key words and phrases. Hitchin's connection, moduli of vector bundles, heat equations, heat operators.

(C) 1998 American Mathematical Society 
showed for $g \geq 3$ that if a heat operator $D$ on $\mathcal{L}^{\otimes k}$ over $\mathcal{M}^{s}$ determines the desired connection on $p_{*} \mathcal{L}^{\otimes k}$, then its symbol map:

$$
\rho_{D}^{s}: p^{*}\left(\mathcal{T}_{S}\right) \longrightarrow S^{2} \mathcal{T}_{\mathcal{M}^{s} / S},
$$

must be equal to a map $1 /(k+2) \rho_{\text {Hitchin }}^{s}$. The map $\rho_{\text {Hitchin }}^{s}$ is determined by the deformation theory of a typical fibre $C$ and of the stable bundles on $C$. He also proved that $\rho_{\text {Hitchin }}^{s}$ extends to $\rho_{\text {Hitchin }}: p^{*}\left(\mathcal{T}_{S}\right) \rightarrow S^{2} \mathcal{T}_{\mathcal{M} / S}$ for bundles of rank $r$ on curves of genus $g$, except maybe for the case $r=2, g=2(!)$.

We show, using simple bundles, that $\rho_{\text {Hitchin }}^{s}$ also extends to $\rho_{\text {Hitchin }}$ in the case $g=2$ (Theorem 2.6.1). From a study of heat operators and their symbols we conclude that we can find a (projective) heat operator $D$ with the right symbol over all of $\mathcal{M}$ for $g=2$ (Corollary 2.6.3). Such a $D$ need not be unique however. To pin it down we use the action of the two-torsion group scheme $\mathcal{H}:=\operatorname{Pic}_{\mathcal{C} / S}^{0}[2]$ on $\mathcal{M}$ (tensoring a rank two bundle by a line bundle of order two doesn't change the determinant). Then $\mathcal{H}$ acts (projectively) on $\mathcal{L}^{\otimes k}$; this action can be linearized to the action of a central extension $\mathcal{G}$ of $\mathcal{H}$ on $\mathcal{L}^{\otimes k}$ :

$$
1 \longrightarrow \mathbf{G}_{m} \longrightarrow \mathcal{G} \longrightarrow \mathcal{H} \longrightarrow 0
$$

We show that there is a unique (projective) heat operator

$$
\mathbf{D}_{k}: p^{*}\left(\mathcal{T}_{S}\right) \longrightarrow \operatorname{Diff}_{\mathcal{M}}^{(2)}\left(\mathcal{L}^{\otimes k}\right) \quad \text { with } \quad \rho_{\mathbf{D}_{k}}=\frac{1}{k+2} \rho_{\text {Hitchin }}
$$

which commutes with the action of $\mathcal{G}$ (Proposition 2.6.4). The (projective) connection determined by $\mathbf{D}_{k}$ is called Hitchin's connection (cf. 2.6.6).

1.3. To be able to compute $\mathbf{D}_{k}$ (and to establish some of the results mentioned above), we use a map $f: \mathrm{Pic}_{\mathcal{C} / S}^{0} \rightarrow \mathcal{M}$ over $S$ whose image is the family of Kummer surfaces $\mathcal{K} \rightarrow S$. We show that the pullback of $\mathbf{D}_{k}$ along $f$ determines the connection on $q_{*} f^{*} \mathcal{L}^{\otimes k}$ given by the heat equations on (abelian) theta functions. (The connection defined by $\mathbf{D}_{k}$ on $p_{*} \mathcal{L}^{\otimes k}$ does not pullback to the 'abelian' connection however.)

We show that $\mathbf{D}_{k}$ is characterized by this property. Moreover, we express this property in terms of the (local) equation defining $\mathcal{K} \subset \mathcal{M}(2.6 .7(E q)$.$) .$

1.4. To write down a completely explicit connection (on $p_{*} \mathcal{O}_{\mathbf{P}}(k)$ ) we must now choose a certain $S$ and a family of curves $\mathcal{C}$ over $S$. We take $S=\mathcal{P}$, the configuration space of $2 g+2$ points in $\mathbf{C}^{2 g+2}$ and take the obvious family of hyperelliptic curves over it:

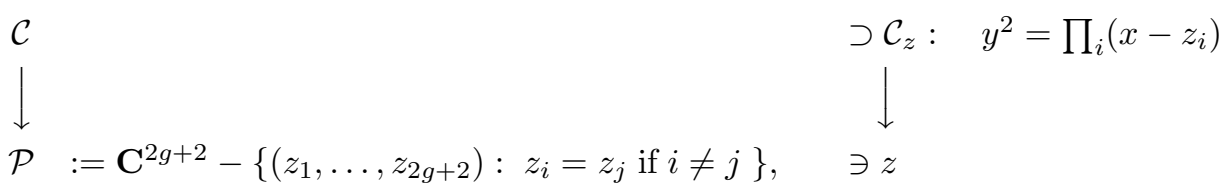

In the case $g=2$ this family dominates the moduli space of genus 2 curves (the induced morphism of $\mathcal{P}$ to the stack of genus two curves is smooth and surjective). We will define connections on bundles over $\mathcal{P}$ for any $g$. In the case $g=2$ this connection is basically the Hitchin connection (see Theorem 4.4.2). It seems possible that for general $g$ the connection is related to the Hitchin connection for the family of hyperelliptic curves above. 
For $g=2$ we can see which bundle to use: The pullback of the bundle $p: \mathbf{P} \rightarrow \mathcal{P}$ along an unramified $2^{4}: 1$-Galois cover $\tilde{\mathcal{P}} \rightarrow \mathcal{P}$ with group $H=(\mathbf{Z} / 2 \mathbf{Z})^{4}$ is trivial. The bundle $\mathbf{P}$ can thus be obtained as a quotient of $\tilde{\mathcal{P}} \times \mathbf{P}^{3}$ by $H$ (4.4.1). Therefore, locally (in the complex or the étale topology), the vector bundles $p_{*} \mathcal{O}_{\mathbf{P}}(k)$ and the trivial bundle $S_{k} \otimes \mathcal{O}_{\mathcal{P}}$ are canonically isomorphic, with $S_{k}=H^{0}\left(\mathbf{P}^{3}, \mathcal{O}(k)\right)$, the vector space of homogeneous polynomials of degree $k$ in 4 variables. Thus it suffices to write down the connection on the trivial bundle which corresponds to Hitchin's connection on $p_{*} \mathcal{O}_{\mathbf{P}}(k)$.

This connection on $S_{k} \otimes \mathcal{O}_{\mathcal{P}}$ (for general $g$ ) is given by

$$
\nabla: S_{k} \otimes \mathcal{O}_{\mathcal{P}} \longrightarrow S_{k} \otimes \Omega_{\mathcal{P}}, \quad \nabla(w \otimes f)=w \otimes \mathrm{d} f-\frac{1}{k+2} \sum_{i \neq j} M_{i j}(w) \otimes \frac{f \mathrm{~d} z_{i}}{z_{i}-z_{j}},
$$

for certain endomorphisms $M_{i j} \in \operatorname{End}\left(S_{k}\right)$. We give two descriptions of the endomorphisms $M_{i j}$. One is based on the Lie algebra $s o(2 g+2)$ and its (half) spin representations. We will in fact identify $S_{k}=S^{k} V\left(\omega_{g+1}\right)$ where $V\left(\omega_{g+1}\right)$ is a half spin representation of $s o(6)$.

The other description uses the Heisenberg group action and we sketch that description here. The family $\mathcal{C}$ has $2 g+2$ sections which are the Weierstrass points on each fibre:

$$
P_{i}: \mathcal{P} \longrightarrow \mathcal{C}, \quad z \longmapsto(x, y)=\left(z_{i}, 0\right) .
$$

Each $P_{i}-P_{j}$ is a section in $\operatorname{Pic}_{\mathcal{C} / S}^{0}[2](S) \cong(\mathbf{Z} / 2 \mathbf{Z})^{2 g}$. There is a central extension $G$ of this group which acts on the $S_{k}$ 's (the group scheme $\mathcal{G}$ is a twist of the constant group scheme $G$, these group schemes are isomorphic over $\tilde{P})$. Let $\pm U_{i j} \in \operatorname{End}\left(S_{1}\right)$ be the endomorphisms induced by $P_{i}-P_{j}$ with the property that $U_{i j}^{2}$ is the identity. Since $\operatorname{End}\left(S_{1}\right)=S_{1} \otimes S_{1}^{*}$ and $S_{1}^{*}$ may be identified with the derivations on $S:=$ $\bigoplus S_{k}$ and the composition $U_{i j} U_{i j}$ may be considered as a second order differential operator which acts on any $S_{k}$, the symbol (that is, the degree two part) of this operator is, up to a factor $-1 / 16$, the desired $M_{i j}$ (which, in this sense, does not depend on $k$ ).

The idea for trying these $\Omega_{i j}$ 's comes from [vGP]. There the Hitchin map, a Hamiltonian system on the cotangent bundle $T^{*} \mathcal{M}^{s}$, is studied. The Hitchin map is basically the symbol of the heat operator $\mathcal{D}_{k}(\mathrm{cf}$. [Hi], §4). Among the results of [vGP] is an explicit description of the Hitchin map for $g=2$ curves (obtained from line geometry in $\mathbf{P}^{3}$ ) and implicit in that description is the one for the $M_{i j}$ given here.

1.5. Now that the connection is determined it is interesting to consider its monodromy representation. In the case of rank two bundles, this has been considered by Kohno [K2]. We verify that our connection has the same local monodromy as Kohno's representation in Subsection 4.5. Here local monodromy means "at $\infty$ ", e.g., we determine the eigenvalues of the monodromy operator of a small loop around the divisor $z_{1}=z_{2}$ in $\mathcal{P}$. Kohno (and the physicists) use (locally on the base it seems) the Knizhnik-Zamolodzhikov equations (which define a flat connection over $\mathcal{P}$ using the Lie algebra $s l(2)$ [Ka], Chapter XIX). It is not clear to us how the KZ-equations are related to our connection (which is based on $s o(2 g+2)$ ). We do not know which representations of the braid group one obtains from our connections. 


\section{Characterizing Hitchin's CONnECtion}

2.1. Introduction. We recall some deformation theory as described by Welters $[\mathrm{W}]$ and relate it to connections. Then we introduce heat operators and show how they define (projective) connections on certain bundles. Next we give a criterion for the existence of a heat equation with a given symbol and in Section 2.4 we recall the definition of the symbol of Hitchin's connection. In Section 2.5 we combine these results and give criteria (in 2.6.7 and 2.6.6) which determine Hitchin's connection.

\subsection{Deformation theory.}

2.2.1. Deformations. Let $X$ be a smooth projective variety over $\mathbf{C}$ with tangent bundle $T_{X}$. Let $L$ be an invertible $\mathcal{O}_{X}$-module on $X$ and let $s \in H^{0}(X, L)$. We are going to classify first order deformations of $X$, of the pair $(X, L)$ and of the triple $(X, L, s)$. See $[\mathrm{W}]$ for proofs.

Isomorphism classes of first order deformations $X_{\epsilon}$ of $X$ (over $\operatorname{Spec} \mathbf{C}[\epsilon] /\left(\epsilon^{2}\right)$ ) are classified by $H^{1}\left(X, T_{X}\right)$. We write $\left[X_{\epsilon}\right]$ for the class in $H^{1}\left(X, T_{X}\right)$ of the deformation $X_{\epsilon}$; similar notation will be used throughout.

The first order deformations $\left(X_{\epsilon}, L_{\epsilon}\right)$ of the pair $(X, L)$ are classified by $H^{1}\left(X, \operatorname{Diff}_{X}^{(1)}(L)\right)$. Here $\operatorname{Diff}_{X}^{(1)}(L)$ is the sheaf of first order differential operators on $L$. This sheaf sits in an exact sequence:

$$
0 \longrightarrow \mathcal{O}_{X} \longrightarrow \operatorname{Diff}_{X}^{(1)}(L) \stackrel{\sigma}{\longrightarrow} T_{X} \longrightarrow 0
$$

where the map $\sigma: \operatorname{Diff}_{X}^{(1)}(L) \rightarrow T_{X}$ gives the symbol of the operator. This sequence gives a map $\alpha: H^{1}\left(X, \operatorname{Diff}_{X}^{(1)}(L)\right) \rightarrow H^{1}\left(X, T_{X}\right)$, which maps $\left[\left(X_{\epsilon}, L_{\epsilon}\right)\right]$ to $\left[X_{\epsilon}\right]$.

Finally, we consider first order deformations of the triple $(X, L, s)$. The evaluation map $d^{1} s: \operatorname{Diff}_{X}^{(1)}(L) \rightarrow L, D \mapsto D s$ gives a complex:

$$
0 \longrightarrow \operatorname{Diff}_{X}^{(1)}(L) \stackrel{d^{1} s}{\longrightarrow} L \longrightarrow 0 .
$$

Let $\mathbf{H}^{1}\left(d^{1} s\right)$ be the first hypercohomology group of this complex. This is the space classifying the isomorphism classes of deformations $\left(X_{\epsilon}, L_{\epsilon}, s_{\epsilon}\right)$ of $(X, L, s)([\mathrm{W}]$, Prop. 1.2). The spectral sequence connecting hypercohomology to cohomology gives a map $\beta: \mathbf{H}^{1}\left(d^{1} s\right) \rightarrow H^{1}\left(\operatorname{Diff}_{X}^{(1)}(L)\right)$, which maps $\left[\left(X_{\epsilon}, L_{\epsilon}, s_{\epsilon}\right)\right]$ to $\left[\left(X_{\epsilon}, L_{\epsilon}\right)\right]$.

Any element of $\mathbf{H}^{1}\left(d^{1} s\right)$ may be represented by a Čech cocycle. Let us choose an affine open covering $\mathcal{U}: X=\bigcup U_{i}$ and write $U_{i j}=U_{i} \cap U_{j}$. A Čech cocycle is given by a pair $\left(\left\{t_{i}\right\},\left\{D_{i j}\right\}\right)$ satisfying the relations:

$$
t_{i}-t_{j}=D_{i j}(s), \quad D_{j k}-D_{i k}+D_{i j}=0 \quad\left(t_{i} \in L\left(U_{i}\right), D_{i j} \in \operatorname{Diff}_{X}^{(1)}(L)\left(U_{i j}\right)\right) .
$$

The maps $\beta$ and $\alpha \circ \beta$ correspond to forgetting first $s_{\epsilon}$ and then $L_{\epsilon}$. They can be described as follows. The cocycle $\left(\left\{t_{i}\right\},\left\{D_{i j}\right\}\right)$ is mapped to the 1-cocycle $\left\{D_{i j}\right\}$ in the sheaf $\operatorname{Diff}_{X}^{(1)}(L)$ under $\beta$. This is then mapped to the 1-cocycle $\left\{\sigma^{(1)}\left(D_{i j}\right)\right\}$ in the sheaf $T_{X}$.

Let us make explicit a deformation $\left(X_{\epsilon}, L_{\epsilon}, s_{\epsilon}\right)$ associated to $\left(\left\{t_{i}\right\},\left\{D_{i j}\right\}\right)$. Write $U_{i}=\operatorname{Spec}\left(A_{i}\right)$ and $U_{i j}=\operatorname{Spec}\left(A_{i j}\right)$. Let $M_{i}=L\left(U_{i}\right)$ and $M_{i j}=L\left(U_{i j}\right)$. The section $s$ gives elements $s_{i} \in M_{i}$; note that $t_{i} \in M_{i}$ as well. Write $\theta_{i j}=\sigma\left(D_{i j}\right) \in$ $T_{X}\left(U_{i j}\right)$. The 1-cocycle $\left\{\theta_{i j}\right\}$ defines a scheme $X_{\epsilon}$ over $\operatorname{Spec}(\mathbf{C}[\epsilon])$ by glueing the rings $A_{i}[\epsilon]$ via the isomorphisms

$$
A_{i j}[\epsilon] \longrightarrow A_{i j}[\epsilon], \quad f+g \epsilon \longmapsto f+\left(\theta_{i j}(f)+g\right) \epsilon .
$$


Here we view $\theta_{i j}$ as a derivation. The 1-cocycle $\left\{D_{i j}\right\}$ in $\operatorname{Diff}_{X}^{(1)}(L)$ gives a deformation $L_{\epsilon}$ of $L$ by glueing the $A_{i}[\epsilon]$-modules $M_{i}[\epsilon]$ via the $A_{i j}[\epsilon]$-module isomorphisms:

$$
\phi_{i j}: M_{i j}[\epsilon] \longrightarrow M_{i j}[\epsilon], \quad m_{i j}+m_{i j}^{\prime} \epsilon \longmapsto m_{i j}+\left(D_{i j}\left(m_{i j}\right)+m_{i j}^{\prime}\right) \epsilon .
$$

Note one has $D_{i j}(f s)=f D_{i j}(s)+\theta_{i j}(f) s$ by definition of the symbol $\sigma$ and $\sigma\left(D_{i j}\right)=$ $\theta_{i j}$. The cocycle $\left(\left\{t_{i}\right\},\left\{D_{i j}\right\}\right)$ in $\mathbf{H}^{1}\left(d^{1} s\right)$ defines a deformation $s_{\epsilon} \in H^{0}\left(X_{\epsilon}, L_{\epsilon}\right)$. The section $s_{\epsilon}$ is given by the family of elements:

$$
s_{\epsilon}:=\left\{s_{\epsilon, i}\right\} \quad\left(\in M_{i}[\epsilon]\right) \quad \text { with } \quad s_{\epsilon, i}:=s_{i}+t_{i} \epsilon .
$$

The cocycle relation $t_{i}-t_{j}=D_{i j}(s)$ shows that the $s_{\epsilon, i}$ 's glue: $\phi_{i j}\left(s_{\epsilon, j}\right)=s_{\epsilon, i}$.

2.2.2. Second order operators. The following construction is useful to obtain elements in $\mathbf{H}^{1}\left(d^{1} s\right)$ ([W], (1.9)). Let Diff ${ }_{X}^{(2)}(L)$ be the sheaf of second order differential operators on $L$. The symbol gives an exact sequence which is the first row of the complex:

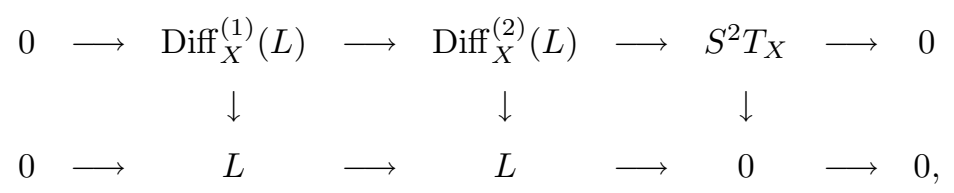

where the vertical maps are evaluation on $s(D \mapsto D s)$, thus the first column is the complex $d^{1} s$ considered before. From the exact sequence of hypercohomology one obtains a map

$$
\delta_{s}: H^{0}\left(S^{2} T_{X}\right) \longrightarrow \mathbf{H}^{1}\left(d^{1} s\right) .
$$

Let us give a description in terms of cocycles of this map. Let $\mathcal{U}=\left\{U_{i}\right\}$ be an affine open cover of $X$ as before. Let $w \in H^{0}\left(X, S^{2} T_{X}\right)$. We can find operators $D_{i}^{(2)} \in \operatorname{Diff}_{X}^{(2)}(L)\left(U_{i}\right)$ which map to $\left.w\right|_{U_{i}}$ in $S^{2} T_{X}\left(U_{i}\right)$. Then

$$
\delta_{s}(w)=\left(\left\{D_{i}^{(2)}(s)\right\},\left\{D_{i}^{(2)}-D_{j}^{(2)}\right\}\right) \quad\left(\in \mathbf{H}^{1}\left(d^{1} s\right)\right) .
$$

It is easy to verify that $\delta_{s}(w)$ is indeed a cocycle for the complex defined by $d^{1} s$. Note $w=\sigma\left(D_{i}^{(2)}\right)=\sigma\left(D_{j}^{(2)}\right)$ on $U_{i j}$, thus $D_{i}^{(2)}-D_{j}^{(2)}$ is a first order operator.

Note that $\beta\left(\delta_{s}(w)\right)=\left\{D_{i}^{(2)}-D_{j}^{(2)}\right\}\left(\in \operatorname{Diff}_{X}^{(1)}(L)\right)$ is independent of the choice of the section $s$. Therefore, given $w\left(\in H^{0}\left(X, S^{2} T_{X}\right)\right)$ we get a deformation $\left(X_{\epsilon}, L_{\epsilon}\right)$ such that any section $s \in H^{0}(X, L)$ is deformed to a section $s_{\epsilon} \in H^{0}\left(X_{\epsilon}, L_{\epsilon}\right)$.

2.2.3. Infinitesimal connections. Let $\left(X_{\epsilon}, L_{\epsilon}\right)$ be a deformation of $(X, L)$. We consider a differential operator $D$ of order at most two on $L_{\epsilon}$ which locally can be written as (notations as above, cf. 2.3.2 below)

$$
D=\left\{D_{i}\right\} \quad\left(\in H^{0}\left(X_{\epsilon}, \operatorname{Diff}_{X_{\epsilon}}^{(2)}\left(L_{\epsilon}\right)\right)\right), \quad D_{i}=\partial_{\epsilon}+R_{i}: M_{i}[\epsilon] \longrightarrow M_{i}[\epsilon]
$$

with $\partial_{\epsilon}(n+\epsilon m)=m$ for $n, m \in M_{i}$, and $R_{i}$ a $\mathbf{C}[\epsilon]$-linear map of order at most 2 on $M_{i}$. Thus $R_{i}$ is a second order differential operator involving only derivatives in the fibre directions (and not in the base direction $\epsilon$ ). The symbol of such a $D$ is the second order part of the $D_{i}$ (and thus of the $R_{i}$ ). The restriction of the symbol to $X\left(\subset X_{\epsilon}\right)$ is:

$$
\bar{\rho}(D):=\left\{\sigma\left(\bar{R}_{i}\right)\right\} \quad\left(\in H^{0}\left(X, S^{2} T_{X}\right)\right), \quad \text { where } \quad R_{i}=\bar{R}_{i}+\epsilon \bar{R}_{i}^{\prime}
$$

with $\bar{R}_{i}, \bar{R}_{i}^{\prime}: M_{i} \rightarrow M_{i}[\epsilon]$ (recall $R_{i}$ is $\epsilon$-linear). 
For any $s \in H^{0}(X, L)$ we then have the hypercohomology class $\delta_{s}(\bar{\rho}(D))=$ $\left(\left\{\bar{R}_{i}\left(s_{i}\right)\right\},\left\{\bar{R}_{i}-\bar{R}_{j}\right\}\right)\left(\in \mathbf{H}^{1}\left(d^{1} s\right)\right)$. Let us show that the deformation $\left(X_{\epsilon}, L_{\epsilon}\right)$ is the deformation determined by $-\beta\left(\delta_{s}(\bar{\rho}(D))\right)$ :

$$
\left[\left(X_{\epsilon}, L_{\epsilon}\right)\right]+\beta\left(\delta_{s}(\bar{\rho}(D))\right)=0 \quad\left(\in H^{1}\left(X, \operatorname{Diff}_{X}^{(1)}(L)\right)\right) .
$$

This can be verified as follows. Note that $\beta \delta_{s}(\bar{\rho}(D))=\left\{\bar{R}_{i}-\bar{R}_{j}\right\}$ and $\left[\left(X_{\epsilon}, L_{\epsilon}\right)\right]=$ $\left\{D_{i j}\right\}$. The fact that $D$ is an operator on $L_{\epsilon}$ implies that $D_{i} \circ\left(\mathrm{id}+\epsilon D_{i j}\right)=$ $\left(\right.$ id $\left.+\epsilon D_{i j}\right) \circ D_{j}$. Taking the "constant" term of this equation we obtain $\bar{R}_{i}+D_{i j}=\bar{R}_{j}$ as desired.

Next we consider any deformation $\left(X_{\epsilon}, L_{\epsilon}, s_{\epsilon}\right)$ of $(X, L, s)$. We say that a section $s_{\epsilon}=\left\{s_{i}+\epsilon t_{i}\right\} \in H^{0}\left(X_{\epsilon}, L_{\epsilon}\right)$ is flat for $D$ if

$$
D_{i}\left(s_{i}+\epsilon t_{i}\right) \equiv 0 \quad \bmod (\epsilon), \quad \text { equivalently } \quad t_{i}=-\bar{R}_{i}\left(s_{i}\right)
$$

in this case we write $\nabla(D)\left(s_{\epsilon}\right)=0$. Since $\left[\left(X_{\epsilon}, L_{\epsilon}, s_{\epsilon}\right)\right]=\left(\left\{t_{i}\right\},\left\{D_{i j}\right\}\right)\left(\in \mathbf{H}\left(d^{1} s\right)\right)$ we get:

$$
\delta_{s}(\bar{\rho}(D))=-\left[\left(X_{\epsilon}, L_{\epsilon}, s_{\epsilon}\right)\right] \quad \text { iff } \quad \nabla(D)\left(s_{\epsilon}\right)=0
$$

2.2.4. A remark on symbols. We use the conventions of [W] concerning differential operators and symbols. In particular when we write $S^{2} T_{X}$ we mean symmetric tensors in the sheaf of vector fields on $X$. Note that if $X=\mathbf{A}_{\mathbf{C}}^{1}$ with coordinate $t$, then the symbol of the operator $\partial^{2} / \partial t^{2}\left(\in \operatorname{Diff}_{\mathcal{O}}^{(1)}\right)$ is the symmetric section $2 \partial / \partial t \otimes \partial / \partial t\left(\in S^{2} T_{X}\right)$. By abuse of notation we will sometimes write $\sigma\left(\partial^{2} / \partial t^{2}\right)=$ $2 \partial^{2} / \partial t^{2}$.

\subsection{Heat operators.}

2.3.1. Notation. Let $p: \mathcal{X} \rightarrow S$ be a smooth surjective morphism of smooth varieties over $\mathbf{C}$. Let $\mathcal{L}$ be an invertible $\mathcal{O}_{\mathcal{X}}$-module over $\mathcal{X}$.

We write $\mathcal{T}_{\mathcal{X}}\left(\right.$ resp. $\mathcal{T}_{S}$, resp. $\left.\mathcal{T}_{\mathcal{X} / S}\right)$ for the sheaf of vector fields on $\mathcal{X}$ (resp. $S$, resp. $\mathcal{X}$ over $S)$. We denote by $\operatorname{Diff}_{\mathcal{X}}^{(k)}(\mathcal{L})$ the sheaf of differential operators of order at most $k$ on $\mathcal{L}$ over $\mathcal{X}$. Further, $\operatorname{Diff}_{\mathcal{X} / S}^{(k)}(\mathcal{L}) \subset \operatorname{Diff}_{\mathcal{X}}^{(k)}(\mathcal{L})$ denotes the subsheaf of $p^{-1}\left(\mathcal{O}_{S}\right)$-linear operators. We remark that $\operatorname{Diff}_{\mathcal{X}}^{(k)}(\mathcal{L})$ is a coherent (left) $\mathcal{O}_{\mathcal{X}}$ module and that $\operatorname{Diff}_{\mathcal{X} / S}^{(k)}(\mathcal{L})$ is a coherent submodule. For any $k$ there is a symbol map

$$
\sigma^{(k)}: \operatorname{Diff}_{\mathcal{X}}^{(k)}(\mathcal{L}) \longrightarrow S^{k} \mathcal{T}_{\mathcal{X}}=\operatorname{Sym}_{\mathcal{O}_{\mathcal{X}}}^{k}\left(\mathcal{T}_{\mathcal{X}}\right)
$$

It maps $\operatorname{Diff}_{\mathcal{X} / S}^{(k)}(\mathcal{L})$ into $S^{k} \mathcal{T}_{\mathcal{X} / S}$

Consider a point $x \in \mathcal{X}(\mathbf{C})$, an (étale or analytic) neighbourhood $U$ of $p(x)$ in $S$, and an (étale or analytic) neighbourhood $V$ of $x$ in $\mathcal{X}$ such that $p(V) \subset U$. Assume we have coordinate functions $t_{1}, \ldots, t_{r} \in \mathcal{O}_{S}(U)$ and functions $x_{1}, \ldots, x_{n} \in \mathcal{O}_{\mathcal{X}}(V)$ such that $t_{1}=t_{1} \circ p, \ldots, t_{r}=t_{r} \circ p, x_{1}, \ldots, x_{n}$ are coordinates on $V$. (In the étale case this means that $\Omega_{U}^{1}=\bigoplus \mathcal{O}_{U} \mathrm{~d} t_{i}$ and $\Omega_{V / U}^{1}=\bigoplus \mathcal{O}_{V} \mathrm{~d} x_{i}$.) Then the elements $\partial / \partial x_{i}$ form a basis for $\left.\mathcal{T}_{\mathcal{X} / S}\right|_{V}$ over $\mathcal{O}_{V}$. The system $\left(V, t_{1}, \ldots, t_{r}, x_{1}, \ldots, x_{n}\right)$ will be called a coordinate patch. We can find coordinate patches around any point $x \in \mathcal{X}(\mathbf{C})$. 
2.3.2. Definition. A heat operator $D$ on $\mathcal{L}$ over $S$ is a (left) $\mathcal{O}_{\mathcal{X}}$-module homomorphism

$$
D: p^{*}\left(\mathcal{T}_{S}\right) \longrightarrow \operatorname{Diff}_{\mathcal{X}}^{(2)}(\mathcal{L})
$$

which satisfies the following property: for any coordinate patch $\left(V, t_{1}, \ldots, t_{r}\right.$, $\left.x_{1}, \ldots, x_{n}\right)$ as above, and any trivialization $\left.\mathcal{L}\right|_{V} \cong \mathcal{O}_{V}$, the operator $D\left(\partial / \partial t_{1}\right)$ has the following shape:

$$
D\left(\frac{\partial}{\partial t_{1}}\right)=f+\frac{\partial}{\partial t_{1}}+\sum_{i=1}^{n} f_{i} \frac{\partial}{\partial x_{i}}+\sum_{1 \leq i \leq j \leq n} f_{i j} \frac{\partial^{2}}{\partial x_{i} \partial x_{j}}
$$

for certain $f, f_{i}, f_{i j} \in \mathcal{O}_{\mathcal{X}}(V)$. More precisely, let

$$
\mathcal{W}_{\mathcal{X} / S}(\mathcal{L})=\operatorname{Diff}_{\mathcal{X}}^{(1)}(\mathcal{L})+\operatorname{Diff}_{\mathcal{X} / S}^{(2)}(\mathcal{L}) \subset \operatorname{Diff}_{\mathcal{X}}^{(2)}(\mathcal{L})
$$

be the subsheaf of second order differential operators on $\mathcal{L}$ whose symbol lies in $S^{2}\left(\mathcal{T}_{\mathcal{X} / S}\right) \subset S^{2}\left(\mathcal{T}_{\mathcal{X}}\right)$. Note that $\operatorname{Difff}_{\mathcal{X} / S}^{(1)}(\mathcal{L}) \subset \mathcal{W}_{\mathcal{X} / S}(\mathcal{L})$ and that there is a canonical exact sequence

$$
0 \longrightarrow \operatorname{Diff}_{\mathcal{X} / S}^{(1)}(\mathcal{L}) \longrightarrow \mathcal{W}_{\mathcal{X} / S}(\mathcal{L}) \longrightarrow p^{*}\left(\mathcal{T}_{S}\right) \oplus S^{2} \mathcal{T}_{\mathcal{X} / S} \longrightarrow 0 .
$$

Our condition on $D$ above means that $D$ is a map $D: p^{*}\left(\mathcal{T}_{S}\right) \rightarrow \mathcal{W}_{\mathcal{X} / S}(\mathcal{L})$ whose composition with the map $\mathcal{W}_{\mathcal{X} / S}(\mathcal{L}) \rightarrow p^{*}\left(\mathcal{T}_{S}\right)$ is the identity. (So $D\left(\partial / \partial t_{1}\right)$ has no terms involving $\partial / \partial t_{i}$ except for the term $\partial / \partial t_{1}$ with coefficient 1.)

Such a map $D$ is determined by the $\mathcal{O}_{S}$-linear map $p_{*} D: \mathcal{T}_{S} \rightarrow p_{*}\left(\operatorname{Diff}_{\mathcal{X}}^{(2)}(\mathcal{L})\right)$. Note that $\mathcal{O}_{S}$ is a subsheaf of $p_{*}\left(\operatorname{Difff}_{\mathcal{X}}^{(2)}(\mathcal{L})\right)$. Any $\mathcal{O}_{S}$-linear map $\bar{D}: \mathcal{T}_{S} \rightarrow$ $p_{*}\left(\operatorname{Diff}_{\mathcal{X}}^{(2)}(\mathcal{L})\right) / \mathcal{O}_{S}$ can locally be lifted to a map into $p_{*}\left(\operatorname{Diff}_{\mathcal{X}}^{(2)}(\mathcal{L})\right)$. This means that any point $s \in S(\mathbf{C})$ has a neighbourhood $U$ such that there is an $\mathcal{O}_{U}$-linear map $D_{U}:\left.\left.\mathcal{T}_{S}\right|_{U} \rightarrow p_{*}\left(\operatorname{Diff}_{\mathcal{X}}^{(2)}(\mathcal{L})\right)\right|_{U}$ which reduces to $\left.\bar{D}\right|_{U}$.

A projective heat operator $\bar{D}$ on $\mathcal{L}$ over $S$ is an $\mathcal{O}_{S}$-linear map

$$
\bar{D}: \mathcal{T}_{S} \longrightarrow p_{*}\left(\operatorname{Diff}_{\mathcal{X}}^{(2)}(\mathcal{L})\right) / \mathcal{O}_{S}
$$

such that any local lifting $D_{U}$ as above gives rise to a heat operator on $\mathcal{L}$ over $U$.

The symbol of a heat operator $D$ is the $\mathcal{O}_{\mathcal{X}}$-linear map

$$
\rho_{D}: p^{*}\left(\mathcal{T}_{S}\right) \longrightarrow S^{2} \mathcal{T}_{\mathcal{X} / S}
$$

given by composing $D$ with the symbol $\sigma^{(2)}$. We note that the symbol of a projective heat operator is well-defined.

2.3.3. Heat operators and connections. We claim that a heat operator $D$ gives rise to a connection

$$
\nabla(D): p_{*}(\mathcal{L}) \longrightarrow p_{*}(\mathcal{L}) \otimes_{\mathcal{O}_{S}} \Omega_{S}^{1}
$$

Indeed, suppose that $s \in p_{*}(\mathcal{L})(U)=\mathcal{L}\left(p^{-1}(U)\right)$ and that $\theta \in \mathcal{T}_{S}(U)$. Then $D\left(p^{-1} \theta\right)$ is a second order differential operator on $\mathcal{L}$ over $p^{-1}(U)$. Hence

$$
D\left(p^{-1} \theta\right)(s) \in \mathcal{L}\left(p^{-1}(U)\right)=p_{*}(\mathcal{L})(U) .
$$

By our local description of $D$ above we see that $D\left(p^{-1} \theta\right)(f s)=f D\left(p^{-1} \theta\right)(s)+\theta(f) s$ for every $f \in \mathcal{O}_{S}(U)$. Hence the operation $(\theta, s) \mapsto D\left(p^{-1} \theta\right)(s)$ defines a connection $\nabla(D)$ on $p_{*} \mathcal{L}$ over $S$. In the sequel we will often write $D(\theta)$ instead of $D\left(p^{-1} \theta\right)$.

If we have a projective heat operator $\bar{D}$, the connection $\nabla(\bar{D})=\nabla\left(D_{U}\right)$ is only defined locally, by choosing lifts $D_{U}$. The difference of two local lifts is given by a local section $\eta \in \Omega_{S}^{1}(U)$, in which case the difference of the two connections $\nabla\left(D_{U}\right)$ 
is multiplication by $\eta$. Thus $\bar{D}$ defines unambiguously a projective connection $\bar{\nabla}(\bar{D})$.

2.3.4. Heat operators and deformations. Let $D$ be a heat operator on $\mathcal{L}$ over $S$. Let $0 \in S(\mathbf{C})$ be a point of the base. Put $X=\mathcal{X}_{0}$ and $L=\mathcal{L}_{0}$. An element $\theta \in T_{0} S$ will also be considered as a morphism $\theta: \operatorname{Spec}(\mathbf{C}[\epsilon]) \rightarrow S$. By base change we get a pair $\left(X_{\epsilon}, L_{\epsilon}\right)$ over $\operatorname{Spec}(\mathbf{C}[\epsilon])$. Clearly, $w_{\theta}:=\rho_{D}(\theta)$ is an element of $H^{0}\left(X, S^{2} T_{X}\right)$. It can be seen by working through the definitions that $\left[\left(X_{\epsilon}, L_{\epsilon}\right)\right]+\beta\left(\delta_{s}\left(w_{\theta}\right)\right)=0$ for any section $s \in H^{0}(X, L)$. (Note that in Subsection 2.2.2 we showed that $\beta\left(\delta_{s}\left(w_{\theta}\right)\right)$ was independent of $s$.) Let $s_{\epsilon} \in H^{0}\left(X_{\epsilon}, L_{\epsilon}\right)$ lift $s$. One can show that $\left[\left(X_{\epsilon}, L_{\epsilon}, s_{\epsilon}\right)\right]=-\delta_{s}\left(w_{\theta}\right)$ if and only if the section $s_{\epsilon}$ is horizontal for the connection $\nabla(D)$.

2.3.5. Projective heat operators and change of line bundle. Let $D$ be a heat operator on $\mathcal{L}$ over $S$. Let $g \in H^{0}\left(S, \mathcal{O}_{S}^{*}\right)$ be an invertible function on $S$. Multiplication by $p^{-1}(g)$ defines an invertible operator on $\mathcal{L}$, also denoted by $g$. For any local section $\theta$ of $\mathcal{T}_{S}$, we have the obvious relation

$$
g^{-1} \circ D(\theta) \circ g=g^{-1} \theta(g)+D(\theta) .
$$

We conclude that $g^{-1} \circ D \circ g$ is a heat operator and that the projective heat operators $\bar{D}$ and $\overline{g^{-1} \circ D \circ g}$ are equal. (The difference is given by the section $g^{-1} \mathrm{~d} g=\mathrm{d} \log g$ of $\Omega_{S}^{1}$.)

Suppose that $\mathcal{M}$ is an invertible $\mathcal{O}_{S}$-module on $S$. The above implies that the set of projective heat operators on $\mathcal{L}$ over $S$ can be identified canonically with the set of projective heat operators on $\mathcal{L}^{\prime}=\mathcal{L} \otimes_{\mathcal{O}_{\mathcal{X}}} p^{*}(\mathcal{M})$. Indeed, choose a covering of $S$ on whose members $U_{i}$ the line bundle $\mathcal{M}$ becomes trivial. This identifies the heat operators on $\mathcal{L}$ and $\mathcal{L}^{\prime}$ over $U$. The difference in the local identifications is given by the 1 -forms $\mathrm{d} \log g_{i j} \in \Omega_{U_{i j}}^{1}$.

2.3.6. Heat operators and flatness. We say that a heat operator $D$ on $\mathcal{L}$ over $S$ is flat if $D\left(\left[\theta, \theta^{\prime}\right]\right)=\left[D(\theta), D\left(\theta^{\prime}\right)\right]$ for any two local sections $\theta, \theta^{\prime}$ of $\mathcal{T}_{S}$ on $U \subset S$. It suffices to consider local vector fields $\theta, \theta^{\prime}$ on $S$ with $\left[\theta, \theta^{\prime}\right]=0$ and to check that $\left[D(\theta), D\left(\theta^{\prime}\right)\right]=0$. We remark that in this case the operator $\left[D(\theta), D\left(\theta^{\prime}\right)\right]$ is a section of $\operatorname{Diff}_{\mathcal{X} / S}^{(3)}(\mathcal{L})$ over $p^{-1}(U)$. We say that $D$ is projectively flat if we have $D\left(\left[\theta, \theta^{\prime}\right]\right)=h+\left[D(\theta), D\left(\theta^{\prime}\right)\right]$ for some function $h=h_{\theta, \theta^{\prime}} \in \mathcal{O}_{S}(U)$ for any $\theta, \theta^{\prime} \in \mathcal{T}_{S}(U)$. A projective heat operator $\bar{D}$ is called projectively flat if any of the local lifts $D_{U}$ are projectively flat.

We remark that if the heat operator $D$ is flat, then the associated connection $\nabla(D)$ on $p_{*} \mathcal{L}$ is flat as well. In particular, if $p_{*} \mathcal{L}$ is a coherent $\mathcal{O}_{S}$-module, then this implies that $p_{*} \mathcal{L}$ is locally free. In the same vein we have that a projectively flat projective heat operator $\bar{D}$ defines a projectively flat projective connection $\bar{\nabla}(\bar{D})$ on $p_{*} \mathcal{L}$.

2.3.7. Heat operators with given symbol. Let $\mathcal{X} \rightarrow S$ and $\mathcal{L}$ be as above, and assume that a $\mathcal{O}_{\mathcal{X}}$-linear map $\rho: p^{*} \mathcal{T}_{S} \rightarrow S^{2} \mathcal{T}_{\mathcal{X} / S}$ is given. The question we are going to study is the following:

When can we find a (projective) heat operator $D$ on $\mathcal{L}$ over $S, D: p^{*} \mathcal{T}_{S} \rightarrow$ $\mathcal{W}_{\mathcal{X} / S}\left(\subset \operatorname{Diff}_{\mathcal{X}}^{(2)}(\mathcal{L})\right)$ with $\rho_{D}=\rho ?$

So the symbol $\rho_{D}$ (which is the composition of $D$ with the map $\sigma^{(2)}: \operatorname{Diff}_{\mathcal{X}}^{(2)}(\mathcal{L}) \rightarrow$ $\left.S^{2} \mathcal{T}_{\mathcal{X} / S}\right)$ should be equal to the given $\rho$. 
Let us define a number of canonical maps associated to the situation. First we have the standard exact sequence

$$
0 \longrightarrow \mathcal{T}_{\mathcal{X} / S} \longrightarrow \mathcal{T}_{\mathcal{X}} \longrightarrow p^{*} \mathcal{T}_{S} \longrightarrow 0
$$

This gives us an $\mathcal{O}_{S}$-linear map (the Kodaira-Spencer map)

$$
\kappa_{\mathcal{X} / S}: \mathcal{T}_{S} \longrightarrow R^{1} p_{*} \mathcal{T}_{\mathcal{X} / S}
$$

Next we have the exact sequence

$$
0 \longrightarrow \mathcal{T}_{\mathcal{X} / S} \longrightarrow \operatorname{Diff}_{\mathcal{X} / S}^{(2)}(\mathcal{L}) / \mathcal{O}_{\mathcal{X}} \longrightarrow S^{2} \mathcal{T}_{\mathcal{X} / S} \longrightarrow 0
$$

This gives an $\mathcal{O}_{S}$-linear map

$$
\mu_{\mathcal{L}} \quad: \quad p_{*} S^{2} \mathcal{T}_{\mathcal{X} / S} \longrightarrow R^{1} p_{*} \mathcal{T}_{\mathcal{X} / S}
$$

The invertible $\mathcal{O}_{\mathcal{X}}$-module $\mathcal{L}$ is given by an element of $H^{1}\left(\mathcal{X}, \mathcal{O}_{\mathcal{X}}^{*}\right)$. Using the map $\mathrm{d} \log : \mathcal{O}_{\mathcal{X}}^{*} \rightarrow \Omega_{\mathcal{X} / S}^{1}$ we get an element of $H^{1}\left(\mathcal{X}, \Omega_{\mathcal{X} / S}^{1}\right)$, and hence a section $[\mathcal{L}]$ in $H^{0}\left(S, R^{1} p_{*}\left(\Omega_{\mathcal{X} / S}^{1}\right)\right)$. This will be called the cohomology class of $\mathcal{L}$. By cup-product with $[\mathcal{L}]$ we get another map $p_{*} S^{2} \mathcal{T}_{\mathcal{X} / S} \rightarrow R^{1} p_{*} \mathcal{T}_{\mathcal{X} / S}, w \mapsto w \cup[\mathcal{L}]$. (There is a natural pairing $S^{2} \mathcal{T}_{\mathcal{X} / S} \otimes \Omega_{\mathcal{X} / S}^{1} \rightarrow \mathcal{T}_{\mathcal{X} / S}$.) It is shown in [W], 1.16, that we have $\mu_{\mathcal{L}}(w)=-w \cup[\mathcal{L}]+\mu_{\mathcal{O}}(w)$.

Now let $\theta$ be a local vector field on $S$, i.e., $\theta \in \mathcal{T}_{S}(U)$, with $U$ affine. We want to find a $D(\theta) \in \mathcal{W}_{\mathcal{X} / S}(\mathcal{L})$ with symbol $\rho(\theta)$. Recall that $\mathcal{W}_{\mathcal{X} / S}(\mathcal{L})$ is an extension:

$$
0 \longrightarrow \operatorname{Diff}_{\mathcal{X} / S}^{(1)}(\mathcal{L}) \longrightarrow \mathcal{W}_{\mathcal{X} / S}(\mathcal{L}) \longrightarrow p^{*}\left(\mathcal{T}_{S}\right) \oplus S^{2} \mathcal{T}_{\mathcal{X} / S} \longrightarrow 0 .
$$

The obstruction against finding a lift $D(\theta)\left(\in \mathcal{W}_{\mathcal{X} / S}(\mathcal{L})\right)$ of the section $p^{-1}(\theta) \oplus$ $\rho\left(p^{-1} \theta\right)$ of $p^{*} \mathcal{T}_{S} \oplus S^{2} \mathcal{T}_{\mathcal{X} / S}$ over $p^{-1}(U)$ is an element $o(\theta, \mathcal{L})$ in

$$
H^{1}\left(p^{-1}(U), \operatorname{Difff}_{\mathcal{X} / S}^{(1)}(\mathcal{L})\right)=H^{0}\left(U, R^{1} p_{*}\left(\operatorname{Difff}_{\mathcal{X} / S}^{(1)}(\mathcal{L})\right)\right)
$$

(recall that $U$ was assumed affine). There is an exact sequence

$$
0 \longrightarrow \mathcal{O}_{\mathcal{X}} \longrightarrow \operatorname{Diff}_{\mathcal{X} / S}^{(1)}(\mathcal{L}) \longrightarrow \mathcal{T}_{\mathcal{X} / S} \longrightarrow 0 \text {. }
$$

We remark that the image of the class $o(\theta, \mathcal{L})$ in $R^{1} p_{*} \mathcal{T}_{\mathcal{X} / S}(U)$ is the section $\kappa_{\mathcal{X} / S}(\theta)+\mu_{\mathcal{L}}(\rho(\theta))$. The first condition that has to be satisfied for $\bar{D}$ to exist is therefore:

$$
\kappa_{\mathcal{X} / S}+\mu_{\mathcal{L}} \circ \rho=0 .
$$

If this condition is satisfied, then we can find a lift of $p^{-1}(\theta) \oplus \rho\left(p^{-1} \theta\right)$ to an element $\bar{D}_{\theta}$ in the sheaf $\mathcal{W}_{\mathcal{X} / S}(\mathcal{L}) / \mathcal{O}_{\mathcal{X}}$. This element is well defined up to addition of a section of $\mathcal{T}_{\mathcal{X} / S}$. Hence we get a second obstruction in

$$
\operatorname{Coker}\left(p_{*} \mathcal{T}_{\mathcal{X} / S} \stackrel{[\mathcal{L}] \cup}{\longrightarrow} R^{1} p_{*} \mathcal{O}_{X}\right) .
$$

Let us assume for the moment that both obstructions vanish for any $\theta$ as above. Then we can locally find a $D(\theta)$ lifting the element $p^{-1}(\theta) \oplus \rho\left(p^{-1} \theta\right)$. Thus, if we have a basis $\theta_{1}, \ldots, \theta_{r}$ of $\mathcal{T}_{S}$ over $U$, then we can define $D_{U}$ by the formula $D_{U}\left(\sum a_{i} \theta_{i}\right)=\sum a_{i} D\left(\theta_{i}\right)$ for certain choices of the elements $D\left(\theta_{i}\right)$. If there is some way of choosing the elements $D(\theta)$ uniquely up to an element of $p^{-1} \mathcal{O}_{S}(U)$, e.g. if the map in $(* *)$ is injective and $p_{*}\left(\mathcal{O}_{\mathcal{X}}\right)=\mathcal{O}_{S}$, then $D_{U}$ determines a unique projective heat operator over $U$. In this case the map $\rho$ determines a unique projective heat operator on $\mathcal{L}$ over $S$. 
Here is another set of hypotheses which imply the existence of heat operators with given symbol. Let $0 \in S(\mathbf{C})$ be a point. Put $X=\mathcal{X}_{0}$ and $L=\mathcal{L}_{0}$ as in 2.3.4. Let $\theta \in T_{0} S$ and consider $\left(X_{\epsilon}, L_{\epsilon}\right)$ over $\operatorname{Spec}(\mathbf{C}[\epsilon])$ as in 2.3.4. We know from 2.3.4 that $\left[\left(X_{\epsilon}, L_{\epsilon}\right)\right]+\beta\left(\delta_{s}(\rho(\theta))\right)=0$ if a heat operator exists. Suppose that

1. $R^{1} p_{*} \mathcal{O}_{\mathcal{X}}$ and $R^{1} p_{*} \mathcal{T}_{\mathcal{X} / S}$ are locally free on $S$, and have fibres $H^{1}\left(X, \mathcal{O}_{X}\right)$ and $H^{1}\left(X, T_{X}\right)$ at any point 0 in $S(\mathbf{C})$.

2. for any 0 and $\theta$ as above, we have $\left[\left(X_{\epsilon}, L_{\epsilon}\right)\right]+\beta\left(\delta_{s}(\rho(\theta))\right)=0$.

If these conditions are satisfied, then we can at least find local liftings $D_{U}$ of $\rho$ to a heat operator. Again, we will get a "canonical" projective heat operator if there is a "preferred" way of choosing the local lifts $D\left(\theta_{i}\right)$.

2.3.8. Heat operators on abelian schemes. In this subsection, we let $\mathcal{X} \rightarrow S$ be an abelian scheme with zero section $o: S \rightarrow \mathcal{X}$. We assume the line bundle $\mathcal{L}$ is relatively ample on $\mathcal{X}$ over $S$, i.e., $\mathcal{L}$ defines a polarization of $\mathcal{X}$ over $S$. We will show that there exists a unique projective heat operator on $\mathcal{L}$ over $S$; see [W].

We first remark that in this case the map $(* *)$ of 2.3.7 is an isomorphism. This implies that $\Gamma\left(p^{-1}(U), \operatorname{Diff}_{\mathcal{X} / S}^{(1)}(\mathcal{L})\right)=\mathcal{O}_{S}(U)$ for any $U \subset S$. Hence, by the discussions in 2.3.7 we get a unique heat operator as soon as we have a (symbol) map $\rho$ satisfying $(* *)$.

We have $\mu_{\mathcal{O}}=0$ in this situation, as we can let elements of $S^{2} \mathcal{T}_{\mathcal{X} / S}$ act by translation invariant operators (see $[\mathrm{W}], 1.20)$. Hence, $\mu_{\mathcal{L}}(-)=-[\mathcal{L}] \cup(-)$. Now we use that

$$
p_{*}\left(\otimes^{2} \mathcal{T}_{\mathcal{X} / S}\right) \stackrel{[\mathcal{L}] \cup}{\longrightarrow} R^{1} p_{*} \mathcal{T}_{\mathcal{X} / S} \cong o^{*} \mathcal{T}_{\mathcal{X} / S} \otimes R^{1} p_{*} \mathcal{O}_{\mathcal{X}}
$$

is an isomorphism. Consider $\rho:=([\mathcal{L}] \cup)^{-1} \circ \kappa_{\mathcal{X} / S}$. It is well known that this is a map into $p_{*} S^{2} \mathcal{T}_{\mathcal{X} / S}$ and it solves (*) by definition; it is of course the unique solution to $(*)$. The unique projective heat operator $\bar{D}=\bar{D}_{\mathcal{L}}$ with $\rho=\rho_{\bar{D}}$ is called the projective heat operator associated to $(\mathcal{X} / S, \mathcal{L})$.

We make the remark that if $\mathcal{L}^{\prime}=\mathcal{L}^{\otimes n}$, then we have $\rho_{\bar{D}^{\prime}}=(1 / n) \rho_{\bar{D}}$. It is known that these projective heat operators are projectively flat. This can be seen in a number of ways; one way is to show that $p_{*}\left(\operatorname{Diff}_{\mathcal{X} / S}^{(3)}(\mathcal{L})\right)=\mathcal{O}_{S}$ in this case; compare 2.3.6.

Uniqueness of the construction of $\bar{D}$ implies that $\bar{D}$ commutes (projectively) with the action of the theta-group $\mathcal{G}(\mathcal{L})$. In particular the projective monodromy group of the (projectively flat) connection $\bar{\nabla}(\bar{D})$ on $\left(p_{*} \mathcal{L}\right)_{0}$ normalizes the action of $\mathcal{G}(L)$.

Recall that the classical theta functions satisfy a heat equation. This is exactly the heat equation above where the base is the Siegel upper half space.

2.3.9. Heat operators and functoriality. Let $\mathcal{X} \rightarrow S$ and $\mathcal{L}$ be as above, and let $D$ be a heat operator on $\mathcal{L}$ over $S$. Note that for any morphism of smooth schemes $\varphi: S^{\prime} \rightarrow S$ there is a pullback heat operator $D^{\prime}=\varphi^{*}(D)$ on the pullback $\mathcal{L}^{\prime}$ on $\mathcal{X}^{\prime}=S^{\prime} \times{ }_{S} \mathcal{X}$. We leave it to the reader to give the precise definition. This pullback preserves flatness. If $\varphi^{*}\left(p_{*} \mathcal{L}\right) \cong p_{*}^{\prime} \mathcal{L}^{\prime}$, then the connection $\nabla\left(D^{\prime}\right)$ is the pullback of the connection $\nabla(D)$. In addition pullback is well defined for projective heat operators and preserves projective flatness.

If $\psi: \mathcal{U} \rightarrow \mathcal{X}$ is an étale morphism of schemes over $S$, then the heat operator $D$ induces a heat operator $\psi^{*}(D)$ on $\psi^{*}(\mathcal{L})$ over $S$. 
2.3.10. Heat operators and compatibility. Suppose we have a second smooth surjective morphism $q: \mathcal{Y} \rightarrow S$ and a morphism $f: \mathcal{Y} \rightarrow \mathcal{X}$ over $S$. We will assume that $f$ is a submersion. This means that for any point $y \in \mathcal{Y}(\mathbf{C})$ there is a coordinate patch $\left(V, t_{1}, \ldots, t_{r}, x_{1}, \ldots, x_{c}, y_{1}, \ldots, y_{m}\right)$ of $f(y)$ in $\mathcal{X}$ such that $\left(f^{-1}(V), t_{1}, \ldots, t_{r}, y_{1}=y_{1} \circ f, \ldots, y_{m}=y_{m} \circ f\right)$ is a coordinate patch for $y$ on $\mathcal{Y}$ and $x_{i} \circ f=0$.

Let $\mathcal{L}$ be a line bundle on $\mathcal{X}$ as before. Let us say that a (projective) heat operator $D$ on $\mathcal{L}$ over $S$ is (weakly) compatible with $f: \mathcal{Y} \rightarrow \mathcal{X}$ if whenever we have a coordinate patch $\left(V, t_{1}, \ldots, t_{r}, x_{1}, \ldots, x_{c}, y_{1}, \ldots, y_{m}\right)$ as above, and a trivialization $\left.\mathcal{L}\right|_{V} \cong \mathcal{O}_{V}$, then the operator $D\left(\partial / \partial t_{1}\right)$ has the following symbol:

$$
\rho_{D}\left(\frac{\partial}{\partial t_{1}}\right)=\sum_{i \leq j=1}^{m} f_{i j} \frac{\partial^{2}}{\partial y_{i} \partial y_{j}}+\sum_{i=1}^{c} x_{i} \Xi_{i}
$$

for certain $f_{i j} \in \mathcal{O}_{\mathcal{X}}(V)$ and $\Xi_{i} \in S^{2} \mathcal{T}_{\mathcal{X} / S}(V)$. More precisely, this means there exists a $\rho=\rho_{f, D}: q^{*} \mathcal{T}_{S} \rightarrow S^{2} \mathcal{T}_{\mathcal{Y} / S}$ such that the following diagram commutes:

$$
\begin{array}{rcccc}
f^{*}\left(\rho_{D}\right) & : & f^{*} p^{*} \mathcal{T}_{S} & \longrightarrow & f^{*} S^{2} \mathcal{T}_{\mathcal{X} / S} \\
& & \| & & \uparrow \\
\rho_{f, D} & : & q^{*} \mathcal{T}_{S} & \longrightarrow & S^{2} \mathcal{T}_{\mathcal{Y} / S}
\end{array}
$$

Suppose that $D^{\prime}$ is a heat operator on $f^{*} \mathcal{L}$ on $\mathcal{Y}$ over $S$. We say that $D^{\prime}$ is (weakly) compatible with $D$ if $D$ is (weakly) compatible with $f$ and we have $\rho_{D^{\prime}}=\rho_{f, D}$. In this case it is not true in general that $\left.D(\theta)(s)\right|_{\mathcal{Y}}=D^{\prime}(\theta)\left(\left.s\right|_{\mathcal{Y}}\right)$.

Assume that $D$ is compatible with $f$. Let us write $N_{\mathcal{Y}} \mathcal{X}$ for the generalized normal bundle of $\mathcal{Y}$ in $\mathcal{X}$, i.e., $N_{\mathcal{Y}} \mathcal{X}=\operatorname{Coker}\left(\mathcal{T}_{\mathcal{Y} / S} \rightarrow f^{*} \mathcal{T}_{\mathcal{X} / S}\right)$. Choose a local coordinate patch $\left(f^{-1}(V), t_{1}, \ldots, t_{r}, y_{1}=y_{1} \circ f, \ldots, y_{m}=y_{m} \circ f\right)$ as above. Write the operator $D\left(\partial / \partial t_{1}\right)$ in the form

$$
D\left(\frac{\partial}{\partial t_{1}}\right)=f+\frac{\partial}{\partial t_{1}}+\sum_{i=1}^{c} f_{i} \frac{\partial}{\partial x_{i}}+\sum_{i=1}^{m} g_{i} \frac{\partial}{\partial y_{i}}+\sum_{i \leq j=1}^{m} f_{i j} \frac{\partial^{2}}{\partial y_{i} \partial y_{j}}+\sum_{i=1}^{c} x_{i} \Xi_{i}
$$

for certain local functions $f, f_{i}, g_{i}, f_{i j}$ and second order operators $\Xi_{i}$. It can be seen (and we leave this to the reader) that the class of $\sum f_{i} \frac{\partial}{\partial x_{i}}$ in $N_{\mathcal{Y}} \mathcal{X}\left(f^{-1}(V)\right)$ is independent of the choice of the local trivialization and coordinates. The upshot of this is that if $D$ is compatible with $\mathcal{Y} \rightarrow \mathcal{X}$, then there is a first order symbol

$$
\sigma_{f, D}: q^{*} \mathcal{T}_{S} \longrightarrow N_{\mathcal{Y}} \mathcal{X}
$$

We say that $D$ is strictly compatible with $f: \mathcal{Y} \rightarrow \mathcal{X}$ if $D$ is compatible with $f$ and the symbol $\sigma_{f, D}$ is zero. We remark that this notion is well defined for a projective heat operator as well.

Assume that $D$ is strictly compatible with $\mathcal{Y} \rightarrow \mathcal{X}$. It follows from the local description of our compatibility of restrictions that $D$ induces a heat operator $D_{\mathcal{Y}}$ on the invertible $\mathcal{O}_{\mathcal{Y}}$-module $f^{*} \mathcal{L}$ over $S$. It is characterized by the property $\left.D(\theta)(s)\right|_{\mathcal{Y}}=D_{\mathcal{Y}}(\theta)\left(\left.s\right|_{\mathcal{Y}}\right)$ for any local section $\theta$ of $\mathcal{T}_{S}$ and any local section $s$ of $\mathcal{L}$. It is clear that the symbol of $D_{\mathcal{Y}}$ is equal to $\rho_{f, D}$, hence that $D_{\mathcal{Y}}$ is compatible with $D$. We say that a heat operator $D^{\prime}$ on $f^{*} \mathcal{L}$ is strictly compatible with $D$ if $D$ is strictly compatible with $f: \mathcal{Y} \rightarrow \mathcal{X}$ and $D^{\prime}=D \mathcal{Y}$. In this case the restriction map

$$
p_{*} \mathcal{L} \longrightarrow q_{*} f^{*} \mathcal{L}
$$


is horizontal for the connections induced by $D$ and $D^{\prime}=D_{\mathcal{Y}}$. Note that $D_{\mathcal{Y}}$ is flat if $D$ is flat. Similar remarks hold for projective heat operators and projective flatness.

\subsection{Moduli of bundles.}

2.4.1. The symbol $\rho_{\text {Hitchin }}$ in terms of moduli of bundles. In this subsection we explain how to get a symbol map $\rho$ as in 2.3.7 in the case of the relative moduli space of rank 2 bundles of a family of curves.

Let $C$ be a smooth projective curve over $\operatorname{Spec}(\mathbf{C})$, and let $E$ be a stable $\mathcal{O}_{C^{-}}$ module of $\operatorname{rank} 2$ with $\operatorname{det}(E) \cong \mathcal{O}_{C}$. This gives a point $[E] \in \mathcal{M}_{C}(\mathbf{C})$. There are canonical identifications

$$
T_{[E]} \mathcal{M}_{C}=H^{1}\left(C, \mathcal{E} n d_{0}(E)\right) \quad \text { and } \quad T_{[E]}^{*} \mathcal{M}_{C}=H^{0}\left(C, \mathcal{E} n d_{0}(E) \otimes \Omega_{C}^{1}\right)
$$

where $\mathcal{E} n d_{0}(E)$ denotes the sheaf of endomorphisms of $E$ with trace zero. A cotangent vector $\phi \in T_{[E]}^{*} \mathcal{M}_{C}$ thus corresponds to a homomorphism of $\mathcal{O}_{C}$-modules $\phi: E \rightarrow E \otimes \Omega_{C}^{1}$. (Note that this is a Higgs field on the bundle $E$; see [Si].) Composing and taking the trace gives a symmetric bilinear pairing

$$
\begin{aligned}
& T_{[E]}^{*} \mathcal{M}_{C} \times T_{[E]}^{*} \mathcal{M}_{C} \longrightarrow H^{0}\left(C,\left(\Omega_{C}^{1}\right)^{\otimes 2}\right) \\
&(\phi, \psi) \longmapsto \\
& \operatorname{Trace}(\phi \circ \psi) .
\end{aligned}
$$

We dualize this and use Serre duality to obtain a map

$$
\rho_{C, E}: H^{1}\left(C, T_{C}\right) \longrightarrow S^{2} T_{[E]} \mathcal{M}_{C} .
$$

Let us make the observation that the construction above can be performed in families. Let $\pi: \mathcal{C} \rightarrow S$ be a smooth projective family of curves over the scheme $S$ smooth over $\operatorname{Spec}(\mathbf{C})$. Let $p: \mathcal{M} \rightarrow S$ be the associated family of moduli spaces of rank 2 semi-stable bundles with trivial determinant up to $S$-equivalence. We remark that $p$ is a flat projective morphism, not smooth in general. However, the open part of stable bundles $\mathcal{M}^{s} \subset \mathcal{M}$ is smooth over $S$. We denote this smooth morphism by $p^{s}: \mathcal{M}^{s} \rightarrow S$.

Now let $T \rightarrow S$ be a morphism of finite type. We denote by an index ${ }_{T}$ base change to $T$. Let $\mathcal{E}$ be a locally free sheaf of rank 2 on $\mathcal{C}_{T}$. For a point $0 \in T(\mathbf{C})$ we put $C=\mathcal{C}_{0}$ and $E=\mathcal{E}_{0}=\left.\mathcal{E}\right|_{C}$. Suppose that for any 0 we have (a) $\operatorname{det}(E) \cong \mathcal{O}_{C}$, and (b) the bundle $E$ is stable. In this case we get an $S$-morphism $t: T \rightarrow \mathcal{M}^{s}$. Analogously to the above we have a canonical isomorphism

$$
t^{*}\left(\Omega_{\mathcal{M}^{s} / S}^{1}\right) \cong\left(\pi_{T}\right)_{*}\left(\mathcal{E} n d_{0}(\mathcal{E}) \otimes_{\mathcal{O}_{\mathcal{C}_{T}}} \Omega_{\mathcal{C}_{T} / T}^{1}\right) .
$$

Using the same pairing and dualities as above we get an $\mathcal{O}_{T}$-linear map

$$
R^{1} \pi_{*} \mathcal{T}_{\mathcal{C}_{T} / T} \longrightarrow S^{2}\left(t^{*}\left(\mathcal{T}_{\mathcal{M}^{s} / S}^{1}\right)\right)
$$

If we compose this with the pullback to $T$ of the Kodaira-Spencer map $\mathcal{T}_{S} \rightarrow$ $R^{1} \pi_{*} \mathcal{I}_{\mathcal{C} / S}$ of the family $\mathcal{C} \rightarrow S$, then we get a map

$$
\rho_{\mathcal{E} / \mathcal{C}_{T}}: \mathcal{T}_{S} \otimes \mathcal{O}_{T} \longrightarrow S^{2}\left(t^{*}\left(\mathcal{T}_{\mathcal{M}^{s} / S}^{1}\right)\right) .
$$

If there existed a universal bundle $\mathcal{E}^{\text {univ }}$ over $\mathcal{M}^{s}$, then we would get a "symbol"

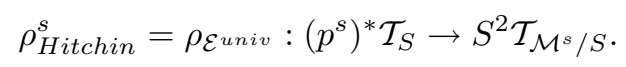


Although the universal bundle does not exist, the symbol $\rho_{\text {Hitchin }}^{s}$ does: it is the unique $\mathcal{O}_{\mathcal{M}^{s}}$-linear map such that, whenever $\mathcal{E} / \mathcal{C}_{T}$ is given, the pullback of $\rho_{\text {Hitchin }}$ to $T$ agrees with $\rho_{\mathcal{E} / \mathcal{C}_{T}}^{s}$. Uniqueness and existence follows from the existence of "universal" bundles étale locally over $\mathcal{M}^{s}$ and the invariance of $\rho_{\mathcal{E} / \mathcal{C}_{T}}$ under automorphisms of $\mathcal{E}$ over $\mathcal{C}_{T}$.

2.4.2. Hitchin's results in genus at least 3. Let us get back to our curve $C$ over $\operatorname{Spec}(\mathbf{C})$. We see from the above that any deformation $C_{\epsilon}$ of the curve will give a tensor in $\Gamma\left(\mathcal{M}_{C}^{s}, S^{2} \mathcal{T}_{\mathcal{M}_{C}}\right)$. However, since $\mathcal{M}_{C}$ has points which do not correspond to stable bundles (and these points are singular points of $\mathcal{M}_{C}$ for $g>2$ ) such a deformation does not give a global tensor in $H^{0}\left(\mathcal{M}_{C}, S^{2} T_{\mathcal{M}_{C}}\right)$. In [Hi], Section 5, one can find a sketch of a proof that the tensor extends if $g>2$ (or rank $>2$, a case we do not even consider, although our present considerations work in that case as well).

In the situation $\mathcal{C} \rightarrow S$ there is a canonical line bundle $\mathcal{L}$ on $\mathcal{M}$; it can be defined by the formula

$$
\mathcal{L}=\operatorname{det} R\left(\mathrm{pr}_{2}\right)_{*} \mathcal{E}^{\text {univ }}, \quad \operatorname{pr}_{2}: \mathcal{C} \times_{S} \mathcal{M} \rightarrow \mathcal{M}
$$

It is shown in [Hi], Theorem 3.6, Section 5 , that if $g>2$ the tensor $2 /(2 k+\lambda) \rho_{H i t c h i n}$ is the symbol of a unique projective flat heat operator $\bar{D}_{\text {Hitchin }}$ on $\mathcal{L}^{\otimes k}$ over $S$. (We remark that the extra factor 2 comes from our way of normalizing symbols; see Subsection 2.2.4.) In fact the arguments of [Hi] prove the existence of this heat operator over the moduli space of curves of genus $g>2$.

We will not use these results.

\subsection{The case of genus two curves.}

2.5.1. Notation in genus 2 case. We fix a scheme $S$ smooth over $\operatorname{Spec}(\mathbf{C})$ and a smooth projective morphism $\pi: \mathcal{C} \rightarrow S$, whose fibres $C$ are curves of genus 2 . We write $0 \in S(\mathbf{C})$ for a typical point and $C=\mathcal{C}_{0}$.

We introduce the following objects associated to the situation. $\mathrm{Pic}^{1}=\mathrm{Pic}_{\mathcal{C} / S}^{1} \rightarrow$ $S$ denotes the Picard scheme of invertible $\mathcal{O}_{\mathcal{C}}$ bundles of relative degree 1 on $\mathcal{C}$ over $S$. There is a natural morphism $\mathcal{C} \rightarrow \mathrm{Pic}^{1}$ over $S$, given by $P \in C(\mathbf{C})$ maps to $\left[\mathcal{O}_{C}(P)\right]$ in $\left(\mathrm{Pic}^{1}\right)_{0}(\mathbf{C})=\mathrm{Pic}^{1}(C)$. The image of this morphism is a relative divisor $\Theta^{1}=\Theta_{\mathcal{C} / S}^{1}$ on $\operatorname{Pic}^{1}$ over $S$; of course $\Theta^{1} \cong \mathcal{C}$ as $S$-schemes. Let us denote by $\alpha: \operatorname{Pic}^{1} \rightarrow S$ the structural morphism. Note that $\alpha_{*} \mathcal{O}_{\mathrm{Pic}^{1}}\left(2 \Theta^{1}\right)$ is a locally free $\mathcal{O}_{S}$-module of rank 4 on $S$. We put $\mathbf{P}=\left|2 \Theta^{1}\right|$ equal to the projective space of lines in this locally free sheaf. More precisely, we define

$$
p: \mathbf{P}=\mathbf{P}\left(\mathcal{H}_{\text {om }_{\mathcal{O}_{S}}}\left(\alpha_{*} \mathcal{O}_{\mathrm{Pic}^{1}}\left(2 \Theta^{1}\right), \mathcal{O}_{S}\right)\right) \longrightarrow S
$$

see [Ha], page 162 , for notation used. For a point $0 \in S(\mathbf{C})$ we put $P=\mathbf{P}_{0}$.

Let $T \rightarrow S$ be a morphism of finite type, and let $\mathcal{E}$ be a locally free sheaf of $\mathcal{O}_{\mathcal{C}_{T}}$-modules of rank 2 . For a point $0 \in T(\mathbf{C})$ we put $C=\mathcal{C}_{0}$ and $E=\mathcal{E}_{0}=\left.\mathcal{E}\right|_{C}$. Suppose that for any 0 we have

(a) $\operatorname{det}(E) \cong \mathcal{O}_{C}$,

(b) the bundle $E$ is semi-stable.

(Compare with Subsection 2.4.1.) We recall the relative divisor $\mathcal{D}_{\mathcal{E}} \subset \mathrm{Pic}_{T}^{1}$ associated to $\mathcal{E}$. Set-theoretically it has the following description:

$$
D_{E}:=\mathcal{D}_{\mathcal{E}} \cap \operatorname{Pic}^{1}(C)=\left\{[L] \in \operatorname{Pic}^{1}(C): \operatorname{dim} H^{1}(C, E \otimes L) \geq 1\right\} .
$$


To construct $\mathcal{D}_{\mathcal{E}}$ as a closed subscheme we may work étale locally on $T$. Hence we may assume that a Poincaré line bundle $\mathcal{L}$ on $\mathcal{C}_{T} \times_{T} \mathrm{Pic}_{T}^{1}$ exists, i.e., which has relative degree 1 for $p_{2}: \mathcal{C}_{T} \times_{T} \operatorname{Pic}_{T}^{1} \rightarrow \operatorname{Pic}_{T}^{1}$ and induces id $: \operatorname{Pic}_{T}^{1} \rightarrow \operatorname{Pic}_{T}^{1}$. We consider the line bundle

$$
\mathcal{N}=\left(\operatorname{det} R\left(p_{2}\right)_{*}\left(p_{1}^{*}(\mathcal{E}) \otimes \mathcal{L}\right)\right)^{\otimes-1}
$$

on $\operatorname{Pic}_{T}^{1}$. This line bundle has a natural section (the theta function) $\theta(\mathcal{E})$, which extends the section 1 on the open schematically dense subscheme $U \subset \operatorname{Pic}_{T}^{1}$ over which the complex $R\left(p_{2}\right)_{*}\left(p_{1}^{*}(\mathcal{E}) \otimes \mathcal{L}\right)$ is trivial. We define $\mathcal{D}_{\mathcal{E}}$ to be the zero set of the section $\theta(\mathcal{E})$; it is also the largest closed subscheme of $\mathrm{Pic}_{T}^{1}$ over which the coherent $\mathcal{O}_{\mathrm{Pic}_{T}^{1}}$-module $R^{1}\left(p_{2}\right)_{*}\left(p_{1}^{*}(\mathcal{E}) \otimes \mathcal{L}\right)$ has rank $\geq 1$ (i.e., defined in terms of a fitting ideal of this sheaf).

One can prove that $\mathcal{N} \otimes \mathcal{O}\left(-2 \Theta_{T}^{1}\right)$ is isomorphic to the pullback of an invertible $\mathcal{O}_{T}$-module on $T$. Hence the divisor $\mathcal{D}_{\mathcal{E}}$ defines an $S$-morphism of $T$ into $\mathbf{P}$ :

$$
\varphi_{(T, \mathcal{E})}: T \longrightarrow \mathbf{P} \text {. }
$$

These constructions define therefore a transformation of the stack of semi-stable rank 2 bundles with trivial determinant on $\mathcal{C}$ over $S$ towards the scheme $\mathbf{P}$. It turns out that this defines an isomorphism of the coarse moduli scheme

$$
\mathcal{M}_{\mathcal{C}} \longrightarrow \mathbf{P}
$$

towards $\mathbf{P}$; see [NR].

A remark about the natural determinant bundle $\mathcal{L}$ on $\mathcal{M}_{\mathcal{C}}$ (see [Hi], page 360): The relative Picard group of $\mathbf{P}$ over $S$ is $\mathbf{Z}$ and is generated by $\mathcal{O}_{\mathbf{P}}(1)$. Hence it is clear that $\mathcal{L} \cong \mathcal{O}_{\mathbf{P}}(n) \otimes p^{*}(\mathcal{N})$ for some invertible $\mathcal{O}_{S}$-module $\mathcal{N}$ on $S$. The integer $n$ may be determined as follows. We know by [Hi], page 360 , that $\mathcal{O}_{P}(n)^{-\lambda} \cong K_{P}$. The integer $\lambda=4$ in this case, hence $n=1$. We are going to define a projective heat operator on $\mathcal{L}^{\otimes k}$ over $S$, but we have seen in Subsection 2.3.5 that this is the same as defining a projective heat operator on $\mathcal{O}_{\mathbf{P}}(k)$. Hence we will work with the line bundle $\mathcal{O}_{\mathbf{P}}(k)$ from now on. (Note that as both $\mathcal{O}_{\mathbf{P}}(1)$ and $\mathcal{L}$ are defined on the moduli stack, they must be related by a line bundle coming from the moduli stack $\mathcal{M}_{2}$ of curves of genus 2 ; however $\operatorname{Pic}\left(\mathcal{M}_{2}\right)$ is rather small.)

Let $\mathrm{Pic}^{0}=\mathrm{Pic}_{\mathcal{C} / S}^{0} \rightarrow S$ denote the Picard scheme of invertible $\mathcal{O}_{\mathcal{C}}$ bundles of relative degree 0 on $\mathcal{C}$ over $S$. There is a natural $S$-morphism

$$
f: \mathrm{Pic}^{0} \longrightarrow \mathbf{P}
$$

which in terms of the moduli-interpretations of both spaces can be defined as follows:

$$
\operatorname{Pic}^{0}(C) \ni[L] \longmapsto\left[L \oplus L^{-1}\right] \in \mathbf{P}(\mathbf{C}) .
$$

It is clear that the divisor $D_{E}$ associated to $E=L \oplus L^{-1}$ is equal to

$$
D_{E}=\left(\Theta_{C}^{1}+[L]\right) \cup\left(\Theta_{C}^{1}+\left[L^{-1}\right]\right) \subset \operatorname{Pic}^{1}(C) .
$$

This implies readily that $f^{*} \mathcal{O}_{\mathbf{P}}(1) \cong \mathcal{O}_{\mathrm{Pic}^{0}}\left(2 \Theta^{0}\right)$, in fact $f^{*} \mathcal{O}_{\mathbf{P}}(1)$ may serve as the definition of $\mathcal{O}_{\mathrm{Pic}^{0}}\left(2 \Theta^{0}\right)$ on $\mathrm{Pic}^{0}$. This induces an isomorpism $\mathbf{P} \cong\left|\mathcal{O}_{\mathrm{Pic}^{0}}\left(2 \Theta^{0}\right)\right|^{*}$ which identifies $f$ with the natural map. Thus $f$ factors over $\mathcal{K}:=\operatorname{Pic}^{0} /\langle \pm 1\rangle$, the relative Kummer surface, and defines a closed immersion (over $S$ ):

$$
\mathcal{K} \hookrightarrow \mathbf{P} .
$$


Any $E$ on $C$ that is semi-stable but not stable is an extension of the form $0 \rightarrow L \rightarrow E \rightarrow L^{-1} \rightarrow 0$, with $L$ of degree zero. This bundle is $S$-equivalent to the bundle $L \oplus L^{-1}$. Thus the open subscheme $\mathbf{P}^{s} \subset \mathbf{P}$ is equal to the complement of the image of $f: \mathrm{Pic}^{0} \rightarrow \mathbf{P}$.

2.5.2. Automorphisms. The group scheme $\mathcal{H}=\operatorname{Pic}^{0}[2]$ of 2-torsion points of $\operatorname{Pic}^{0}$ over $S$ acts on the schemes $\mathrm{Pic}^{1}$. Indeed, if $\mathcal{E}$ over $\mathcal{C}_{T}$ is a family of locally free sheaves as above and if $\mathcal{A}$ is a line bundle on $\mathcal{C}_{T}$ which defines a 2-torsion point of $\mathrm{Pic}^{0}$, then $\mathcal{E} \otimes \mathcal{A}$ is another family of locally free sheaves satisfying (a) and (b) of 2.5.1. This defines an action $\mathcal{H} \times{ }_{S} \mathbf{P} \rightarrow \mathbf{P}$ of $\mathcal{H}$ on $\mathbf{P}$, given the identification of $\mathbf{P}$ as the moduli scheme. It is easy to see that this action is induced from the natural action of $\mathcal{H}$ on $\mathrm{Pic}^{1}$. Furthermore the morphism $f: \mathrm{Pic}^{0} \rightarrow \mathbf{P}$ is equivariant with respect to this action.

Note that the action just defined does not lift to an action of $\mathcal{H}$ on $\mathcal{O}_{\mathbf{P}}(1)$. Let us write $\mathcal{G}$ for the theta group of the relatively ample line bundle $\mathcal{O}_{\mathrm{Pic}^{0}}\left(2 \Theta^{0}\right)$ on $\mathrm{Pic}^{0}$ over $S$; this group scheme gives the Heisenberg group for any point $0 \in S(\mathbf{C})$. We remark that $\mathcal{G}$ fits into the exact sequence

$$
1 \longrightarrow \mathbf{G}_{m, S} \longrightarrow \mathcal{G} \longrightarrow \mathcal{H} \longrightarrow 0 \text {. }
$$

There is a unique action of $\mathcal{G}$ on $\mathcal{O}_{\mathbf{P}}(1)$ which lifts the action of $\mathcal{H}$ on $\mathbf{P}$ and agrees with the defining action $\mathcal{G}$ on $f^{*} \mathcal{O}_{\mathbf{P}}(1)$ over $\mathrm{Pic}^{0}$.

2.5.3. Test families. Let $\mathcal{E}$ be a rank 2 free $\mathcal{O}$-module on $\mathcal{C}_{T}$ satisfying (a) and (b) of 2.5.1. We will say that the pair $(T, \mathcal{E})$ is a test family if the following conditions are satisfied:

1. $T \rightarrow S$ is smooth,

2. all $E=\mathcal{E}_{0}$ are simple vector bundles on $C=\mathcal{C}_{0}$, and

3. the map $\mathcal{T}_{T / S} \longrightarrow R^{1} \operatorname{pr}_{2 *}\left(\mathcal{E} n d_{0}(\mathcal{E})\right)$ is an isomorphism.

The last condition needs some clarification. The obstruction for the locally free sheaf $\mathcal{E}$ to have a connection on $\mathcal{C}_{T}$ is an element (the Atiyah class) in

$$
H^{1}\left(\mathcal{C} \times_{S} T, \Omega_{\mathcal{C} \times{ }_{S} T}^{1} \otimes \mathcal{E} n d(\mathcal{E})\right)
$$

We can use the maps $\Omega_{\mathcal{C} \times{ }_{S} T}^{1} \rightarrow \operatorname{pr}_{2}^{*} \Omega_{T / S}^{1}$ and $\mathcal{E} n d(\mathcal{E}) \rightarrow \mathcal{E} n d_{0}(\mathcal{E})$ to project this to a section of $\Omega_{T / S}^{1} \otimes R^{1} \mathrm{pr}_{2 *}\left(\mathcal{E} n d_{0}(\mathcal{E})\right)$, whence the map of condition 3 . This condition means that the deformation of $E=\mathcal{E}_{0}$ for any $0 \in T$ is versal in the "vertical direction".

The following lemma is proved in the usual manner, using deformation theory (no obstructions!) and Artin approximation.

2.5.4. Lemma. For any $0 \in S(\mathbf{C})$ and any simple bundle $E$ on $C=\mathcal{C}_{0}$ there exists a test family $(T, \mathcal{E})$ such that $(E, C)$ occurs as one of its fibres.

2.5.5. Lemma. Let $(T, \mathcal{E})$ be a test family. The morphism $\varphi_{(T, \mathcal{E})}: T \rightarrow \mathbf{P}$ is étale.

Proof. Let $E$ be a simple rank 2 bundle on a smooth projective genus two curve $C$ over $\operatorname{Spec}(\mathbf{C})$ with $\operatorname{det}(E)=\mathcal{O}_{C}$. Let $\eta \in H^{1}\left(C, \mathcal{E} n d_{0}(E)\right)$. For each invertible sheaf $L$ on $C$, with $\operatorname{deg}(L)=-1$ and given an embedding $L \subset E$, consider the map

$$
\mathcal{E} n d_{0}(E) \longrightarrow \mathcal{H o m}(L, E) .
$$

We have to show (*): If for all $L \subset E$ as above $\eta$ maps to 0 in $H^{1}(C, \mathcal{H} o m(L, E))$, then $\eta=0$. 
Indeed, the condition $\eta \mapsto 0$ means that the trivial deformation $L[\epsilon]$ of $L$ over $C[\epsilon]=C \times \operatorname{Spec}(\mathbf{C}[\epsilon])$ can be embedded into the deformation of $E$ given by $\eta$. If this holds for all $L \subset E$, then the divisor $D_{E}$ does not move, and we want this to imply that the infinitesimal deformation of $E$ is trivial.

Let us prove $(*)$. We will do this in the case that $E$ is not stable, as we already have the result in the stable case; see Subsection 2.4.1. Thus we may assume the bundle $E$ is simple but not stable: $E$ is a non-trivial extension

$$
0 \longrightarrow A \longrightarrow E \longrightarrow A^{-1} \longrightarrow 0
$$

with $\operatorname{deg}_{C} A=0, A^{\otimes 2} \approx \mathcal{O}_{C}$. In this case it is easy to see that there exist three line bundles $L_{1} \subset E, L_{2} \subset E$ and $L_{3} \subset E$ of degree -1 on $C$ such that the arrow towards $E$ in the following exact sequence is surjective:

$$
0 \longrightarrow K \longrightarrow L_{1} \oplus L_{2} \oplus L_{3} \longrightarrow E \longrightarrow 0
$$

Here $K$ is just the kernel of the surjection. For example we can take $L_{i}$ of the form $A^{-1}\left(-P_{i}\right)$ for $i=1,2$ and $L_{3}$ of the form $A\left(-P_{3}\right)$. (This is the only thing we will need in the rest of the argument; it should be easy to establish this in the case of a stable bundle $E$ also.) This property then holds for $L_{i} \subset E$ sufficiently general also; choose $L_{i}$ sufficiently general. Note that $K \cong L_{1} \otimes L_{2} \otimes L_{3}$ as $\operatorname{det}(E)=\mathcal{O}_{C}$.

Suppose we have a deformation $E_{\epsilon}$ of $E$ given by $\eta$ as above, i.e., we have $L_{i}[\epsilon] \rightarrow E_{\epsilon}$ lifting $L \rightarrow E$. This determines an exact sequence

$$
0 \longrightarrow K_{\epsilon} \longrightarrow L_{1}[\epsilon] \oplus L_{2}[\epsilon] \oplus L_{3}[\epsilon] \longrightarrow E_{\epsilon} \longrightarrow 0 \text {. }
$$

But $\operatorname{det} E_{\epsilon} \cong \mathcal{O}_{\mathcal{C}}[\epsilon]$, hence $K_{\epsilon} \cong L_{1}[\epsilon] \otimes L_{2}[\epsilon] \otimes L_{3}[\epsilon] \cong\left(L_{1} \otimes L_{2} \otimes L_{3}\right)[\epsilon]$ is trivial as well. Now note that by our general position we have

$$
\operatorname{dim} H^{0}\left(C, \mathcal{H} \text { om }\left(K, L_{1}\right)\right)=\operatorname{dim} H^{0}\left(C, L_{2} \otimes L_{3}\right)=1,
$$

and similarly for $L_{2}$ and $L_{3}$. Therefore, up to a unit in $\mathbf{C}[\epsilon]$, there is only one map $K[\epsilon] \rightarrow L_{i}[\epsilon]$, lifting $K \rightarrow L_{i}$. Thus the sequence (1) above is uniquely determined up to isomorphism, hence $E_{\epsilon} \cong E[\epsilon]$ is constant, i.e., $\eta=0$.

2.6. Results. This lemma completes the preparations. In the remainder of this section we prove the desired results on the existence and the characterization of the connection.

2.6.1. Theorem. The Hitchin symbol $\rho_{\text {Hitchin }}^{s}: p^{*} \mathcal{T}_{S} \rightarrow S^{2} \mathcal{T}_{\mathbf{P}^{s} / S}$ defined in Subsection 2.4.1 extends to a symbol

$$
\rho_{\text {Hitchin }}: p^{*} \mathcal{T}_{S} \longrightarrow S^{2} \mathcal{T}_{\mathbf{P} / S}
$$

Proof. Let $(T, \mathcal{E})$ be a test family. We have the morphism $\varphi=\varphi_{(T, \mathcal{E})}: T \rightarrow \mathbf{P}$ which is étale and which induces therefore an isomorphism $\mathcal{T}_{T / S} \rightarrow \varphi^{*} \mathcal{T}_{\mathbf{P} / S}$. On the other hand we have the isomorphism $\mathcal{T}_{T / S} \rightarrow R^{1} \mathrm{pr}_{2 *}\left(\mathcal{E} n d_{0}(\mathcal{E})\right)$ of the test family $(T, \mathcal{E})$. We also have the pairing as in Subsection 2.4.1

$$
\operatorname{pr}_{2 *}\left(\mathcal{E} n d_{0}(\mathcal{E}) \otimes \Omega_{\mathcal{C}_{T} / T}^{1}\right) \otimes \operatorname{pr}_{2 *}\left(\mathcal{E} n d_{0}(\mathcal{E}) \otimes \Omega_{\mathcal{C}_{T} / T}^{1}\right) \longrightarrow \operatorname{pr}_{2 *}\left(\left(\Omega_{\mathcal{C}_{T} / T}^{1}\right)^{\otimes 2}\right)
$$

Note that $\operatorname{pr}_{2 *}\left(\mathcal{E} n d_{0}(\mathcal{E}) \otimes \Omega_{\mathcal{C}_{T} / T}^{1}\right)$ is a locally free sheaf of $\mathcal{O}_{T}$-modules as $\mathcal{E}$ has simple fibres. These maps and duality give us together with the Kodaira-Spencer map a map

$$
\mathcal{T}_{S} \otimes \mathcal{O}_{T} \longrightarrow S^{2} \varphi^{*} \mathcal{T}_{\mathbf{P} / S}
$$

We remark that on the (non-empty) stable locus $T^{s}=\varphi^{-1}\left(\mathbf{P}^{s}\right)$ this map is equal to the pullback of $\rho_{\text {Hitchin }}^{s}$. 
Thus, by Lemma 2.5.5, we see that we can extend $\rho_{\text {Hitchin }}^{s}$ to any point $x \in \mathbf{P}(\mathbf{C})$ which is the image of some simple bundle. Note that a semi-stable bundle $E$, which is a non-trivial extension $0 \rightarrow L \rightarrow E \rightarrow L^{-1} \rightarrow 0$ with $\operatorname{deg}(L)=0$, is simple if and only if $L^{\otimes 2} \neq \mathcal{O}_{C}$. It follows from Lemma 2.5.4 and the above that we can extend $\rho_{\text {Hitchin }}^{s}$ to the complement of the codimension 3 locus $f\left(\mathrm{Pic}^{0}[2]\right)$, and hence by Hartog's theorem it extends.

2.6.2. Proposition. Let $f: \mathrm{Pic}^{0} \rightarrow \mathbf{P}$ be as in 2.5.1. Then $f$ is a submersion on the locus $\mathcal{Y}:=\operatorname{Pic}^{0} \backslash \operatorname{Pic}^{0}[2]$. There is a commutative diagram

$$
\begin{aligned}
& f^{*}\left(\rho_{\text {Hitchin }}\right): f^{*} p^{*} \mathcal{T}_{S} \longrightarrow f^{*} S^{2} \mathcal{T}_{\mathbf{P} / S} \\
& 4 \rho_{A b} \mid \mathcal{Y} \quad: \quad \mathcal{T}_{S} \otimes \mathcal{O}_{\mathcal{Y}} \longrightarrow S^{2} \mathcal{T}_{\mathcal{Y} / S}
\end{aligned}
$$

where $\rho_{A b}$ is the symbol of the abelian heat operator on $\mathcal{O}_{\mathrm{Pic}^{0}}\left(2 \Theta^{0}\right)=f^{*} \mathcal{O}_{\mathbf{P}}(1)$.

Proof. Consider once again a simple bundle $E$, which is given as an extension $0 \rightarrow L \rightarrow E \rightarrow L^{-1} \rightarrow 0$ with $\operatorname{deg}(L)=0$. Clearly, the kernel of the map

$$
H^{1}\left(C, \mathcal{E} n d_{0}(E)\right) \longrightarrow H^{1}\left(C, \mathcal{H o m}\left(L, L^{-1}\right)\right)
$$

represents deformations preserving the filtration on $E$, i.e., tangent vectors along the Kummer surface. Dually, this corresponds to the image of the map

$$
H^{0}\left(C, \mathcal{H} \text { om }\left(L^{-1}, L\right) \otimes \Omega_{C}^{1}\right) \longrightarrow H^{0}\left(C, \mathcal{E} n d_{0}(E) \otimes \Omega_{C}^{1}\right) .
$$

We use the Trace-form to identify $\mathcal{E} n d_{0}(E)$ with its dual. Note that there is a canonical exact sequence

$$
0 \longrightarrow \mathcal{O}_{C} \longrightarrow \mathcal{E} n d_{0}(E) / L^{\otimes 2} \longrightarrow L^{\otimes-2} \longrightarrow 0
$$

induced by the filtration $L \subset E$ on $E$. Note that

$$
\operatorname{dim} H^{0}\left(C,\left(\mathcal{E} n d_{0}(E) / L^{\otimes 2}\right) \otimes \Omega_{C}^{1}\right)=2,
$$

since if it were $\geq 3$, then $\operatorname{dim} H^{0}\left(C, \mathcal{E} n d_{0}(E) \otimes \Omega^{1}\right) \geq 4$, contrary to the assumption that $E$ is simple. Thus we get the exact sequence

$$
\begin{aligned}
H^{0}\left(C, \Omega^{1}\right) & \cong H^{0}\left(C,\left(\mathcal{E} n d_{0}(E) / L^{\otimes 2}\right) \otimes \Omega_{C}^{1}\right) \\
& \lll H^{0}\left(C, \mathcal{E} n d_{0}(E) \otimes \Omega_{C}^{1}\right) \hookleftarrow H^{0}\left(C, L^{\otimes 2} \otimes \Omega_{C}^{1}\right) \cong \mathbf{C} .
\end{aligned}
$$

This is (canonically) dual to the sequence of cotangent spaces

$$
0 \longrightarrow T_{[L]} \mathrm{Pic}^{0} \longrightarrow T_{f([L])}(\mathbf{P}) \longrightarrow\left(N_{\mathcal{Y}} \mathbf{P}\right)_{[L]} \longrightarrow 0 .
$$

We want to see that our symbols in the point $[L]$ lie in the space $S^{2} T_{[L]} \operatorname{Pic}^{0}$.

We have to study the map

$$
\begin{array}{rlr}
H^{0}\left(C, \mathcal{E} n d_{0}(E) \otimes \Omega_{C}^{1}\right) \times H^{0}\left(C, \mathcal{E} n d_{0}(E) \otimes \Omega_{C}^{1}\right) & \longrightarrow & H^{0}\left(C,\left(\Omega_{C}^{1}\right)^{\otimes 2}\right) \\
X \otimes \eta \times Y \otimes \omega & \longmapsto & \operatorname{Trace}(X Y) \otimes \eta \omega .
\end{array}
$$

But the elements of $H^{0}\left(C, L^{\otimes 2} \otimes \Omega_{C}^{1}\right)$, resp. those of $H^{0}\left(C, \Omega_{C}^{1}\right)$, locally look like

$$
\left(\begin{array}{ll}
0 & * \\
0 & 0
\end{array}\right) \otimes \eta, \quad \text { resp. } \quad\left(\begin{array}{cc}
1 & 0 \\
0 & -1
\end{array}\right) \otimes \omega .
$$


Therefore the pairing just reduces to twice the multiplication pairing $H^{0}\left(C, \Omega_{C}^{1}\right) \times$ $H^{0}\left(C, \Omega_{C}^{1}\right) \rightarrow H^{0}\left(C,\left(\Omega_{C}^{1}\right)^{\otimes 2}\right)$.

This multiplication pairing, however, corresponds exactly (via Kodaira-Spencer) to the symbol of the heat operator on the theta-divisor of the Jacobian $\mathrm{Pic}^{0}$ of $\mathcal{C}$ over $S$. Since we are looking at $2 \Theta^{0}$, we get the desired factor 4 ; compare with Subsections 2.3.8 and 2.3.7.

2.6.3. Corollary. The symbol $\rho_{\text {Hitchin }}$ is invariant under the action of the group scheme $\mathcal{H}=\operatorname{Pic}^{0}[2]$ on $\mathbf{P}$ and $S^{2} \mathcal{T}_{\mathbf{P} / S}$.

Proof. This follows from the observation that it is true for $\rho_{A b \mid \mathcal{Y}}$ and thus for $\left.\rho_{\text {Hitchin }}\right|_{\mathcal{K}}$, the Hitchin symbol restricted to the Kummer surface. Further, one uses the remark that there are no non-vanishing elements of $H^{0}\left(\mathbf{P}, S^{2} \mathcal{T}_{\mathbf{P} / S}\right)$ which vanish on $\mathcal{K}$, that is, $H^{0}\left(\mathbf{P}, S^{2} \mathcal{T}_{\mathbf{P} / S}(-4)\right)=0$.

2.6.4. Proposition. There exists a unique projective heat operator $\bar{D}_{\lambda, k}$ (with $\lambda \in$ $\mathbf{C}^{*}$ a nonzero complex number) on $\mathcal{O}_{\mathbf{P}}(k)$ over $S$ with the following properties:

1. the symbol of $\bar{D}_{\lambda, k}$ is equal to $\frac{1}{4 \lambda} \rho_{\text {Hitchin }}$, and

2. the operator $\bar{D}_{\lambda, k}$ commutes (projectively) with the action of $\mathcal{G}$ on $\mathcal{O}_{\mathbf{P}}(k)$.

Proof. This follows readily from the discussion in Subsection 2.3.7. Indeed, both obstructions mentioned there vanish in view of the vanishing of $R^{1} p_{*} \mathcal{I}_{\mathbf{P} / S}$ and $R^{1} p_{*} \mathcal{O}_{\mathbf{P}}$. We have a good way of choosing the elements $D(\theta)$ : namely, we choose them $\mathcal{G}$-invariantly. This is possible: first choose arbitrary lifts, and then average over liftings of a full set of sections of $\mathcal{H}$ (this can be done étale locally over $S$ ). This determines the $D(\theta)$ uniquely up to an element of $p^{-1}\left(\mathcal{O}_{S}\right)$, as $\left(p_{*} \mathcal{I}_{\mathbf{P} / S}\right)^{\mathcal{H}}=(0)$. Hence we get the desired projective heat operator.

It follows from Proposition 2.6.2 that the heat operators $\bar{D}_{\lambda, k}$ are compatible with the morphism $f: \mathcal{Y} \rightarrow \mathbf{P}$. Also, for $k=1$ and $\lambda=1$, we see that $\bar{D}_{1,1}$ is compatible with the abelian heat operator on $\mathcal{O}_{\mathrm{Pic}^{0}}\left(2 \Theta^{0}\right)$ restricted to $\mathcal{Y}$ (this is one of the reasons for the factor $\frac{1}{4}$, but see below also).

2.6.5. Hitchin's connection for genus 2 and rank 2. The title of this subsection is somewhat misleading. As mentioned before, in the paper [Hi] there is no definition of a heat operator in the case of genus 2 and rank 2. However, we propose the following definition.

2.6.6. Definition. The (projective) Hitchin connection on $p_{*} \mathcal{O}_{\mathbf{P}}(k)$ is the projective connection associated to the projective heat operator $\mathbf{D}_{k}:=\bar{D}_{(k+2), k}$.

This definition makes sense for the following reason: The symbol of the operator $\bar{D}_{(k+2), k}$ is equal to $2 /(2 k+4) \rho_{\text {Hitchin }}$ which is equal to the symbol that occurs in [Hi], Theorem 3.6 (the factor 2 comes from our way of normalizing symbols; see Subsection 2.2.4). Thus the only assumption we needed in order to get $\mathbf{D}_{k}$ was the assumption that it is compatible with the action of $\mathcal{G}$; see Subsection 2.6.4.

2.6.7. How to compute the heat operators? To determine $\mathbf{D}_{k}$ we introduce heat operators $D_{\lambda, k}$ under the following assumptions:

1. The family of Kummer surfaces $\mathcal{K} \subset \mathbf{P}$ is given by one equation

$$
F \in \Gamma\left(\mathbf{P}, \mathcal{O}_{\mathbf{P}}(4)\right) .
$$


2. We have chosen an integrable connection $\nabla_{0}$ on $\mathcal{F}:=p_{*} \mathcal{O}_{\mathbf{P}}(1)$ over $S$ which is equivariant for the action of $\mathcal{G}$.

We remark that both conditions can be satisfied on the members of an open covering of $S$. Also the connection $\nabla_{0}$ in 2 is determined uniquely up to the addition of a linear operator of the form $\eta$. id for some closed 1-form $\eta$ on $S$. We remark that $\nabla_{0}$ induces a connection $\nabla_{0}$ on all the locally free sheaves $S^{k} \mathcal{F}=p_{*} \mathcal{O}_{\mathbf{P}}(k)$ over $S$, but these should not be confused with the connections induced by the $D_{\lambda, k}$ ! In addition $\nabla_{0}$ determines an integrable connection on $\mathbf{P}$ over $S$ and a lift of this to connections on the sheaves $\mathcal{O}_{\mathbf{P}}(k)$ over $\mathbf{P}$. In other words we have rigidified $\left(\mathbf{P}, \mathcal{O}_{\mathbf{P}}(k)\right)$ over $S$.

There is a natural surjection

$$
S^{2} \mathcal{F} \otimes_{\mathcal{O}_{S}} S^{2} \mathcal{F}^{*} \longrightarrow p_{*} S^{2} \mathcal{T}_{\mathbf{P} / S}
$$

which has a canonical splitting, given by decomposing $S^{2} \mathcal{F} \otimes \mathcal{O}_{S} S^{2} \mathcal{F}^{*}$ into irreducible $\mathrm{GL}(\mathcal{F})$-modules, i.e., the unique $\operatorname{GL}(\mathcal{F})$-equivariant splitting. Thus we identify a section $X$ of $S^{2} \mathcal{T}_{\mathbf{P} / S}$ with a section $X$ of $S^{2} \mathcal{F} \otimes_{\mathcal{O}_{S}} S^{2} \mathcal{F}^{*}$. Note that we can regard sections of $S^{2} \mathcal{F} \otimes_{\mathcal{O}_{S}} S^{2} \mathcal{F}^{*}$ as sections of $\operatorname{Diff}_{\mathbf{P} / S}^{(2)}\left(\mathcal{O}_{\mathbf{P}}(k)\right)$ in a natural manner, by considering them as second order differential operators on the affine 4 -space $\operatorname{Spec}\left(S^{*} \mathcal{F}\right)$ invariant under the scalar action of $\mathbf{G}_{m, S}$. We write $E$ for the Euler-vector field, i.e., the section of $\mathcal{F} \otimes \mathcal{F}^{*}$ that corresponds to the identity map of $\mathcal{F}$.

Assume we have a $\mathcal{G}$-invariant section

$$
X \in S^{2} \mathcal{F} \otimes_{\mathcal{O}_{S}} S^{2} \mathcal{F}^{*} \otimes_{\mathcal{O}_{S}} \Omega_{S}^{1}
$$

with the property

$$
X \cdot F=\nabla_{0}(F) E \quad \text { in } \quad S^{5}(\mathcal{F}) \otimes_{\mathcal{O}_{S}} \mathcal{F}^{*} \otimes_{\mathcal{O}_{S}} \Omega_{S}^{1},
$$

where $X \cdot F$ denotes contracting once (note that $F$ can be viewed as a section of $\left.S^{4} \mathcal{F}\right)$.

If we have such an $X$ we define a heat operator

$$
D_{\lambda, k}: p^{*} \mathcal{T}_{S} \longrightarrow \operatorname{Diff}_{\mathbf{P}}^{(2)}\left(\mathcal{O}_{\mathbf{P}}(k)\right)
$$

by the formula $\left(\theta\right.$ a local section of $\left.\mathcal{T}_{S}\right)$

$$
D_{\lambda, k}(\theta)=\theta-\frac{1}{\lambda} X_{\theta, k}
$$

Here $X_{\theta, k}$ means the following: first contract $X$ with $\theta$ to get a section $X_{\theta}$ of $S^{2} \mathcal{F} \otimes_{\mathcal{O}_{S}} S^{2} \mathcal{F}^{*}$ and then consider this as a second order differential operator $X_{\theta, k}$ of $\mathcal{O}_{\mathbf{P}}(k)$ on $X$ over $S$ by the remarks above. Note that the term $\theta$ acts on $\mathcal{O}_{\mathbf{P}}(k)$ using $\nabla_{0}$.

2.6.8. Lemma. Let $D_{\lambda, k}$ be the heat operator defined above.

1. The heat operator $D_{\lambda, k}$ commutes with the action of $\mathcal{G}$ on $\mathcal{O}_{\mathbf{P}}(k)$.

2. The heat operators $D_{\lambda, k}$ are compatible with $f: \mathcal{Y} \rightarrow \mathbf{P}$. If $\lambda=k$, then $D_{k, k}$ is strictly compatible with $f: \mathcal{Y} \rightarrow \mathbf{P}$.

Proof. The second statement holds since $X$ is $\mathcal{G}$-invariant. We now verify the last statement. Note that $D_{k, k}$ is strictly compatible with $\mathcal{K} \rightarrow \mathbf{P}$ if the operators $D_{k, k}(\theta)$ preserve the subsheaf $\mathcal{I}_{\mathcal{K}} \cdot \mathcal{O}_{\mathbf{P}}(k)$ of $\mathcal{O}_{\mathbf{P}}(k)$. This can be seen by the description of strict compatiblility in terms of local coordinates given in Subsection 
2.3.10. We may check this condition on the affine 4-space $\operatorname{Spec}\left(S^{*} \mathcal{F}\right)$. Thus let $G \in S^{k-4}(\mathcal{F})$ be a section, let $\theta$ be a local vector field on $S$ and consider

$$
\nabla_{0, \theta}(G F)-\frac{1}{k} X_{\theta}(G F)
$$

We have to show that this is divisible by $F$.

We may assume that $G$ is horizontal for $\nabla_{0}$, as we can find a horizontal basis for $S^{k-4}(\mathcal{F})$ locally. Thus we get

$$
G \nabla_{0, \theta}(F)-\frac{1}{k} X_{\theta}(G F)=G \nabla_{0, \theta}(F)-\frac{1}{k} X_{\theta}(G) F-\frac{1}{k}(X \cdot F)_{\theta}(G)-\frac{1}{k} G X_{\theta}(F) .
$$

Here we have used a general formula for the application of a second order operator like $X_{\theta}$ on a product like $F G$. Note also that $X(F)$ is simply the contraction of $X \cdot F$, hence by $(E q$.$) we get$

$$
G \nabla_{0, \theta}(F)-\frac{1}{k} X_{\theta}(G) F-\frac{1}{k} \nabla_{0, \theta}(F) E(G)-\frac{1}{k} G E\left(\nabla_{0, \theta}(F)\right) .
$$

Next, we use that acting by $E$ on a homogeneous polynomial gives degree times the polynomial:

$$
G \nabla_{0, \theta}(F)-\frac{1}{k} X_{\theta}(G) F-\frac{1}{k} \nabla_{0, \theta}(F)(k-4) G-\frac{1}{k} G 4 \nabla_{0, \theta}(F)=-\frac{1}{k} X_{\theta}(G) F
$$

which is divisible by $F$. Thus $D_{k, k}$ is indeed strictly compatible with $f: \mathcal{Y} \rightarrow \mathbf{P}$.

2.6.9. Theorem. Let $X$ be a solution to the equation (Eq.) above and let $D_{\lambda, k}$ be the heat operators defined in this subsection. Then

1. The projective heat operator defined by $D_{\lambda, k}$ is the operator $\bar{D}_{\lambda, k}$ defined in Subsection 2.6.4. Thus $D_{k+2, k}$ defines the Hitchin connection on $\mathcal{O}_{\mathbf{P}}(k)$.

2. The heat operators $D_{\lambda, k}$ are projectively flat for any $k$ and $\lambda$.

3. For $\lambda=k$, the operator $D_{k, k}$ is strictly compatible with the abelian projective heat operator on $\mathcal{O}_{\mathrm{Pic}^{0}}\left(\mathcal{O}\left(2 k \Theta^{0}\right)\right)$ over an open part of $\mathrm{Pic}^{0}$.

Proof. Consider first the case of $D_{k, k}$ as defined in 2.6.7. We have seen above that it is strictly compatible with $f: \mathcal{Y} \rightarrow \mathbf{P}$. Therefore, by Subsection 2.3.10 it defines a heat operator $D_{k}$ on $f^{*} \mathcal{O}_{\mathbf{P}}(k)$ over $\mathcal{Y}$. In view of Hartog's theorem this extends uniquely to a heat operator $D_{k}$ of $\mathcal{O}_{\mathrm{Pic}^{0}}\left(\mathcal{O}\left(2 k \Theta^{0}\right)\right)$ on $\mathrm{Pic}^{0}$ over $S$. We have seen in Subsection 2.3.8 that there is a unique such heat operator, whose symbol is $1 / k$ times the symbol $\rho_{A b}$ of $D_{1}$. Combining this with the assertions in Subsection 2.6.2 we see that $(1 / 4 k) \rho_{\text {Hitchin }}-\rho_{D_{k, k}}$ vanishes along $\mathcal{K}$ and hence is zero. This proves that $D_{k, k}$ agrees with the projective heat operator $\bar{D}_{k, k}$ defined in Subsection 2.6.4.

Now it follows from the transformation behaviour of the symbol of $\bar{D}_{\lambda, k}$ and $D_{\lambda, k}$ that these agree for arbitrary $\lambda$. Thus we get the agreement stated in the theorem.

To see that these heat operators are projectively flat, we argue as follows. Let $\theta, \theta^{\prime}$ be two local commuting vector fields on $S$. We have to see that the section $\left[D_{\lambda, k}(\theta), D_{\lambda, k}\left(\theta^{\prime}\right)\right]$ of $\operatorname{Difff}_{\mathbf{P} / S}^{(3)}\left(\mathcal{O}_{\mathbf{P}}(k)\right)$ lies in the subsheaf $p^{-1}\left(\mathcal{O}_{S}\right)$. Again, for $\lambda=k$ this section is zero when restricted to $\mathcal{K}$, and again this implies that the section is zero in that case. Now, let us compute

$$
\begin{aligned}
{\left[D_{\lambda, k}(\theta), D_{\lambda, k}\left(\theta^{\prime}\right)\right] } & =\left[\theta-\frac{1}{\lambda} X_{\theta, k}, \theta^{\prime}-\frac{1}{\lambda} X_{\theta^{\prime}, k}\right] \\
& =\frac{1}{\lambda}\left(\left[\theta, X_{\theta^{\prime}, k}\right]-\left[\theta^{\prime}, X_{\theta, k}\right]\right)+\frac{1}{\lambda^{2}}\left[X_{\theta, k}, X_{\theta^{\prime}, k}\right]
\end{aligned}
$$


The index ${ }_{k}$ is now superfluous, as we can see this as an expression in $S^{2} \mathcal{F} \otimes_{\mathcal{O}_{S}}$ $S^{2} \mathcal{F}^{*} \oplus S^{3} \mathcal{F} \otimes_{\mathcal{O}_{S}} S^{3} \mathcal{F}^{*}$. We know that this expression is zero for any $k$ with $\lambda=k$ considered as a third order operator on $\mathcal{O}_{\mathbf{P}}(k)$. This implies that both terms are zero, hence the expression is zero for any $\lambda$ and any $k$. (We remark for the doubtful reader that we will verify the flatness also by a direct computation using the explicit description of the operator.)

\section{The flat CONNECTION}

3.1. We introduce certain families of flat connections on (trivial) bundles over the configuration space $\mathcal{P}$ (cf. 1.4). These are defined by representations of the Lie algebra $s o(2 g+2)$. Then we derive a convenient form for the equation of the family of Kummer surfaces $\mathcal{K} \hookrightarrow \mathbf{P}$ (in fact for any $g$ we point out a specific section $P_{z}$ of a trivial bundle over $\mathcal{P}$ ). In Theorem 3.4.3 we show that this equation is flat for one of our connections. This will be important in identifying Hitchin's connection in the next section.

\subsection{Orthogonal groups.}

3.2.1. The Lie algebra. Let $Q=x_{1}^{2}+\ldots+x_{2 g+2}^{2}$. Then the (complex) Lie algebra $s o(Q)=s o(2 g+2)$ is:

$$
s o(2 g+2)=\left\{A \in \operatorname{End}\left(\mathbf{C}^{2 g+2}\right):{ }^{t} A+A=0\right\} ;
$$

let $F_{i j}:=2\left(E_{i j}-E_{j i}\right)(\in s o(2 g+2))$, where $E_{i j}$ is the matrix whose only non-zero entry is a 1 in the $(i, j)$-th position (the commutator of such matrices is $\left[E_{i j}, E_{k l}\right]=$ $\delta_{j k} E_{i l}-\delta_{l i} E_{k j}$ ). The alternating matrices $F_{i j}$ (with $1 \leq i<j \leq 2 g+2$ ) are a basis of $s o(2 g+2)$. Note $F_{i j}=-F_{j i}$. These matrices satisfy the relations:

$$
\left[F_{i j}, F_{k l}\right]=\left\{\begin{array}{rll}
0 & \text { if } \quad i, j, k, l \text { are distinct, } \\
2 F_{i l} & \text { if } \quad j=k .
\end{array}\right.
$$

3.2.2. The universal enveloping algebra. Recall that for a Lie algebra $\mathbf{g}$ the tensor algebra $T(\mathbf{g})$ and the universal enveloping algebra $U(\mathbf{g})$ are defined by:

$$
T(\mathbf{g}):=\mathbf{C} \oplus \mathbf{g} \oplus \mathbf{g} \otimes \mathbf{g} \ldots, \quad U(\mathbf{g}):=T(\mathbf{g}) / I
$$

where $I$ is the ideal generated by all elements of the form $x \otimes y-y \otimes x-[x, y]$ with $x, y \in \mathbf{g}$. Lie algebra representations $\rho: \mathbf{g} \rightarrow \operatorname{End}(V)($ so $\rho([x, y])=\rho(x) \rho(y)-$ $\rho(y) \rho(x))$ correspond to representations $\tilde{\rho}: U(\mathbf{g}) \rightarrow \operatorname{End}(V)$ of associative algebras with $\rho(1)=\operatorname{id}_{V}$. Given $\rho$ one defines $\tilde{\rho}\left(x_{i_{1}} \otimes x_{i_{2}} \otimes \ldots \otimes x_{i_{k}}\right):=\rho\left(x_{i_{1}}\right) \rho\left(x_{i_{2}}\right) \ldots \rho\left(x_{i_{k}}\right)$ which is well-defined because we work modulo $I$.

3.2.3. Definitions. We define elements in $U(s o(2 g+2))$ by:

$$
\Omega_{i j}:=F_{i j} \otimes F_{i j} \quad \bmod I \quad(\in U(s o(2 g+2))), \quad \text { note } \quad \Omega_{i j}=\Omega_{j i} .
$$

In particular, for any Lie algebra representation $\rho: s o(2 g+2) \rightarrow W$ we now have endomorphisms $\tilde{\rho}\left(\Omega_{i j}\right)=\rho\left(F_{i j}\right)^{2}: W \rightarrow W$ which satisfy the same commutation relations as the $\Omega_{i j}$.

For $\lambda \in \mathbf{C}^{*}$ we define a $U(s o(2 g+2))$-valued one-form $\omega_{\lambda}$ on $\mathcal{P}$ :

$$
\omega_{\lambda}:=\lambda^{-1}\left(\sum_{i \neq j} \frac{\Omega_{i j} \mathrm{~d} z_{i}}{z_{i}-z_{j}}\right) ; \quad \text { let } \quad \tilde{\rho}\left(\omega_{\lambda}\right):=\lambda^{-1}\left(\sum_{i \neq j} \frac{\tilde{\rho}\left(\Omega_{i j}\right) \mathrm{d} z_{i}}{z_{i}-z_{j}}\right)
$$


where $\tilde{\rho}: U(s o(2 g+2)) \rightarrow \operatorname{End}(W)$ is a representation. Note $\tilde{\rho}\left(\omega_{\lambda}\right) \in \operatorname{End}(W) \otimes \Omega_{\mathcal{P}}^{1}$.

The one-form $\omega_{\lambda}$ defines a connection on the trivial bundle $W \otimes_{\mathbf{C}} \mathcal{O}_{\mathcal{P}}$ by:

$\nabla_{\lambda}^{W}: W \otimes \mathcal{O}_{\mathcal{P}} \longrightarrow W \otimes \Omega_{\mathcal{P}}^{1}, \quad f w \longmapsto w \otimes \mathrm{d} f-f \tilde{\rho}\left(\omega_{\lambda}\right)(w) \quad\left(f \in \mathcal{O}_{\mathcal{P}}, w \in W\right)$.

The covariant derivative of $f \otimes w$ with respect to the vector field $\partial / \partial z_{i}$ on $\mathcal{P}$ is the composition of $\nabla_{\lambda}^{W}$ with contraction:

$$
\left(\nabla_{\lambda}^{W}(f \otimes w)\right)_{\partial / \partial z_{i}}=w \otimes \frac{\partial f}{\partial z_{i}}-\sum_{j, j \neq i} \tilde{\rho}\left(\Omega_{i j}\right)(w) \otimes \frac{1}{z_{i}-z_{j}} \quad\left(\in W \otimes \mathcal{O}_{\mathcal{P}}\right) .
$$

3.2.4. Proposition. Let $\rho: s o(2 g+2) \longrightarrow \operatorname{End}(W)$ be a Lie algebra representation. Then $\nabla_{\lambda}^{W}$ is a flat connection on $W \otimes \mathcal{O}_{\mathcal{P}}$ for any $\lambda \in \mathbf{C}^{*}$.

Proof. Obviously $\nabla_{\lambda}^{W}$ is a connection. It is well known (and easy to verify) that the connection defined by $\omega$ is flat (so $\mathrm{d} \omega+\omega \wedge \omega=0$ ) if the following infinitesimal pure braid relations in $U(s o(2 g+2))$ are satisfied:

$$
\left[\Omega_{i j}, \Omega_{k l}\right]=0, \quad\left[\Omega_{i k}, \Omega_{i j}+\Omega_{j k}\right]=0,
$$

where $i, j, k, l$ are distinct indices (cf. [Ka], Section XIX.2).

To check these relations we use that $X \otimes Y=Y \otimes X+[X, Y]$ in $U(s o)$. The first relation is then obviously satisfied. We spell out the second. Consider first:

$$
\begin{aligned}
F_{i k} \otimes F_{i j} \otimes F_{i j} & =\left(F_{i j} \otimes F_{i k}+\left[F_{i k}, F_{i j}\right]\right) \otimes F_{i j} \\
& =F_{i j} \otimes F_{i k} \otimes F_{i j}-F_{k j} \otimes F_{i j} \\
& =F_{i j} \otimes\left(F_{i j} \otimes F_{i k}-F_{k j}\right)+F_{j k} \otimes F_{i j} \\
& =F_{i j} \otimes F_{i j} \otimes F_{i k}+\left(F_{i j} \otimes F_{j k}+F_{j k} \otimes F_{i j}\right),
\end{aligned}
$$

so we have $\left[F_{i k}, F_{i j} \otimes F_{i j}\right]=D_{1}$ with $D_{1}$ symmetric in the indices $i$ and $k$. Similarly we get:

$$
\begin{aligned}
F_{i k} \otimes\left(F_{i k} \otimes F_{i j} \otimes F_{i j}\right) & =F_{i k} \otimes\left(F_{i j} \otimes F_{i j} \otimes F_{i k}+D_{1}\right) \\
& =\left(F_{i k} \otimes F_{i j} \otimes F_{i j}\right) \otimes F_{i k}+F_{i k} \otimes D_{1} \\
& =F_{i j} \otimes F_{i j} \otimes F_{i k} \otimes F_{i k}+\left(D_{1} \otimes F_{i k}+F_{i k} \otimes D_{1}\right) .
\end{aligned}
$$

Thus $\left[\Omega_{i k}, \Omega_{i j}\right]=D_{1} \otimes F_{i k}+F_{i k} \otimes D_{1}$ which is antisymmetric in $i$ and $k$. Therefore

$$
\left[\Omega_{i k}, \Omega_{i j}\right]=-\left[\Omega_{k i}, \Omega_{k j}\right]=-\left[\Omega_{i k}, \Omega_{j k}\right]
$$

which proves the second infinitesimal braid relation.

3.2.5. Differential operators. Given a Lie algebra representation $\rho: \mathbf{g} \rightarrow \operatorname{End}\left(S_{1}\right)$ where $S_{k} \subset S:=\mathbf{C}\left[\ldots, X_{i}, \ldots\right]$ is the subspace of homogeneous polynomials of degree $k$, there is a convenient way to determine the representation $\rho^{(k)}: g \rightarrow$ $\operatorname{End}\left(S_{k}\right)$ induced by $\rho$. If $\rho(A)=\left(a_{i j}\right)$ w.r.t. the basis $X_{i}$ of $S_{1}$, then we define

$$
L_{A}:=\sum_{i, j} a_{i j} X_{i} \partial_{j} \quad(\in \operatorname{End}(S)), \quad \text { with } \quad \partial_{j}(P):=\frac{\partial P}{\partial X_{j}}
$$

for $P \in S$. Then obviously $\rho(A)(P)=L_{A}(P)$ if $P$ is linear and the Leibnitz rule shows that $\rho^{(k)}(A)=L_{A}: S_{k} \rightarrow S_{k}$ for all $k$.

The composition (in $\operatorname{End}(S)$ ) of two operators is given by:

$$
\left(X_{i} \partial_{j}\right) \circ\left(X_{k} \partial_{l}\right)=X_{i} X_{k} \partial_{j} \partial_{l}+\delta_{j k} X_{i} \partial_{l}, \quad \text { thus } L_{A}^{2}=(1 / 2) \sigma\left(L_{A}^{2}\right)+L_{A^{2}},
$$


with symbol $\sigma\left(L_{A}^{2}\right)=2 \sum_{i, j, k, l} a_{i j} a_{k l} X_{i} X_{k} \partial_{j} \partial_{l}$ (see our convention 2.2.4).

Assume that we have $A_{i j} \in \mathbf{g}$ such that $\Omega_{i j}=A_{i j} \otimes A_{i j}$ satisfy the infinitesimal braid relations (cf. proof of the previous proposition) and that in the representation $\rho$ we have $\rho\left(A_{i j}\right)^{2}=\mu I$ with $\mu \in \mathbf{C}$. Then we conclude that the operators

$$
\sigma\left(L_{A_{i j}}^{2}\right): S \longrightarrow S
$$

also satisfy the infinitesimal braid relations.

\subsection{The Kummer equation.}

3.3.1. Introduction. We recall how one can obtain the equation of the Kummer variety for a genus two curve, and more generally, how a hyperelliptic curve $\mathcal{C}_{z}$ determines in a natural way a quartic polynomial $P_{z} \in S^{4} V\left(\omega_{g+1}\right)$ where $V\left(\omega_{g+1}\right)$ is a half spin representation of $s o(2 g+2)$.

The polynomial $P_{z}$ lies in the subrepresentation of $V\left(4 \omega_{g+1}\right)$ which also occurs in $S^{2}\left(\wedge^{g+1} \mathbf{C}^{2 g+2}\right)$ where $\mathbf{C}^{2 g+2}$ is the standard representation of $s o(2 g+2)$. We will exploit this fact to verify that $P_{z}$ is a flat section for one of the connections introduced in Subsection 3.2.

3.3.2. The orthogonal Grassmannian. We define two quadratic forms on $\mathbf{C}^{2 g+2}$ by:

$$
Q: \quad x_{1}^{2}+\ldots+x_{2 g+2}^{2}, \quad Q_{z}: \quad z_{1} x_{1}^{2}+\cdots+z_{2 g+2} x_{2 g+2}^{2},
$$

and we use the same symbols to denote the corresponding quadrics in $\mathbf{P}^{2 g+1}$. The quadric $Q$ has two rulings (families of linear $\mathbf{P}^{g}$ 's lying on it). Each of these is parametrized by the orthogonal Grassmannian (spinor variety) denoted by $G r_{S O}$. The variety $G r_{S O}$ is smooth and projective of dimension $\frac{1}{2} g(g+1)$.

Let $V\left(\omega_{g+1}\right)$ be a half spin representation of $s o(2 g+2)$. There is an embedding

$$
\phi: G r_{S O} \longrightarrow \mathbf{P} V\left(\omega_{g+1}\right)^{*} ; \quad \text { let } \mathcal{O}_{G r_{S O}}(1):=\phi^{*} \mathcal{O}_{\mathbf{P} V}(1) \text {. }
$$

In fact, $\phi\left(G r_{S O}\right)$ is the orbit of the highest weight vector. The map $\phi$ is equivariant for the action of the spin group $\widetilde{S O}$ (which acts through a half spin representation on $\left.V\left(\omega_{g+1}\right)\right)$. It induces isomorphisms:

$$
H^{0}\left(G r_{S O}, \mathcal{O}(n)\right)=V\left(n \omega_{g+1}\right) .
$$

In case $g=2$, one has isomorphisms:

$$
\phi: G r_{S O} \stackrel{\cong}{\longrightarrow} \mathbf{P}^{3}, \quad S_{k}=V\left(k \omega_{g+1}\right) \quad \text { and } \quad \widetilde{S O_{6}} \cong S L_{4}(\mathbf{C})
$$

and the half spin representation is identified with the standard representation $S L_{4}(\mathbf{C})$ on $\mathbf{C}^{4}$ (or its dual).

3.3.3. The Plücker map. Let $G r_{S L}$ be the Grassmannian of $g$-dimensional subspaces of $\mathbf{P}^{2 g+1}$. We denote the Plücker map by:

$$
\begin{gathered}
p: G r_{S L} \longrightarrow \mathbf{P} \wedge{ }^{g+1} \mathbf{C}^{2 g+2}, \\
\left\langle v_{1}, \ldots, v_{g+1}\right\rangle \longmapsto v_{1} \wedge \ldots \wedge v_{g+1}=\sum p_{i_{1} \ldots i_{g+1}} e_{i_{1}} \wedge \ldots \wedge e_{i_{g+1}} .
\end{gathered}
$$

The map $p$ is equivariant for the $S L(2 g+2)$-action on both sides and induces isomorphisms of $\operatorname{sl}(2 g+2)$-representations

$$
H^{0}\left(G r_{S L}, \mathcal{O}_{G r_{S L}}(n)\right)=V\left(n \lambda_{g+1}\right), \quad \text { with } \quad V\left(\lambda_{g+1}\right) \cong \wedge^{g+1} \mathbf{C}^{2 g+2} .
$$


As a representation of $s o(2 g+2)$ the space $\wedge^{g+1} \mathbf{C}^{2 g+2}$ is reducible; one has (cf. [vG], (3.7), [FH], §19.2, Th. 19.2 and Remarks (ii)), with $V\left(\omega_{g}\right)$ and $V\left(\omega_{g+1}\right)$ the half spin representations:

$$
\wedge^{g+1} \mathbf{C}^{2 g+2}=V\left(2 \omega_{g}\right) \oplus V\left(2 \omega_{g+1}\right) .
$$

In particular, the irreducible component $V\left(4 \omega_{g+1}\right)$ of $S^{4} V\left(\omega_{g+1}\right)$ is also a component of $S^{2}\left(\wedge^{g+1} \mathbf{C}^{2 g+2}\right)$ (viewed as an $s o(2 g+2)$ representation). Elements of $S^{2}\left(\wedge^{g+1} \mathbf{C}^{2 g+2}\right)$ can be seen as quadratic forms in the Plücker coordinates, when restricted to $G r_{S O}$ they can be viewed as (restrictions to $G r_{S O}$ of) quartic polynomials on $\mathbf{P} V\left(\omega_{g+1}\right)^{*}$.

3.3.4. Notation. The following lemma gives this decomposition of $\wedge^{g+1} \mathbf{C}^{2 g+2}$ explicitly. Let $\left\{e_{i}\right\}$ be the standard basis of $\mathbf{C}^{2 g+2}$. For any

$$
S \subset B:=\{1,2, \ldots, 2 g+2\}, \quad|S|=g+1,
$$

we write $S=\left\{i_{1}, i_{2}, \ldots, i_{g+1}\right\}$ with $i_{1}<i_{2}<\ldots<i_{g+1}$ and define

$$
e_{S}:=e_{i_{1}} \wedge \ldots \wedge e_{i_{g+1}} \in \wedge^{g+1} \mathbf{C}^{2 g+2} .
$$

These elements give a basis of $\wedge^{g+1} \mathbf{C}^{2 g+2}$. For such an $S$ we let $S^{\prime}:=B-S$ be the complement of $S$ in $B$. Writing $S^{\prime}=\left\{j_{1}, j_{2}, \ldots, j_{g+1}\right\}$ with $j_{1}<j_{2}<\ldots<j_{g+1}$, we define an element $\sigma_{S}$ in the symmetric group $S_{2 g+2}$ by:

$$
\sigma_{S}(k):=i_{k}, \quad \sigma_{S}(g+1+k):=j_{k} .
$$

3.3.5. Lemma. The two non-trivial so(2g+2)-invariant subspaces in $\wedge^{g+1} \mathbf{C}^{2 g+2}$ are:

$$
\left\langle\ldots, e_{S}+\operatorname{sgn}\left(\sigma_{S}\right) i^{g+1} e_{S}^{\prime}, \ldots\right\rangle_{S \ni 1} \quad \text { and } \quad\left\langle\ldots, e_{S}-\operatorname{sgn}\left(\sigma_{S}\right) i^{g+1} e_{S}^{\prime}, \ldots\right\rangle_{S \ni 1}
$$

where $S$ runs over the subsets of $B$ with $g+1$ elements with $1 \in S$.

Proof. (Cf. [FH], §19.2, Remarks(iii).) The quadratic form $Q$ defines an soequivariant isomorphism

$B: \wedge^{g+1} \mathbf{C}^{2 g+2} \longrightarrow\left(\wedge^{g+1} \mathbf{C}^{2 g+2}\right)^{*}=\wedge^{g+1}\left(\mathbf{C}^{2 g+2}\right)^{*}, \quad e_{S} \longmapsto \epsilon_{S}:=\epsilon_{i_{1}} \wedge \ldots \wedge \epsilon_{i_{g+1}}$

with $\left\{\ldots, \epsilon_{j}, \ldots\right\}$ the basis dual to the $e_{i}$. There is also a canonical (in particular so-equivariant) isomorphism:

$$
C: \wedge^{g+1} \mathbf{C}^{2 g+2} \longrightarrow\left(\wedge^{g+1} \mathbf{C}^{2 g+2}\right)^{*}, \quad \alpha \longmapsto\left[\beta \mapsto c_{\alpha, \beta}\right]
$$

with $c_{\alpha, \beta} \in \mathbf{C}$ defined by

$$
\alpha \wedge \beta=c_{\alpha, \beta} e_{1} \wedge e_{2} \wedge \ldots \wedge e_{2 g+2} .
$$

Thus we have an isomorphism $A:=B^{-1} \circ C: \wedge^{g+1} \mathbf{C}^{2 g+2} \rightarrow \wedge^{g+1} \mathbf{C}^{2 g+2}$ whose eigenspaces are so-invariant. An easy computation shows that the eigenvalues of $A$ are $\pm i^{g+1}$ and the eigenvectors are $e_{S} \pm \operatorname{sgn}\left(\sigma_{S}\right) i^{g+1} e_{S}^{\prime}$. 
3.3.6. A quartic polynomial. We consider the $g$-dimensional subspaces of $\mathbf{P}^{2 g+1}$ which are tangent to $Q_{z}$ :

$$
\bar{B}_{z}:=\left\{\mathbf{P}^{g} \subset \mathbf{P}^{2 g+1}: \operatorname{rank}\left(Q_{z} \text { restricted to } \mathbf{P}^{g}\right) \leq g\right\} \quad\left(\subset G r_{S L}\right) .
$$

The subvariety $\bar{B}_{z}$ is defined by a quadratic polynomial $P_{z}$ in the Plücker coordinates, that is, by a section in $H^{0}\left(G r_{S L}, \mathcal{O}_{G r_{S L}}(2)\right)$ ([vG], Thm 3):

$$
\bar{B}_{z}=Z\left(P_{z}\right), \quad P_{z} \in H^{0}\left(G r_{S L}, \mathcal{O}_{G r_{S L}}(2)\right) \subset S^{2}\left(\wedge^{g+1} \mathbf{C}^{2 g+2}\right) .
$$

This section is in fact the unique $S O_{z}$-invariant in $H^{0}\left(G r_{S L}, \mathcal{O}_{G r_{S L}}(2)\right)$, in case $g=2$ the quartic surface in $\mathbf{P} V$ defined by $P_{z}$ is the Kummer surface of the curve $\mathcal{C}_{z}([\mathrm{DR}])$.

3.3.7. Lemma. The variety $\bar{B}_{z}$ is defined by

$$
P_{z}=\sum_{S} z_{S} e_{S}^{2}
$$

where $S$ runs over the subsets with $g+1$ elements of $B=\{1, \ldots, 2 g+2\}$ and

$$
z_{S}:=z_{i_{1}} z_{i_{2}} \ldots z_{i_{g+1}}, \quad e_{S}=e_{i_{1}} \wedge \ldots \wedge e_{i_{g+1}} \quad \text { if } S=\left\{i_{1}, \ldots, i_{g+1}\right\} .
$$

Proof. From $[\mathrm{vG}]$, Theorem $3^{\prime}$ and its proof, we know that the trivial representation of $s o_{z}$ has multiplicity one in $H^{0}\left(G r_{S L}, \mathcal{O}_{G r_{S L}}(2)\right)=V\left(2 \lambda_{g+1}\right)$. This $s l_{2 g+2}$ representation corresponds to the partition $\mu: 2 g+2=2+2+\ldots+2$ and is thus realized as $\left(\mathbf{C}^{2 g+2}\right)^{\otimes 2 g+2} c_{\mu}$ where $c_{\mu}$ is the Young symmetrizer; one has in fact $V\left(2 \lambda_{g+1}\right) \subset S^{2}\left(\wedge^{g+1} \mathbf{C}^{2 g+2}\right)$ (see $[\mathrm{FH}], \S 15.5$, pp. 233-236). We use the standard tableau as in $[\mathrm{vG}]$ (and $[\mathrm{FH}]$ ) to construct $c_{\mu}$.

The trivial $\mathrm{so}_{z}$ sub-representation is obtained as follows. The quadratic form $Q_{z}$ defines the $s o_{z}$-invariant tensor $\sum_{i=1}^{2 g+2} z_{i} e_{i} \otimes e_{i} \in \mathbf{C}^{2 g+2} \otimes \mathbf{C}^{2 g+2}$, and we consider the $g+1$-fold tensor product of this tensor, where we insert the $k$-th factor in the positions $k$ and $g+1+k$ :

$\tau_{z}:=\sum_{i_{1}, \ldots, i_{g+1}=1}^{2 g+2} z_{i_{1}} z_{i_{2}} \ldots z_{i_{g+1}} e_{i_{1}} \otimes e_{i_{2}} \otimes \ldots \otimes e_{i_{g+1}} \otimes e_{i_{1}} \otimes \ldots \otimes e_{i_{g+1}}\left(\in\left(\mathbf{C}^{2 g+2}\right)^{\otimes 2 g+2}\right)$.

Thus $\tau_{z}$ is $s o_{z}$-invariant and it has the advantage that $\tau_{z} c_{\mu}$ is easily determined:

$$
\tau_{z} c_{\mu}=(c s t) \sum_{S} z_{S} e_{S} \cdot e_{S},
$$

with (cst) a non-zero integer and where we sum over all subsets $S$ with $g+1$ elements of $\{1, \ldots, 2 g+2\}$. (In fact, $c_{\mu}=a_{\mu} b_{\mu}$ and $a_{\mu}$ symmetrizes the indices $1, g+2 ; 2, g+3 ; \ldots ; g+1,2 g+2$ of a tensor (but $\tau_{z}$ is already symmetric in these indices) and next $b_{\mu}$ antisymmetrizes the first $g+1$ and last $g+1$ indices, giving the result above.)

\subsection{Verifying the contraction criterium.}

3.4.1. We need to determine the images of the $\Omega_{i j}$ 's in the representation $S^{2}\left(\wedge^{g+1} \mathbf{C}^{2 g+2}\right)$. In the standard representation $\mathbf{C}^{2 g+2}$ of $s o(2 g+2)$, with basis $e_{i}$ and dual basis $\epsilon_{i}$, we have:

$$
F_{i j}=2\left(e_{i} \otimes \epsilon_{j}-e_{j} \otimes \epsilon_{i}\right) \quad \in\left(\mathbf{C}^{2 g+2}\right) \otimes\left(\mathbf{C}^{2 g+2}\right)^{*}=\operatorname{End}\left(\mathbf{C}^{2 g+2}\right) .
$$

On $\wedge^{g+1} \mathbf{C}^{2 g+2}$, with basis $e_{S}=e_{i_{1}} \wedge \ldots \wedge e_{i_{g+1}}$ as before, $F_{i j}$ acts as

$F_{i j}\left(e_{i_{1}} \wedge \ldots \wedge e_{i_{g+1}}\right)=F_{i j}\left(e_{i_{1}}\right) \wedge e_{i_{2}} \wedge \ldots \wedge e_{i_{g+1}}+\ldots+e_{i_{1}} \wedge \ldots \wedge e_{i_{g}} \wedge F_{i_{j}}\left(e_{i_{g+1}}\right)$. 
Define a subset of $B=\{1, \ldots, 2 g+2\}$ by

$$
S+i j=(S \cup\{i, j\})-(S \cap\{i, j\}) .
$$

Then $F_{i j}\left(e_{S}\right)$ is, up to sign, $2 e_{S+i j}$ if $|S \cap\{i, j\}|=1$ and is zero otherwise:

$$
F_{i j}=2 \sum_{S,|S \cap\{i, j\}|=1} t_{S, i j} e_{S+i j} \otimes \epsilon_{S}
$$

with $t_{S, i j}= \pm 1$. Note that if $|S \cap\{i, j\}|=1$, then $F_{i j}^{2} e_{S}=-4 e_{S}$ so $t_{S, i j}=-t_{S+i j, i j}$.

3.4.2. Lemma. Let $W$ be a complex vector space, let $D \in W \otimes W^{*}$ be a linear differential operator and let $P \in S^{k} W$. Then the contraction of $P$ with the symbol of $D^{2}$ is given by:

$$
\sigma\left(D^{2}\right) \cdot P=2 D(P) D \quad \in S^{k-1} W \otimes W^{*} .
$$

Proof. The proof is straightforward using the conventions of 2.2.4 and 3.2.5.

3.4.3. Theorem. The composition, still denoted by $P_{z}$ :

$$
\mathcal{P} \longrightarrow S^{2} \wedge^{g+1} \mathbf{C}^{2 g+2} \longrightarrow S^{4} V\left(\omega_{g+1}\right), \quad z \longmapsto P_{z},
$$

with $P_{z}=\sum_{S} z_{S} e_{S}^{2}$ (as in Subsection 3.3.6), satisfies the differential equations:

$$
\left(\frac{1}{16} \sum_{j \neq i} \frac{\sigma \tilde{\rho}\left(\Omega_{i j}\right)}{z_{i}-z_{j}}\right) \cdot P_{z}+\left(\partial_{z_{i}} P_{z}\right) E=0 \quad(1 \leq i \leq 2 g+2)
$$

with $E=\sum e_{S} \otimes \epsilon_{S}$ the Euler vector field and

$$
\tilde{\rho}: U(s o(2 g+2)) \rightarrow \operatorname{End}\left(S^{2}\left(\wedge^{g+1} \mathbf{C}^{2 g+2}\right)\right) .
$$

Thus $P_{z}$ is a horizontal section for the connection $\nabla_{\lambda}^{W}$ with $W=S^{4} V\left(\omega_{g+1}\right)$ and $\lambda=-16$.

Proof. For symmetry reasons it suffices to verify the equation for $i=1$. By Lemma 3.4 .2

$$
\sigma \tilde{\rho}\left(\Omega_{1 j}\right) \cdot e_{S}^{2}=2 \rho\left(F_{1 j}\right)\left(e_{S}^{2}\right) \rho\left(F_{1 j}\right)=4 e_{S} \rho\left(F_{1 j}\right)\left(e_{S}\right) \rho\left(F_{1 j}\right) .
$$

To simplify notation we write $\Omega_{i j}$ for $\sigma \tilde{\rho}\left(\Omega_{i j}\right)$ and $F_{i j}$ for $\rho\left(F_{i j}\right)$ (as in 3.4.1). Then $F_{1 j}\left(e_{S}\right)=0$ unless $|S \cap\{1, j\}|=1$. Assume $1 \in S$ and $j \notin S$ (so $j \in B-S$ ) and consider the contraction of $\Omega_{1 j}$ with the term $z_{S} e_{S}^{2}+z_{S+i j} e_{S+i j}^{2}$ from $P_{z}$ :

$$
\begin{aligned}
\Omega_{1 j} \cdot\left(z_{S} e_{S}^{2}+z_{S+1 j} e_{S+1 j}^{2}\right) & =8\left(t_{S, 1 j} z_{S}+t_{S+1 j, 1 j} z_{S+1 j}\right) e_{S} e_{S+1 j} F_{1 j} \\
& =8 t_{S, 1 j}\left(z_{1} z_{\bar{S}}-z_{j} z_{\bar{S}}\right) e_{S} e_{S+1 j} F_{1 j} \\
& =\left(z_{1}-z_{j}\right) \Omega_{1 j} \cdot z_{\bar{S}} e_{S}^{2}
\end{aligned}
$$

where $\bar{S}:=S-\{1\}$. Therefore we get:

$$
\frac{\Omega_{1 j}}{z_{1}-z_{j}} \cdot P_{z}=\Omega_{1 j} \cdot\left(\sum_{S \ni 1, j \notin S} z_{\bar{S}} e_{S}^{2}\right) .
$$


Summing this result over all $j$ and changing the order of summation gives:

$$
\begin{aligned}
\left(\sum_{j \neq 1} \frac{\Omega_{1 j}}{z_{1}-z_{j}}\right) \cdot P_{z} & =\sum_{S \ni 1} z_{\bar{S}}\left(\sum_{j \in B-S} \Omega_{1 j} \cdot e_{S}^{2}\right) \\
& =8 \sum_{S \ni 1} z_{\bar{S}} e_{S}\left(\sum_{j \in B-S} t_{S, 1 j} e_{S+1 j} F_{1 j}\right)
\end{aligned}
$$

where, as before, $B=\{1, \ldots, 2 g+2\}$. On the other hand, since $P_{z}=\sum z_{S} e_{S}^{2}$, we have:

$$
\partial_{z_{1}} P_{z}=\sum_{S \ni 1} z_{\bar{S}} e_{S}^{2}
$$

Thus the theorem follows if we prove, for all $S$ with $1 \in S$ :

$$
2 e_{S} E+\sum_{j \in B-S} t_{S, 1 j} e_{S+1 j} F_{1 j}=0
$$

With the definition of $F_{1 j}$ we find:

$$
\begin{aligned}
\sum_{j \in B-S} t_{S, 1 j} e_{S+1 j} F_{1 j} & =\sum_{j \in B-S} t_{S, 1 j} e_{S+1 j}\left(\sum_{T,|T \cap\{1, j\}|=1} 2 t_{T, 1 j} e_{T+1 j} \otimes \epsilon_{T}\right) \\
& =2 \sum_{T}\left(\sum_{j \in B-S,|T \cap\{1, j\}|=1} t_{S, 1 j} t_{T, 1 j} e_{S+1 j} e_{T+1 j}\right) \otimes \epsilon_{T} .
\end{aligned}
$$

Note that $e_{S} E=\sum_{T} e_{S} e_{T} \otimes \epsilon_{T}$ (with $T \in B,|T|=g+1$ ). Comparing coefficients of $\epsilon_{T}$, it remains to prove that (for all $S \ni 1$ and all $T$ ):

$$
e_{S} e_{T}+\sum_{j \in B-S,|T \cap\{1, j\}|=1} t_{S, 1 j} t_{T, 1 j} e_{S+1 j} e_{T+1 j}=0 .
$$

We show that the relations for $1 \in T$ follow from those with $1 \notin T$. In fact, we only want this relation in $V\left(4 \omega_{g+1}\right)$, so we restrict ourselves to the subspace $V\left(2 \omega_{g+1}\right) \subset \wedge^{g+1} \mathbf{C}^{2 g+2}$. The kernel of the restriction map is $\left\langle\ldots, e_{T}-\right.$ $\left.\operatorname{sgn}\left(e_{T}\right) i^{g+1} e_{T^{\prime}}, \ldots\right\rangle$ (or the other space in Lemma 3.3.5; the argument we give leads to the same conclusion in both cases).

Consider a $T$ with $1 \in T$ and $j \notin T$. Then $1 \notin T+1 j$ and after restriction:

$$
e_{T}=\operatorname{sgn}\left(\sigma_{T}\right) i^{g+1} e_{T^{\prime}}, \quad e_{T+1 j}=\operatorname{sgn}\left(\sigma_{(T+1 j)^{\prime}}\right) i^{-(g+1)} e_{(T+1 j)^{\prime}}
$$

where ' stands for the complement in $B$. Since $(T+1 j)^{\prime}=T^{\prime}+1 j$, substituting these relations and multiplying throughout by $i^{g+1}$ we get:

$$
(-1)^{g+1} \operatorname{sgn}\left(\sigma_{T}\right) e_{S} e_{T^{\prime}}+\sum_{j \in B-S,|T \cap\{1, j\}|=1} t_{S, 1 j} t_{T, 1 j} \operatorname{sgn}\left(\sigma_{T^{\prime}+1 j}\right) e_{S+1 j} e_{T^{\prime}+1 j} .
$$

Next we observe that

$$
\operatorname{sgn}\left(\sigma_{T^{\prime}+1 j}\right)=(-1)^{g+1} t_{T, 1 j} t_{T^{\prime}, 1 j} \operatorname{sgn}\left(\sigma_{T}\right),
$$

so it suffices to consider the relations with $1 \notin T$. Let $T:=\left\{1=i_{1}, \ldots, i_{g+1}\right\}, T^{\prime}:=$ $\left\{j_{1}, \ldots, j_{g+1}\right\}$ with $j=j_{k}$ and $i_{l}<j<j_{l+1}$. To get $\sigma_{T^{\prime}+1 j}$, first apply the permutation $(1 g+2) \ldots(g+12 g+2)$ to $B$; the sign of this permutation is $(-1)^{g+1}$. Then apply $\sigma_{T}$. Next apply the cyclic permutation $\left(j_{1} \ldots j_{g+1} 1\right)$ (with sign $\left.(-1)^{g+1}\right)$ and finally apply the inverse of $\left(\begin{array}{llllll}j_{k} & \ldots & j_{g+1} & i_{2} & \ldots & i_{l}\end{array}\right)$ (with sign $\left.(-1)^{g-k+l}\right)$. The resulting permutation is $\sigma_{T^{\prime}+1 j}$ and has $\operatorname{sign}(-1)^{g+k+l} \operatorname{sgn}\left(\sigma_{T}\right)$. 
On the other hand, since $1 \in T, j \notin T$ we get $F_{1 j}\left(e_{T}\right)=-2(-1)^{l-1} e_{T+1 j}$, so $t_{T, 1 j}=(-1)^{l}$ and $F_{1 j}\left(e_{T^{\prime}}\right)=+2(-1)^{k-1} e_{T^{\prime}+1 j}$, so $t_{T^{\prime}, 1 j}=(-1)^{k-1}$. This gives the formula for $\operatorname{sgn}\left(\sigma_{T^{\prime}+1 j}\right)$.

From now on we consider only $T$ 's with $1 \notin T$ and $S$ with $1 \in S$. We show that the desired relation:

$$
e_{S} e_{T}+\sum_{i \in(B-S) \cap T} t_{S, 1 i} t_{T, 1 i} e_{S+1 i} e_{T+1 i}
$$

is a Plücker relation. Thus it holds in $H^{0}\left(G r_{S L}, \mathcal{O}(2)\right)$ and therefore also upon restriction to $G r_{S O} \subset G r_{S L}$.

Let $S=\left\{1=i_{1}, \ldots, i_{g+1}\right\}$ and consider the Zariski open subset $U \subset G r_{S L}$ of $g+1$-dimensional subspaces $W \subset \mathbf{C}^{2 g+2}$ with Plücker coordinate $p_{S} \neq 0$. Any such $W$ has a (unique) basis $\left\{\ldots, w_{i}, \ldots\right\}$ with:

$$
\begin{aligned}
& W=\left\langle w_{1}, \ldots, w_{g+1}\right\rangle \quad \text { and } \quad\left(w_{k}\right)_{i_{l}}=\delta_{k l} \\
& \left(S=\left\{1=i_{1}, \ldots, i_{g+1}\right\}, i_{1}<\ldots<i_{g+1}\right)
\end{aligned}
$$

where $\delta_{k l}$ is Kronecker's delta. Let $M$ be the $(g+1) \times(2 g+2)$ matrix whose rows are the $w_{i}$. Let $M_{i}$ be the $i$-th column of $M_{W}$ (note $M_{i_{j}}=f_{j}$, the $j$-th standard basis vector of $\left.\mathbf{C}^{g+1}\right)$. Then the Plücker coordinate $p_{k_{1}, \ldots, k_{g+1}}$ is the determinant of the $(g+1) \times(g+1)$ submatrix of $M$ whose $j$-th column is $M_{k_{j}}$; we write:

$$
p_{k_{1}, \ldots, k_{g+1}}=\operatorname{det}\left(M_{k_{1}}, \ldots, M_{k_{g+1}}\right) \quad \text { with } \quad k_{1}<\ldots<k_{g+1} .
$$

Let $j \notin S$, with $i_{l}<j<i_{l+1}$. Then $p_{S+1 j}=(-1)^{l-1}\left(M_{j}\right)_{1}$ since

$$
\begin{aligned}
& \operatorname{det}\left(f_{i_{2}}, \ldots, f_{i_{l}}, M_{j}, f_{i_{l+1}}, \ldots, f_{g+1}\right) \\
& \quad=(-1)^{l-1} \operatorname{det}\left(M_{j}, f_{i_{2}}, \ldots, f_{i_{l}}, f_{i_{l+1}}, \ldots, f_{g+1}\right)=(-1)^{l-1}\left(M_{j}\right)_{1} .
\end{aligned}
$$

As earlier, since $1 \in S$ we have $t_{S, 1 j}=-(-1)^{l-1}$, hence $p_{S+1 j}=-t_{S, 1 j}\left(M_{j}\right)_{1}$.

Let $T=\left\{j_{1}, \ldots, j_{g+1}\right\}$, let $T \cap S^{\prime}=\left\{a_{1}, \ldots, a_{q}\right\}$ and let $j=j_{n}=a_{k}$. Then $p_{T}=\operatorname{det}\left(M_{j_{1}}, \ldots, M_{j_{g+1}}\right)$ is, up to sign, the determinant of a $q \times q$ submatrix $N$ of the $(g+1) \times q$ matrix with columns $M_{a_{1}}, \ldots, M_{a_{q}}$; we write $p_{T}=t \cdot \operatorname{det}(N)$ with $t= \pm 1$. Then

$$
\begin{aligned}
p_{T+1 j} & =\operatorname{det}\left(f_{1}, M_{j_{1}}, \ldots, \widehat{M_{j_{n}}}, \ldots, M_{j_{g+1}}\right) \\
& =(-1)^{j_{n}-1} \operatorname{det}\left(M_{j_{1}}, \ldots, M_{j_{n-1}}, f_{1}, M_{j_{n+1}}, \ldots, M_{j_{g+1}}\right) \\
& =(-1)^{j_{n}-1}(-1)^{k+1} t \cdot \operatorname{det}\left(N^{1 k}\right)
\end{aligned}
$$

where $N^{1 k}$ is the $(q-1) \times(q-1)$ submatrix of $N$ obtained by deleting the first row and $k$-th column. Since $1 \notin T$, we have $t_{T, 1 j}=(-1)^{j_{n}-1}$ and thus $p_{T+1 j}=$ $t(-1)^{k+1} \operatorname{det}\left(N^{1 k}\right)$.

Substituting these expressions for $p_{S+1 j}$ and $p_{T+1 j}$ we get:

$$
1 \cdot t \cdot \operatorname{det}(N)+\sum_{j \in\left\{a_{1}, \ldots, a_{q}\right\}} t(-1)^{k}\left(M_{j}\right)_{1} \operatorname{det}\left(N^{1 k}\right)
$$

which is zero in virtue of a well known formula for the determinant. 


\section{The Heisenberg Group AND the SPIN REPRESENTATION}

4.1. We recall the basic facts on the Heisenberg group and we discuss the projective representation of its automorphism group. In Subsection 4.3 we relate the Heisenberg group (in its irreducible $2^{g}$-dimensional representation) with the (half) spin representation of the orthogonal group. Combining this with previous results, we can finally write down Hitchin's connection in Subsection 4.4.

\subsection{The Heisenberg group.}

4.2.1. Definitions. We introduce a variant of the Heisenberg group (cf. Subsection 2.5.2). For any positive integer $g$ we define a (finite) Heisenberg group $G$ by:

$$
G=G_{g}:=\left\{(t, x): t \in \mathbf{C}, t^{4}=1, \quad x=\left(\xi, \xi^{\prime}\right) \in \mathbf{F}_{2}^{g} \times \mathbf{F}_{2}^{g}\right\}
$$

with identity element $(1,0)$ and multiplication law:

$$
\left(t,\left(\xi, \xi^{\prime}\right)\right)\left(s,\left(\eta, \eta^{\prime}\right)\right):=\left(t s(-1)^{\xi \eta^{\prime}}, \xi+\eta, \xi^{\prime}+\eta^{\prime}\right), \quad(t, x)^{-1}=\left((-1)^{\xi \xi^{\prime}} t^{-1}, x\right)
$$

with $\xi \eta^{\prime}:=\sum_{i=1}^{g} \xi_{i} \eta_{i}^{\prime}$ and $x=\left(\xi, \xi^{\prime}\right)$. The group $G$ has $2^{2 g+2}$ elements, it is non-abelian, in fact its center is $\{(t, 0)\}$. We define a symplectic form on $\mathbf{F}_{2}^{2 g}$ by:

$$
E(x, y)=\xi \eta^{\prime}+\eta \xi^{\prime} ; \quad \text { then }(t, x)(s, y)(t, x)^{-1}(s, y)^{-1}=\left((-1)^{E(x, y)}, 0\right)
$$

where $x=\left(\xi, \xi^{\prime}\right), y=\left(\eta, \eta^{\prime}\right)$.

4.2.2. Representations. Let $V$ be the $2^{g}$-dimensional vector space of complex valued functions $\mathbf{F}_{2}^{g} \rightarrow \mathbf{C}$. It has a standard basis consisting of $\delta$-functions

$$
X_{\sigma}: \mathbf{F}_{2}^{g} \longrightarrow \mathbf{C}, \quad X_{\sigma}(\sigma)=1, \quad X_{\sigma}(\rho)=0 \quad \text { if } \sigma \neq \rho .
$$

The Heisenberg group $G$ has a representation $U$ on $V$, the Schrödinger representation:

$$
\left(U\left(t,\left(\xi, \xi^{\prime}\right)\right) f\right)(\sigma):=t(-1)^{\sigma \xi^{\prime}} f(\sigma+\xi)
$$

thus

$$
U\left(t,\left(\xi, \xi^{\prime}\right)\right) X_{\sigma}=t(-1)^{(\sigma+\xi) \xi^{\prime}} X_{\sigma+\xi} .
$$

For every $x \in \mathbf{F}_{2}^{2 g}-\{0\}$ choose a $\left(t_{x}, x\right) \in G$ of order two. We define:

$$
U_{x}:=U\left(t_{x}, x\right) \quad(\in G L(V)), \quad \text { so } \quad U_{x}^{2}=I
$$

and define $U_{0}=I$. Then $\operatorname{Im}(U)=\left\{t U_{x}: t^{4}=1, x \in \mathbf{F}_{2}^{2 g}\right\}$.

4.2.3. Automorphisms. We define a subgroup of $A u t(G)$ by:

$$
A(G):=\{\phi \in \operatorname{Aut}(G): \phi((t, 0))=(t, 0) \quad \forall t\} .
$$

For $\phi \in A(G)$ and $(t, x) \in G$ we can then write:

$$
\phi(t, x):=\left(f_{\phi}(x) t, M_{\phi}(x)\right), \quad \text { with } \quad M_{\phi}: \mathbf{F}_{2}^{2 g} \longrightarrow \mathbf{F}_{2}^{2 g}, \quad f_{\phi}: \mathbf{F}_{2}^{2 g} \longrightarrow \mathbf{C}^{*}
$$

$($ note $\phi(t, x)=\phi(t, 0) \phi(1, x)=(t, 0) \phi(1, x))$. The map $M: A(G) \rightarrow \operatorname{Aut}\left(\mathbf{F}_{2}^{2 g}\right), \phi \mapsto$ $M_{\phi}$ is a homomorphism, its image is $\operatorname{Sp}\left(\mathbf{F}_{2}^{2 g}, E\right)$ and $\operatorname{ker}(M) \cong G / \operatorname{Center}(G) \cong \mathbf{F}_{2}^{2 g}$ consists of interior automorphisms:

$$
0 \longrightarrow \mathbf{F}_{2}^{2 g} \longrightarrow A(G) \stackrel{M}{\longrightarrow} S p\left(2 g, \mathbf{F}_{2}\right) \longrightarrow 0 .
$$


4.2.4. A projective representation. The Schrödinger representation $U$ of $G$ on $V$ is the unique irreducible representation of $G$ in which $(t, 0)$ acts by multiplication by $t$. Given $\phi \in A(G)$, the representation $U \circ \phi$ enjoys the same property. Hence by Schur's lemma we get a linear map, unique up to scalar multiple,

$$
\tilde{T}_{\phi}: V \longrightarrow V, \quad \text { with } \quad \tilde{T}_{\phi} U(h)=U(\phi(h)) \tilde{T}_{\phi},
$$

for all $h \in G$. Note $\tilde{T}_{\phi}$ normalizes $\operatorname{Im}(U)$. In this way we get a projective representation of $A(G)$ :

$$
\tilde{T}: A(G) \longrightarrow P G L(V), \quad \phi \longmapsto \tilde{T}_{\phi} .
$$

When $\phi=\operatorname{Int}_{(t, y)}$ we may take $\tilde{T}_{\phi}=U_{y}$.

On the other hand, since $\operatorname{Im}(U) \cong G$, any $T \in G L(V)$ which normalizes $\operatorname{Im}(U)$ defines an automorphism $\phi_{T}$ of $G$. Since the center of $G$ acts by scalar multiples of the identity, we have $\phi_{T} \in A(G)$. Thus we get an exact sequence

$$
0 \longrightarrow \mathbf{C}^{*} \longrightarrow \text { Normalizer }_{G L(V)}(\operatorname{Im}(U)) \longrightarrow A(G) \longrightarrow 0 \text {. }
$$

4.2.5. Example. For $x=\left(\xi, \xi^{\prime}\right) \in \mathbf{F}_{2}^{2 g}-\{0\}$, we define the transvection

$$
T_{x}: \mathbf{F}_{2}^{2 g} \longrightarrow \mathbf{F}_{2}^{2 g}, \quad y \longmapsto y+E(y, x) x .
$$

Then $T_{x} \in S p\left(2 g, \mathbf{F}_{2}\right)$ and the (finite) symplectic group $S p\left(2 g, \mathbf{F}_{2}\right)$ is generated by transvections. Note that the transvections are involutions: $T_{x}^{2}=1$.

Let $x=\left(0, \xi^{\prime}\right)$ with $\xi^{\prime}=(1,0, \ldots, 0) \in \mathbf{F}_{2}^{g}$. On the basis $X_{(0, \ldots, 0)}, \ldots, X_{(0, \tau)}, \ldots$, $X_{(1,0, \ldots, 0)}, \ldots, X_{(1, \tau)}, \ldots$ with $\tau \in \mathbf{F}_{2}^{g-1}$ we have:

$$
U_{x}=\left(\begin{array}{cc}
I & 0 \\
0 & -I
\end{array}\right) ; \quad \text { let } \quad \tilde{T}_{x}:=\left(\begin{array}{cc}
I & 0 \\
0 & i I
\end{array}\right) .
$$

Then it is easy to verify that $\tilde{T}_{x} U_{y} \tilde{T}_{x}^{-1}=t_{x, y} U_{T_{x}(y)}$ for all $y \in \mathbf{F}_{2}^{2 g}$ and some $t_{x, y} \in \mathbf{C}^{*}$. Thus $\tilde{T}_{x}$ lies in $A(G)$ and $M\left(\tilde{T}_{x}\right)=T_{x}$.

\subsection{The spin representation.}

4.3.1. The two half spin representations of $s o(2 g+2)$ are each realized on a $2^{g}$ dimensional vector space. We recall, using the Clifford algebra, how they can be constructed using the Heisenberg group. We will have to consider the Heisenberg group $G_{g+1}$ which acts on a $2^{g+1}$-dimensional vector space. Elements of order two in the Heisenberg group will define the spin representation of $s o(2 g+2)$. Restriction to suitable subspaces will give the half spin representations and their relation with $G_{g}$ (a subquotient of $G_{g+1}$ ). This relation between $s o(2 g+2)$ and the Heisenberg group is used in 4.4.3 to prove the Heisenberg invariance of the flat connections introduced in 3.2.4.

4.3.2. In dealing with hyperelliptic curves and the half spin representation of the orthogonal group, the following (classical) notation for points in $\mathbf{F}_{2}^{2 g}$ is convenient ([DO], VIII.3; [M]). Let

$$
B:=\{1,2, \ldots, 2 g+2\}
$$

then

$$
F_{B}:=\left\{f: B \rightarrow \mathbf{F}_{2}: \sum_{b \in B} f(b)=0\right\} /\left\{0,[b \mapsto 1]_{b \in B}\right\}
$$


is an $\mathbf{F}_{2}$-vector space of dimension $2 g$. For a subset $T \subset B$ with an even number of elements we denote by $x_{T} \in F_{B}$ (or simply $T$ ) the element defined by the function $f$ with $f(b)=1$ iff $b \in T$. Note that $x_{T}=x_{T^{\prime}}$ when $T^{\prime}$ is the complement of $T$ in $B$, moreover

$$
x_{T}+x_{S}=x_{R} \quad \text { with } \quad R=T+S:=(T \cup S)-(T \cap S) .
$$

4.3.3. We fix the following isomorphism of $\mathbf{F}_{2}$-vector spaces, and identify them in this way in the remainder of the paper ([DO], VIII.3, Lemma 2, but note we interchanged $\left.\left(e_{i}, 0\right) \leftrightarrow\left(0, e_{i}\right)\right)$ :

$$
F_{B} \stackrel{\cong}{\longrightarrow} \mathbf{F}_{2}^{g} \times \mathbf{F}_{2}^{g}, \quad x_{\{2 i-1,2 i\}}=\left(0, e_{i}\right), \quad x_{\{2 i, 2 i+1, \ldots, 2 g+1\}}=\left(e_{i}, 0\right) \quad(1 \leq i \leq g) .
$$

The symplectic form $E$ on $\mathbf{F}_{2}^{g} \times \mathbf{F}_{2}^{g}$ can now be easily computed on $F_{B}$ by:

$$
E\left(x_{T}, x_{S}\right)=|T \cap S| \bmod 2 .
$$

4.3.4. To accomodate both $G_{g}$ and $G_{g+1}$ we extend the construction of Subsection 4.3.2. The inclusion

$$
B:=\{1, \ldots, 2 g+2\} \hookrightarrow B^{\sharp}:=\{1, \ldots, 2 g+4\}
$$

and extension by zero of functions on $B$ to functions on $B^{\sharp}$ induces

$$
\tilde{F}_{B}:=\left\{f: B \rightarrow \mathbf{F}_{2}: \sum_{b \in B} f(b)=0\right\} \hookrightarrow \tilde{F}_{B^{\sharp}}:=\left\{f: B \rightarrow \mathbf{F}_{2}: \sum_{b^{\prime} \in B^{\sharp}} f\left(b^{\prime}\right)=0\right\} .
$$

The function $g^{\prime} \in \tilde{F}_{B^{\sharp}}$ with $g^{\prime}\left(b^{\prime}\right)=1\left(\right.$ all $\left.b^{\prime} \in B^{\sharp}\right)$ does not lie in $\tilde{F}_{B}$, thus

$$
\tilde{F}_{B} \hookrightarrow F_{B^{\sharp}}:=\tilde{F}_{B^{\sharp}} /\left\{0, g^{\prime}\right\} .
$$

The image in $F_{B^{\sharp}}$ of the function $g\left(\in \tilde{F}_{B}\right)$ with $g(b)=1($ all $b \in B)$ is the element

$$
p^{\prime}:=x_{\{1,2, \ldots, 2 g+2\}}=x_{\{2 g+3,2 g+4\}} \in F_{B^{\sharp}} .
$$

Using the definition of the symplectic form on $F_{B^{\sharp}}$ (Subsection 4.3.3), which we denote by $E^{\sharp}$, one finds that:

$\tilde{F}_{B}=\left(p^{\prime}\right)^{\perp}, \quad$ with $\quad p^{\perp}:=\left\{x \in F_{B^{\sharp}}: E^{\sharp}\left(x, p^{\prime}\right)=0\right\}$ and $F_{B}=\left(p^{\prime}\right)^{\perp} /\left\{0, p^{\prime}\right\}$.

From Subsection 4.3.3 we have an identification:

$$
F_{B^{\sharp}}=\mathbf{F}^{g+1} \times \mathbf{F}^{g+1}, \quad p^{\prime}=(0,(1,1, \ldots, 1)) \quad\left(\in F_{B^{\sharp}}\right) .
$$

The Heisenberg group defined by this identification (cf. Subsection 4.2.1) will be denoted by $G^{\sharp}$, its Schrödinger representation by $U^{\sharp}$ (on the $2^{g+1}$-dimensional vector space $V^{\sharp}:=\left\{f: \mathbf{F}_{2}^{g+1} \rightarrow \mathbf{C}\right\}$ with basis of $\delta$-functions $\left.Y_{\sigma^{\prime}}, \sigma^{\prime} \in \mathbf{F}_{2}^{g+1}\right)$.

For any $x^{\prime} \in\left(p^{\prime}\right)^{\perp}\left(\subset F_{B^{\sharp}}\right)$, the maps $U_{p^{\prime}}^{\sharp}$ and $U_{x^{\prime}}^{\sharp}$ commute. Therefore the $U_{x^{\prime}}^{\sharp}$ with $x^{\prime} \in\left(p^{\prime}\right)^{\perp}$ act on the two eigenspaces of $U_{p^{\prime}}^{\sharp}$ which are:

$$
V_{+}^{\sharp}:=\left\langle\ldots, Y_{\sigma^{\prime}}, \ldots\right\rangle_{\sigma_{1}^{\prime}+\ldots+\sigma_{g+1}^{\prime}=0}, \quad V_{-}^{\sharp}:=\left\langle\ldots, Y_{\sigma^{\prime}}, \ldots\right\rangle_{\sigma_{1}^{\prime}+\ldots+\sigma_{g+1}^{\prime}=1} .
$$

A quotient map from $\left(p^{\prime}\right)^{\perp}\left(\subset\left(\mathbf{F}_{2}^{g} \times \mathbf{F}_{2}\right) \times\left(\mathbf{F}_{2}^{g} \times \mathbf{F}_{2}\right)\right)$ to $F_{B}=\mathbf{F}_{2}^{g} \times \mathbf{F}_{2}^{g}$ with kernel $\left\{0, p^{\prime}\right\}$ is:

$$
\left(p^{\prime}\right)^{\perp} \longrightarrow F_{B}=\left(p^{\prime}\right)^{\perp} /\left\{0, p^{\prime}\right\} \cong \mathbf{F}_{2}^{2 g}, \quad x^{\prime}:=\left(\left(a, a_{g+1}\right),\left(b, b_{g+1}\right)\right) \longmapsto x:=(a, \bar{b})
$$

with $\bar{b}=\left(b_{1}+b_{g+1}, \ldots, b_{g}+b_{g+1}\right)$. We define an isomorphism of vector spaces:

$$
V_{+}^{\sharp} \longrightarrow V, \quad Y_{\sigma_{1}, \ldots, \sigma_{g}, \sigma_{g+1}} \longmapsto X_{\sigma_{1}, \ldots, \sigma_{g}} .
$$


4.3.5. Lemma. For any $x^{\prime} \in\left(p^{\prime}\right)^{\perp}$ mapping to $x \in F_{B}$ there is a commutative diagram:

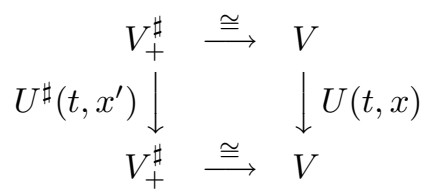

Moreover, if $1 \leq j, k \leq 2 g+1$, then restriction of $U_{x_{j k}}^{\sharp}$ to $V_{+}^{\sharp} \cong V$ is given by $\pm U_{x_{i j}}$.

Proof. The proof consists of easy computations which we leave to the reader.

4.3.6. The Clifford algebra. The Clifford algebra $C(Q)$ of the quadratic form $Q=$ $x_{1}^{2}+\ldots+x_{2 g+2}^{2}$ on $V_{Q}=\mathbf{C}^{2 g+2}$ is the quotient of the tensor algebra $T\left(V_{Q}\right)$ by the two-sided ideal $I$ generated by the elements $v \otimes v-Q(v)$ for $v \in V_{Q}([\mathrm{FH}], \S 20.1)$ :

$$
C(Q):=T\left(V_{Q}\right) / I=\left(\mathbf{C} \oplus V_{Q} \oplus V_{Q} \otimes V_{Q} \oplus \ldots\right) /(\ldots,-Q(v)+v \otimes v, \ldots)_{v \in V_{Q}} .
$$

Let $e_{1}, \ldots, e_{2 g+2}$ be the standard basis vectors of $V_{Q}$. Then the $C(Q)$ is generated by $\mathbf{C}$ and the $e_{j}$ with relations:

$$
e_{j} \cdot e_{j}=1, \quad e_{j} \cdot e_{k}+e_{k} \cdot e_{j}=0 \quad(j \neq k)
$$

here - stands for the product induced by $\otimes$ on $C(Q)$. The (associative) algebra $C(Q)$ becomes a Lie algebra by defining, as usual, the Lie bracket to be $[x, y]:=x \cdot y-y \cdot x$.

The following proposition relates the Heisenberg group, the Clifford algebra and the spin representation of the Lie algebra $s o(2 g+2)$.

4.3.7. Proposition. With the notation as above we have:

1. The $\mathbf{C}$-linear map

$$
\gamma^{\sharp}: C(Q) \longrightarrow \operatorname{End}\left(V^{\sharp}\right), \quad \lambda \mapsto \lambda I, \quad e_{k} \mapsto U_{x_{k, 2 g+4}}^{\sharp}
$$

$(\lambda \in \mathbf{C}, \quad k=1, \ldots, 2 g+2)$ defines an isomorphism of (associative $\mathbf{C}$-) algebras.

2. The linear map

$$
\rho_{s}^{\sharp}: s o(2 g+2) \longrightarrow \operatorname{End}\left(V^{\sharp}\right) \cong_{\gamma^{\sharp}} C(Q), \quad F_{j k} \longmapsto U_{x_{j, 2 g+4}}^{\sharp} U_{x_{k, 2 g+4}}^{\sharp}\left(=\gamma^{\sharp}\left(e_{j} \cdot e_{k}\right)\right)
$$

is an injective homomorphism of Lie algebras.

3. The subspace $V_{+}^{\sharp}$ of $V^{\sharp}$ is invariant under the action of so $(2 g+2)$. The Lie algebra representation

$$
\rho_{s}: s o(2 g+2) \longrightarrow \operatorname{End}\left(V_{+}^{\sharp}\right) \cong \operatorname{End}(V), \quad \rho_{s}(x):=\left.\rho_{s}^{\sharp}(x)\right|_{V_{+}^{\sharp}}
$$

is an (irreducible) half spin representation of so $(2 g+2)$. In particular, $V \cong$ $V\left(\omega_{g+1}\right)$.

4. We have

$$
\rho_{s}\left(F_{j k}\right)= \pm i U_{x_{j k}} \quad\left(F_{j k} \in s o(2 g+2), \quad i^{2}=-1\right),
$$

where the $U_{x_{j k}}(\in \operatorname{End}(V))$ with $x_{j k} \in \mathbf{F}_{2}^{2 g}$ are in the Schrödinger representation of the Heisenberg group $G$. 
Proof. (1) By definition we have $\left(U_{x^{\prime}}^{\sharp}\right)^{2}=I$ for all $x^{\prime} \in \mathbf{F}_{2}^{2 g+2}$. Moreover,

$$
E^{\sharp}\left(x_{\{k, 2 g+4\}}, x_{\{l, 2 g+4\}}\right)=1
$$

when $k \neq l$ so the corresponding maps anti-commute:

$$
U_{x_{k, 2 g+4}}^{\sharp} U_{x_{l, 2 g+4}}^{\sharp}=-U_{x_{l, 2 g+4}}^{\sharp} U_{x_{k, 2 g+4}}^{\sharp} .
$$

Then the map $\gamma^{\sharp}$ preserves the relations in $C(Q)$ and is thus an algebra homomorphism. It is surjective because the matrices $U_{x^{\prime}}^{\sharp}$, where $x^{\prime}$ runs over $\mathbf{F}_{2}^{g+1} \times \mathbf{F}_{2}^{g+1}$, are a basis of $\operatorname{End}\left(V^{\sharp}\right)$ (the Schrödinger representation being irreducible) and any $U_{x^{\prime}}^{\sharp}$ is a product (up to scalar multiple) of suitable $U_{x_{j, 2 g+4}}^{\sharp}$. Therefore $\gamma^{\sharp}$ is an isomorphism since both algebras have the same dimension.

(2) This is worked out in $[\mathrm{FH}]$, Lemma 20.7. With their notations and our $Q$, we have

$$
\begin{gathered}
\phi: \wedge^{2} V_{Q} \stackrel{\cong}{\cong} s o(2 g+2), \quad e_{j} \wedge e_{k} \longmapsto F_{j k}, \\
\psi: \wedge^{2} V_{Q} \longrightarrow C(Q), \quad e_{j} \wedge e_{k} \longmapsto e_{j} \cdot e_{k} \quad(j \neq k), \quad \text { and } \quad \rho_{s}^{\sharp}=\psi \circ \phi^{-1} .
\end{gathered}
$$

(3) By definition of $\rho_{s}^{\sharp}$ and the fact that $U^{\sharp}$ is a representation we have:

$$
\rho_{s}^{\sharp}\left(F_{j k}\right)=U_{x_{j, 2 g+4}}^{\sharp} U_{x_{k, 2 g+4}}^{\sharp}=c_{j k} U_{x_{j k}}^{\sharp},
$$

with $1 \leq j, k \leq 2 g+2$ and some $c_{j k} \in \mathbf{C}^{*}$. Thus the $\rho_{s}^{\sharp}\left(F_{j k}\right)$ commute with $U_{p^{\prime}}^{\sharp}$ in $\operatorname{End}\left(V^{\sharp}\right)$. Therefore we obtain a Lie algebra representation on $V_{+}^{\sharp}$; cf. [FH], Prop. 20.15 (and identify their $\operatorname{End}\left(\wedge^{\text {even }} W\right.$ ) with our $\left.\operatorname{End}\left(V_{+}^{\sharp}\right)\right)$, where also the irreducibility is proved.

(4) We have $\rho_{s}^{\sharp}\left(F_{j k}\right)=U_{x_{j, 2 g+4}}^{\sharp} U_{x_{k, 2 g+4}}^{\sharp}$, thus $\rho_{s}^{\sharp}\left(F_{j k}\right)^{2}=-I$ since these two elements anti-commute. Therefore $\rho_{s}^{\sharp}\left(F_{j k}\right)= \pm i U_{x_{j k}}^{\sharp}$, which acts as $\pm i U_{x_{j k}}$ on $V_{+}^{\sharp}$.

\subsection{The Hitchin connection.}

4.4.1. The symmetric group $S_{2 g+2}$ acts on $\mathcal{P}$ by permuting the coordinates; the quotient will be denoted by $\overline{\mathcal{P}}$. The fundamental group of $\mathcal{P}$ is the pure braid group and $\pi_{1}(\overline{\mathcal{P}})=B_{2 g+2}$, the braid group.

In case $g=2$, we have $S_{6} \cong S p\left(4, \mathbf{F}_{2}\right)$, in fact there is a surjective map (which factors over the mapping class group)

$$
\pi_{1}(\overline{\mathcal{P}})=B_{6} \longrightarrow S p(4, \mathbf{Z}) ;
$$

the kernel of the composition $B_{6} \rightarrow S p(4, \mathbf{Z}) \rightarrow S p\left(4, \mathbf{F}_{2}\right)$ is $\pi_{1}(\mathcal{P})$.

From the theory of theta functions we know that the group scheme $\mathcal{G}$ is trivialized on the cover $\tilde{\mathcal{P}}$ of $\mathcal{P}$ defined by the kernel of the composition:

$$
\phi: \pi_{1}(\overline{\mathcal{P}}) \longrightarrow S p(4, \mathbf{Z}) \longrightarrow S p(4, \mathbf{Z}) / \Gamma_{2}(2,4) \cong A(G) .
$$

Here we use Igusa's notation:

$$
\begin{aligned}
\Gamma_{2}(2,4):= & \left\{\left(\begin{array}{cc}
I+2 A & 2 B \\
2 C & I+2 D
\end{array}\right) \in S p(4, \mathbf{Z}):\right. \\
& \quad \operatorname{diagonal}(B) \equiv \operatorname{diagonal}(C) \equiv(0,0) \quad \bmod 2\} .
\end{aligned}
$$


Thus we consider the following diagram of étale Galois coverings:

$$
\widetilde{\mathcal{P}} \longrightarrow \mathcal{P} \longrightarrow \overline{\mathcal{P}}:=\mathcal{P} / S_{6},
$$

and the corresponding exact sequence of covering groups:

$$
0 \longrightarrow \mathbf{F}_{2}^{2 g} \longrightarrow A(G) \longrightarrow S_{6} \longrightarrow 0 .
$$

Recall that we have defined a projective representation $\tilde{T}: A(G) \rightarrow P G L(V)$ in 4.2.4. This induces projective representations on each $S^{k} V$.

4.4.2. Theorem. With the notation as in 4.4 .1 (except for $S_{k}$ ) and $g=2$ we have:

1. There is a line bundle $\mathcal{N}$ on $\widetilde{\mathcal{P}}$ such that the pullback of $p_{*} \mathcal{L}^{\otimes k}$ is isomorphic to $S_{k} \otimes_{\mathbf{C}} \mathcal{N}$. Here $S_{k}=S^{k} V\left(\omega_{g+1}\right)$ and $V\left(\omega_{g+1}\right)$ is a half spin representation of $s o(6) \cong s l(4)$ (which is the standard representation (or its dual) of $\operatorname{sl}(4)$ ).

2. The Hitchin connection on the pullback of $p_{*} \mathcal{L}^{\otimes k}$ to $\widetilde{\mathcal{P}}$ is given by the pullback to $\widetilde{\mathcal{P}}$ of the connection on $S_{k} \otimes_{\mathbf{C}} \mathcal{O}_{\mathbf{P}}$ defined by the one-form

$$
\frac{-1}{16(k+2)} \sum_{i, j i \neq j} \frac{\sigma \tilde{\rho_{s}}\left(\Omega_{i j}\right) \mathrm{d} z_{i}}{z_{i}-z_{j}}
$$

with $\tilde{\rho}_{s}: U(s o(2 g+2)) \rightarrow \operatorname{End}\left(S_{1}\right)$ the half spin representation (then the $\sigma \tilde{\rho_{s}}\left(\Omega_{i j}\right)$ give endomorphisms of each $S_{k} ;$ cf. 3.2.5).

3. The Hitchin connection on the bundle $S_{k} \otimes \mathcal{O}_{\widetilde{\mathcal{P}}}$ over $\widetilde{\mathcal{P}}$ descends to a projective flat connection on the the bundle $\left(S_{k} \otimes \mathcal{O}_{\widetilde{\mathcal{P}}}\right) / A(G)$ over $\overline{\mathcal{P}}$, where $A(G)$ acts on $S_{k}$ via the $k$-th symmetric power of its projective representation on $S_{1}$ and $A(G)$ acts on $\widetilde{\mathcal{P}}$ as $\operatorname{Gal}(\widetilde{\mathcal{P}} / \overline{\mathcal{P}})$. This descent gives the $k$-th Hitchin connection of the natural descent of the family of curves described in 1.4.

Proof. (1) For the first part it suffices to show that the pullback of $p: \mathbf{P} \rightarrow \mathcal{P}$ to $\tilde{\mathcal{P}}$ is trivial. Since $\mathcal{G}$ is isomorphic to the constant group scheme $G$ (cf. 4.2 .1 (with $t \in \mathbf{C}^{*}$ here)), the uniqueness of the Schrödinger representation gives the global trivialization. We already observed that the line bundle $\mathcal{N}$ does not interfere with the projective connections.

(2) We use Theorem 2.6.9 so we must verify (Eq.) from 2.6.7. The covariant derivatives given are defined by the heat operator

$$
X=-\frac{1}{16(k+2)} \sum_{j \neq i} \sigma \tilde{\rho}_{s}\left(\Omega_{i j}\right) \otimes \frac{\mathrm{d} z_{i}}{z_{i}-z_{j}} \quad\left(\in\left(S_{2} \otimes_{\mathbf{C}} S_{2}^{*}\right) \otimes \Omega_{\mathcal{P}}^{1}\right) .
$$

The Heisenberg-invariance of this operator follows from the facts that $\Omega_{i j}=F_{i j} \otimes$ $F_{i j}$, that $\rho_{s}\left(F_{k j}\right)= \pm i U_{x_{j k}}$ with $i^{2}=-1$ and that $U_{y} U_{x} U_{y}^{-1}= \pm U_{x}$. Since $S^{4}\left(\tilde{\rho}_{s}\right)$ is a subrepresentation of $\tilde{\rho}: s o(2 g+2) \rightarrow \operatorname{End} S^{2}\left(\wedge^{2} \mathbf{C}^{2 g+2}\right)$ we have $\sigma \tilde{\rho_{s}}\left(\Omega_{i j}\right)=$ $\sigma \tilde{\rho}\left(\Omega_{i j}\right)$ on $S_{4}$. For the connection $\nabla_{0}$ on $\tilde{p}_{*} \mathcal{O}_{\mathbf{P}}(1)=S_{1} \otimes \mathcal{O}_{\tilde{P}}$ we simply take $\nabla_{0}(f w)=w \otimes \mathrm{d} f$. Then the equation $(E q$.$) is exactly the statement of Theorem$ 3.4 .3 .

(3) We have a smooth projective family of genus 2 curves over the scheme $\overline{\mathcal{P}}$ given by

$$
y^{2}=f(x)=x^{6}+a_{1} x^{5}+a^{2} x^{4}+\ldots+a_{6}=\prod_{i}\left(x-z_{i}\right)
$$


where $a_{i}$ is the $i$-th symmetric function of the $z_{j}$. Thus by the general theory of Section 2 we have a $\mathbf{P}^{3}$-bundle $\bar{p}: \overline{\mathbf{P}} \longrightarrow \overline{\mathcal{P}}$ and a projective Hitchin connection $\overline{\mathbf{D}}_{k}$ on $\bar{p}_{*} \mathcal{O}(k)$. Let $\varphi: \tilde{\mathcal{P}} \longrightarrow \overline{\mathcal{P}}$ be the natural map. Then there is an isomorphism

$$
\varphi^{*}(\overline{\mathbf{P}})=\mathbf{P}^{3} \times \tilde{\mathcal{P}}
$$

which is compatible with the action of $A(G)$ (which lies over the action of $A(G)$ on $\tilde{\mathcal{P}})$ on both sides: this isomorphism is given by the trivialization of the action of the theta-group scheme $\mathcal{G}$ which was mentioned earlier. The isomorphism $(*)$ induces an isomorphism

$$
(* *) \quad \varphi^{*}\left(\bar{p}_{*} O(k)\right) \cong S_{k} \otimes O_{\tilde{\mathcal{P}}}
$$

which is compatible with the natural projective action of $A(G)$ on the LHS (via $\mathcal{G}$ ) and the action of $A(G)$ on the RHS via the $\mathrm{Sym}^{k}$ of its natural representation on $S_{1}$. The heat operator given by $X$ (as in the proof of 4.4.2.2, see 2.6.7) on the LHS of $(*)$ is VIA $(*)$ compatible with the pullback of the Hitchin heat operator of the LHS of $(*)$. But both heat operators are invariant under the action of $A(G)$. Thus the projective connections on the RHS and LHS of $(* *)$ agree and are compatible with the projective action of $A(G)$ on both sides. In other words the natural descent datum on the LHS of $(* *)$ agrees with the descent datum on the RHS of $(* *)$.

4.4.3. Examples. We give some examples of the $\rho_{s}\left(\Omega_{i j}\right)$ 's in case $g=2$. We identify $V=S_{1}$, with its standard basis $X_{\sigma}, \sigma \in \mathbf{F}_{2}^{2}$. A linear map with matrix $\left(a_{k l}\right)$ is then also given by the linear differential operator $\sum_{k l} a_{k l} X_{k} \partial_{l}$ (since $\partial_{l}\left(X_{m}\right)=0$ for $l \neq m$ and is 1 if $l=m$ ). Recall from 4.3.3 that (for $g=2$ ):

$$
x_{12}=((0,0),(1,0)), \quad \text { so } \quad U_{x_{12}}=X_{00} \partial_{00}+X_{01} \partial_{01}-X_{10} \partial_{10}-X_{11} \partial_{11} .
$$

Similarly, we had $x_{16}=((1,0),(0,0))$ and $x_{26}=((1,0),(1,0))$ and so:

$$
\begin{aligned}
& U_{x_{16}}=X_{10} \partial_{00}+X_{11} \partial_{10}+X_{00} \partial_{10}+X_{01} \partial_{10}, \\
& U_{x_{26}}=i\left(-X_{10} \partial_{00}-X_{11} \partial_{10}+X_{00} \partial_{10}+X_{01} \partial_{10}\right) .
\end{aligned}
$$

Since $U_{x_{j k}}= \pm i \rho_{s}\left(F_{j k}\right)$ and $U_{x_{j k}}^{2}=I$ we get (cf. 3.2.5):

$$
\begin{aligned}
& \sigma\left(\tilde{\rho}_{s}\left(\Omega_{12}\right)\right) \\
& \quad=-2\left(X_{00}^{2} \partial_{00}^{2}+X_{01}^{2} \partial_{01}^{2}+X_{10}^{2} \partial_{10}^{2}+X_{11}^{2} \partial_{11}^{2}+2 X_{00} X_{01} \partial_{00} \partial_{01}-2 X_{10} X_{11} \partial_{10} \partial_{11}\right) .
\end{aligned}
$$

\subsection{Local monodromy.}

4.5.1. Introduction. We want to obtain some information on the representation of the mapping class group for genus two curves defined by Hitchin's connection. This representation has been studied, for arbitrary $g$ and $k$, by Moore and Seiberg [MS], Kohno [K2] and in the case $k=2$ by Wright [Wr]. In some sense, we merely find (weaker, local) results which do agree with their results.

4.5.2. The method. We recall how to determine the local monodromy on the trivial bundle $\mathcal{O}_{\mathcal{P}} \otimes_{\mathbf{C}} W$ with a connection $\nabla$. We consider a holomorphic map

$$
\phi: D^{*} \longrightarrow \mathcal{P}, \quad \text { with } \quad D^{*}:=\{t \in \mathbf{C}-\{0\}:|t|<\epsilon\}
$$

(for small, positive $\epsilon$ ) and pullback the one-form $\omega \in \operatorname{End}(W) \otimes \Omega_{\mathcal{P}}^{1}$ which defines the connection (so $\nabla(f w)=w \mathrm{~d} f-f \omega(w))$. We write

$$
\phi^{*} \omega=\left(\frac{R}{t}+A(t)\right) \mathrm{d} t, \quad R \in \operatorname{End}(W)
$$


with $A(t)$ holomorphic for $t=0$, and $R$ is called the residue at $t=0$. We identify $\pi_{1}\left(D^{*}\right)=\mathbf{Z} ; 1 \in \mathbf{Z}$ represents a small circle traversed in anti-clockwise direction. The eigenvalues of the monodromy of $1 \in \pi_{1}\left(D^{*}\right)$ are the $\exp \left(-2 \pi i \mu_{j}\right)$ were the $\mu_{i}$ are the eigenvalues of $R$ on $W$.

In case the monodromy transformations are semi-simple, the local monodromy is conjugated to $\exp (-2 \pi i R)$ (cf. [D], p. 54). However we could not prove the semi-simplicity (but it seems to be known to the physicists).

To get the eigenvalues of the monodromy of the Hitchin connection for a $\gamma \in$ $\pi_{1}(\overline{\mathcal{P}})$, let $n$ be the order of its image in $\operatorname{Gal}(\mathcal{P} / \overline{\mathcal{P}})$ and let $\bar{\gamma}$ be its image in $A(G)=$ $\operatorname{Gal}(\widetilde{\mathcal{P}} / \overline{\mathcal{P}})$. We choose a $\phi: D^{*} \rightarrow \mathcal{P}$ in such a way that $\phi_{*}(1)=\gamma^{n}\left(\in \pi_{1}(\mathcal{P})\right)$. Then these eigenvalues are the eigenvalues of the matrix $\exp \left(\frac{-2 \pi i}{n} R\right) \tilde{T}_{\bar{\gamma}}$, with $R$ determined as above for the corresponding connection on $\mathcal{P}$ (cf. [K1]). Since $\tilde{T}$ is a projective representation (on $S_{1}$ and thus on any $S_{k}$ ), the set of eigenvalues are only defined up to multiplication by one non-zero constant. This corresponds to the fact that we only have a projectively flat connection.

4.5.3. Non-separating vanishing cycle. Let $\gamma \in \pi_{1}(\overline{\mathcal{P}})$ such that $\gamma^{2}=\phi_{*}(1)$ with

$$
\phi: D^{*} \longrightarrow \mathcal{P}, \quad t \longmapsto\left(t+z_{2}, z_{2}, \ldots, z_{6}\right)
$$

where we fix distinct $z_{j} \in \mathbf{C}$. (Then $\gamma$ corresponds to a Dehn twist in a simple nonseparating loop in the mapping class group.) The residue of $\phi^{*} \omega$ for the connection on $S_{k} \otimes \mathcal{O}_{\mathcal{P}}$ corresponding to the Hitchin connection on $p_{*} \mathcal{L}^{\otimes k}$ is (cf. 4.4.2 and 4.4.3): $\frac{-1}{16(k+2)} \sigma \tilde{\rho_{s}}\left(\Omega_{12}\right)$,

$$
-\tilde{\rho}_{s}\left(\Omega_{12}\right)=R_{12}:=\left(\left(X_{00} \partial_{00}+X_{01} \partial_{01}\right)-\left(X_{10} \partial_{10}+X_{11} \partial_{11}\right)\right)^{2} .
$$

4.5.4. Lemma. The eigenvalues of the monodromy of $\gamma$ as in 4.5.3 on $S_{k}$ are:

$$
\lambda_{c}:=\exp \left(-2 \pi i \frac{c(c+1)}{k+2}\right), \quad \operatorname{mult}\left(\lambda_{c}\right)=(k-2 c+1)(2 c+1)
$$

(up to multiplication by a non-zero constant independent of $c$ ), with $2 c \in \mathbf{Z}$ and $0 \leq c \leq \frac{k}{2}$.

Proof. Since the eigenvalues are only determined up to a constant and since the difference between $2 R_{12}$ and $\sigma\left(R_{12}\right)$ is a multiple of the Euler vector field, which acts by multiplication by $k$ on $S_{k}$, it suffices to consider the eigenvalues of $R_{12}$. For a monomial in $S_{k}$ we have:

$$
R_{12}\left(X_{00}^{l_{0}} X_{01}^{l_{1}} X_{10}^{m_{0}} X_{11}^{m_{1}}\right)=\left(\left(l_{0}+l_{1}\right)-\left(m_{0}+m_{1}\right)\right)^{2}\left(X_{00}^{l_{0}} X_{01}^{l_{1}} X_{10}^{m_{0}} X_{11}^{m_{1}}\right) .
$$

Thus each monomial is an eigenvector. Let $b:=m_{0}+m_{1}$. Since the monomial has degree $k$, its eigenvalue is $(k-2 b)^{2}$. The multiplicity of the eigenvalue is $(k-b+1)(b+1)$ (the dimension of the space of homogeneous polynomials in 2 variables of degree $c$ is $c+1)$. Thus the eigenvalue $2(k-2 b)^{2}$ of $2 R_{12}$ has multiplicity $(k-b+1)(b+1)$.

The image $\bar{\gamma}$ of $\gamma$ in $A(G)$ is $\tilde{T}_{x_{12}}$ which acts on such a monomial by (cf. 4.2.5):

$$
\tilde{T}_{x_{12}}\left(X_{00}^{l_{0}} X_{01}^{l_{1}} X_{10}^{m_{0}} X_{11}^{m_{1}}\right)=i^{b}\left(X_{00}^{l_{0}} X_{01}^{l_{1}} X_{10}^{m_{0}} X_{11}^{m_{1}}\right) .
$$


The eigenvalues of $\gamma$ on $S_{k}$ are then, with $c:=b / 2$ :

$$
\begin{aligned}
\exp ( & \left.\frac{-2 \pi i}{2}\left(\frac{+1}{16(k+2)} 2(k-2 b)^{2}+\frac{b}{4}\right)\right) \\
& =\exp \left(-2 \pi i\left(\frac{k^{2}-8 c k+16 c^{2}+8 c k+16 c}{16(k+2)}\right)\right) \\
& =\exp \left(-2 \pi i\left(\frac{k^{2}}{16(k+2)}\right)\right) \exp \left(-2 \pi i\left(\frac{c(c+1)}{k+2}\right)\right) .
\end{aligned}
$$

4.5.5. Separating vanishing cycle. Now we consider a $\gamma \in \pi_{1}(\overline{\mathcal{P}})$ such that $\gamma=\phi_{*}(1)$ with

$$
\phi: D^{*} \longrightarrow \mathcal{P}, \quad t \longmapsto\left(t z_{1}, t z_{2}, t z_{3}, z_{4}, z_{5}, z_{6}\right),
$$

with distinct non-zero $z_{i}$ 's.

The residue in $t=0$ of the connection on $S_{k} \otimes \mathcal{P}$ corresponding to Hitchin's connection is

$$
\frac{-1}{16(k+2)} R_{123}, \quad \text { with } \quad R_{123}:=2 \sigma\left(\tilde{\rho_{s}}\left(\Omega_{12}+\Omega_{13}+\Omega_{23}\right)\right) .
$$

4.5.6. Lemma. Let $g=2$.

1. There are constants $\lambda_{k} \in \mathbf{Q}$ such that $\left(\right.$ in $\left.\operatorname{End}\left(S_{k}\right)\right)$ :

$$
R_{123}=16 R_{Q}+\lambda_{k} I \quad \text { with } \quad R_{Q}=\left(X_{00} X_{10}-X_{01} X_{11}\right)\left(\partial_{00} \partial_{10}-\partial_{01} \partial_{11}\right) .
$$

2. We define a subspace of $S_{k}$ by:

$$
V_{k}:=\operatorname{Kernel}\left(X_{Q}:=\partial_{00} \partial_{10}-\partial_{01} \partial_{11}: S_{k} \longrightarrow S_{k-2}\right) .
$$

Then the vector space $S_{k}$ is a direct sum:

$$
S_{k}=V_{k} \oplus Q V_{k-2} \oplus Q^{2} V_{k-4} \oplus \ldots .
$$

Moreover, each subspace $Q^{l} V_{k-2 l}$ is an eigenspace of $R_{Q}$ with eigenvalue:

$$
\lambda_{l}=l(k-l+1) \quad \text { and } \quad \operatorname{dim} V_{k-2 l}=(k-2 l+1)^{2} .
$$

3. The eigenvalues of of the monodromy of $\gamma$ on $S_{k}$ are

$$
\lambda_{l}:=\exp \left(-2 \pi i \frac{l(l+1)}{k+2}\right) \quad \operatorname{mult}\left(\lambda_{l}\right)=(k-2 l+1)^{2}
$$

(up to multiplication by a constant independent of $l$ ), with $l \in \mathbf{Z}$ and $0 \leq l \leq$ $k / 2$.

Proof. (1) Recall that $x_{12}$ corresponds to $((0,0),(1,0)) \in \mathbf{F}_{2}^{2} \times \mathbf{F}_{2}^{2}$ and $x_{23}=$ $x_{\{2,3,4,5\}}+x_{\{4,5\}}$ corresponds to $((1,1),(0,0))$, thus $x_{13}=x_{12}+x_{23}$ corresponds to $((1,1),(1,0))$. Since $(1,1)(1,0)=1$, we have a $+\operatorname{sign}$ in front of $\Omega_{13}$ below. Let $\tilde{\rho_{s}}\left(\Omega_{00}\right)$ be the square of the Euler vector field (which acts as $k^{2} I$ on $S_{k}$ ). Then:

$$
\begin{aligned}
& \tilde{\rho_{s}}\left(\Omega_{00}\right)=+\left(X_{00} \partial_{00}+X_{01} \partial_{01}+X_{10} \partial_{10}+X_{11} \partial_{11}\right)^{2}, \\
& \tilde{\rho}_{s}\left(\Omega_{12}\right)=-\left(X_{00} \partial_{00}+X_{01} \partial_{01}-X_{10} \partial_{10}-X_{11} \partial_{11}\right)^{2}, \\
& \tilde{\rho_{s}}\left(\Omega_{23}\right)=-\left(X_{11} \partial_{00}+X_{10} \partial_{01}+X_{01} \partial_{10}+X_{00} \partial_{11}\right)^{2}, \\
& \tilde{\rho}_{s}\left(\Omega_{13}\right)=+\left(X_{11} \partial_{00}+X_{10} \partial_{01}-X_{01} \partial_{10}-X_{00} \partial_{11}\right)^{2} .
\end{aligned}
$$

The sum of these operators, minus the (degree one) parts which act as constants on each $S_{k}$, is $4 R_{Q}$. Remembering the factor 2 in $R_{123}$ and in our symbols, we find the first statement. 
(2) This is well-known. Let $f \in V_{n}$, so $f$ is homogeneous of degree $n$ and $X_{Q}(f)=0$. Then an elementary computation gives for $l \geq 1$ (with variables $X_{i}$ and $\left.Q=X_{0} X_{1}-X_{2} X_{3}\right)$ :

$$
\begin{aligned}
\left(\partial_{0} \partial_{1}-\right. & \left.\partial_{2} \partial_{3}\right)\left(f Q^{l}\right) \\
= & \left(\partial_{0} f\right)\left(\partial_{1} Q^{l}\right)+\left(\partial_{0} Q^{l}\right)\left(\partial_{1} f\right)-\left(\partial_{2} f\right)\left(\partial_{3} Q^{l}\right) \\
& -\left(\partial_{2} Q^{l}\right)\left(\partial_{3} f\right)+f\left(\partial_{0} \partial_{1}-\partial_{2} \partial_{3}\right)\left(Q^{l}\right) \\
= & l Q^{l-1}\left(X_{0} \partial_{0}+X_{1} \partial_{1}+X_{2} \partial_{2}+X_{3} \partial_{3}\right)(f)+l(l+1) f Q^{l-1} \\
= & l(n+l+1) f Q^{l-1} .
\end{aligned}
$$

Writing $n=k-2 l$ we get, for all integers $l$ with $0 \leq 2 l \leq k$ :

$$
R_{Q}\left(f Q^{l}\right)=l(k-l+1) f Q^{l}, \quad f \in V_{k-2 l} .
$$

Hence each $V_{k-2 l} Q^{l}$ is an eigenspace for $R_{Q}$. By induction (the cases $k=0,1$ being trivial) we may assume that $S_{k-2}=\bigoplus S_{k-2-2 l} Q^{l}$. Therefore $Q S_{k-2}=$ $\bigoplus Q^{l+1} S_{k-2(l+1)} \subset S_{k}$ is a direct sum of eigenspaces of $R_{Q}$ and none of the eigenvalues of $R_{Q}$ on this subspace is zero $(k+l(l+1)>0$ for $k \geq 2, l \geq 0)$. Thus $\operatorname{ker}\left(R_{Q}\right) \cap Q S_{k-2}=\{0\}$ and $R_{Q}$ induces an isomorphism on $Q S_{k-2}$. Therefore $S_{k}=\operatorname{Ker}\left(R_{Q}\right) \oplus \operatorname{Im}\left(R_{Q}\right)=\bigoplus Q^{l} S_{k-2 l}$. The dimension of $V_{k}$ is then $\operatorname{dim} S_{k}-\operatorname{dim} S_{k-2}=\left(\begin{array}{c}k+3 \\ 3\end{array}\right)-\left(\begin{array}{c}k+1 \\ 3\end{array}\right)=(k+1)^{2}$.

(3) The image of $\gamma$ in $A(G)$ is trivial. The eigenvalues of $R_{123}=16 R_{Q}$ on $S_{k}$ are $16 l(k-l+1)$. Thus the eigenvalues of $\gamma$ are (see 4.5.2):

$$
\begin{aligned}
\exp \left(-2 \pi i \frac{-1}{16(k+2)} 16 l(k-l+1)\right) & =\exp \left(-2 \pi i\left(\frac{l^{2}-l-l(k+2)+2 l}{k+2}\right)\right) \\
& =\exp \left(-2 \pi i \frac{l(l+1)}{k+2}\right) .
\end{aligned}
$$

4.5.7. Kohno's results. The monodromy of the Hitchin connection has been studied by Moore-Seiberg and Kohno (among others); we will relate the results above to those contained in [K2] for $g=2$.

Let $\gamma$ be a tri-valent graph with two vertices, so there are three edges meeting in each vertex. For $k \in \mathbf{Z}_{\geq 0}$ we define a finite set $b_{k}(\gamma)$ of functions on the edges of $\gamma$ by:

$$
f: \operatorname{Edges}(\gamma) \longrightarrow\left\{0, \frac{1}{2}, 1, \ldots, \frac{k}{2}\right\}
$$

with

$$
\left\{\begin{array}{r}
f\left(e_{i}\right)+f\left(e_{j}\right)+f\left(e_{k}\right) \leq k \\
\left|f\left(e_{i}\right)-f\left(e_{j}\right)\right| \leq f\left(e_{k}\right) \leq f\left(e_{i}\right)+f\left(e_{j}\right), \\
f\left(e_{i}\right)+f\left(e_{j}\right)+f\left(e_{k}\right) \in \mathbf{Z},
\end{array}\right.
$$

for any three edges $e_{i}, e_{j}, e_{k}$ meeting in a vertex. The Verlinde space $V_{k}$ is the C-vector space with basis $b_{k}(\gamma)$; it has the same dimension as $S_{k}$.

The graph $\gamma$ corresponds to a pants decomposition of a genus two Riemann surface. Each vertex is a pair of pants, homeomorphic to $\mathbf{P}^{1}$ minus 3 points, the edges correspond to these points, which in turn correspond to 'vanishing cycles' on the Riemann surface. The mapping class group has a projective representation on $V_{k}$. The Dehn twist in a vanishing cycle corresponding to an edge $e$ of $\gamma$ acts, up 
to a scalar multiple and conjugation, by the diagonal matrix with entries (cf. [K2], p. 217 ; p. $214,(2-1))$

$$
\exp (-2 \pi i f(e)(f(e)+1) /(k+2)) \quad\left(f \in b_{k}(\gamma)\right) .
$$

Using this recipe we find the same results as before (see Lemmas 4.5.4 and 4.5.6):

4.5.8. Lemma. With the notation as above:

1. The eigenvalues of the Dehn twist in a non-separating cycle on $V_{k}$ are $\exp (-2 \pi i v(v+1) /(k+2))$ with multiplicity $(k-2 v+1)(2 v+1)$ where $v \in$ $\left\{0, \frac{1}{2}, 1, \ldots, \frac{k}{2}\right\}$.

2. The eigenvalues of a Dehn twist in a separating cycle on the space $V_{k}$ are $\exp (-2 \pi i l(l+1) /(k+2))$ with multiplicity $(k-2 l+1)^{2}$ where $l$ is an integer with $0 \leq l \leq k / 2$.

Proof. For the first statement consider the graph $\gamma$ with two vertices joined by three edges $e_{1}, e_{2}, e_{3}$. The edge $e_{1}$ corresponds to a non-separating cycle and one must determine $f\left(e_{1}\right)$ for all $f \in b_{k}(\gamma)$.

For the second case the recipe is not explicitly given in [K2], but is similar to the one used in the first case and gives a result that agrees with Lemma 4.5.6. One now takes $\delta$ to be the graph with two vertices $v_{1}, v_{2}$ and three edges $e_{1}, e_{2}, e_{3}$ such that the beginning and end of $e_{i}$ is $v_{i}$ and where $e_{3}$ connects $v_{1}$ and $v_{2}$. Thus the edge $e_{3}$ corresponds to a separating vanishing cycle and one has to determine $f\left(e_{3}\right)$ for all $f \in b_{k}(\delta)$. The computations are elementary and we leave them to the reader.

4.5.9. The cases $k=1,2$. The case $k=1$ is particularly easy since the operators $\Omega_{i j}$ are homogeneous of degree two and thus act as zero on $S_{1}$. The monodromy representation then factors over the projective representation of $A(G)$.

In the case $k=2$, the space $S_{k}$ is a direct sum of 10 distinct, one-dimensional $G$-representations (this is easy to verify, see also $[\mathrm{vG}]$ ). On each eigenspace, any $\sigma \tilde{\rho}_{s}\left(\Omega_{i j}\right)$ acts as scalar multiplication by an integer. A (local) basis of flat sections of $S_{2} \otimes \mathcal{P}$ is then given by 10 functions of the type $\left(\prod\left(z_{i}-z_{j}\right)^{r_{i j m}}\right) Q_{m}$ with $r_{i j m} \in \mathbf{Q}$ and $Q_{m} \in S_{2}$ is a basis of the eigenspace. These eigenspaces are permuted by the projective representation of $A(G)$.

\section{REFERENCES}

[BM] J.-L. Brylinski and D. McLaughlin, Holomorphic Quantization and Unitary Representations of the Teichmüller group, in: Lie Theory and Geometry (ed. J.-L. Brylinski et al.), Birkhäuser PM 123 (1994). MR 97d:32041

[D] P. Deligne, Equations Différentielles à Points Singuliers Réguliers, Springer LNM 163. MR 54:5232

[DO] I. Dolgachev and D. Ortland, Point sets in projective spaces and theta functions, Astérisque 165 (1988). MR 90i:14009

[DR] U. V. Desale and S. Ramanan, Classification of vector bundles of rank 2 on hyperelliptic curves, Invent. Math. 38 (1976) 161-185. MR 55:2906

[FH] W. Fulton and J. Harris, Representation Theory, GTM 129, Springer (1991). MR 93a:20069

[vG] B. van Geemen, Schottky-Jung relations and vector bundles on hyperelliptic curves, Math. Ann. 281 (1988) 431-449. MR 89f: 14046

[vGP] B. van Geemen and E. Previato, On the Hitchin system, Duke Math. J. 85 (1996) 659-683. CMP 97:05

[Ha] J. Harris, Algebraic Geometry, GTM 133, Springer (1992). MR 93j:14001

[Hi] N. Hitchin, Flat Connections and Geometric Quantization, Comm. Math. Phys. 131 (1990), 347-380. MR 91g:32022 
[Ka] C. Kassel, Quantum Groups, GTM 155, Springer (1995). MR 96e:17041

[K1] T. Kohno, Linear representations of braid groups and classical Yang-Baxter equations, in: Braids, Contemp. Math. 78, (editors: J.S. Birman and A. Libgober), AMS (1988). MR 90h:20056

[K2] T. Kohno, Topological invariants for 3-manifolds using representations of mapping class groups I, Topology, 31 (1992) 203-230. MR 94c:57031

[M] D. Mumford, Tata Lectures on Theta II, Progress in Math. 43, Birkhäuser. MR 86b:14017

[MS] G. Moore and N. Seiberg, Classical and quantum conformal field theory, Comm. Math. Phys. 123 (1989) 177-254. MR 90e:81216

[NR] M. S. Narasimhan and S. Ramanan, Moduli of vector bundles on a compact Riemann surface, Ann. of Math. 89 (1969) 19-51. MR 39:3518

[Si $\quad$ C. Simpson, Higgs bundles and local systems, Publications Mathematiques I.H.E.S. 75 (1992), 5-95. MR 94d:32027

[W] G. E. Welters, Polarized abelian varieties and the heat equations, Comp. Math. 49 (1983) 173-194. MR 85f: 14045

[Wr] G. Wright, The Reshetikhin-Turaev representation of the mapping class group, Journal of Knot Th. and Its Ram. 3 (1994) 547-574. MR 95k:57028

Dipartimento di Matematica, Università di Torino, Via Carlo Alberto 10, 10123 TORINO, ITALY

E-mail address: geemen@dm.unito.it

Department of Mathematics, Princeton University, Fine Hall - Washington Road, Princeton, New Jersey 08544-1000

E-mail address: dejong@math.Princeton.EDU 